\title{
A Low Temperature Expansion for Classical $N$-Vector Models. I. A Renormalization Group Flow
}

\section{Tadeusz Balaban}

Department of Mathematics, Boston University, Boston, MA 02215, USA

Received: 4 June 1993/in revised form: 28 March 1994

\begin{abstract}
A class of low temperature lattice classical spin models with a symmetry group $O(N)$ is considered, including the classical Heisenberg model. In this paper a renormalization group approach in a small field approximation is formulated and studied, with a goal to prove the so-called "spin wave picture" displaying massless behavior of the models.
\end{abstract}

\section{A Renormalization Group Flow}

\section{Introduction}

We consider a model for classical $N$-vector variables $\phi$ defined on a lattice $\mathbf{Z}^{d}$, $\phi(x) \in \mathbf{R}^{N}$ for $x \in \mathbf{Z}^{d}$. It is a lattice " $\lambda|\phi|^{4}$ " type field theory. To determine its thermodynamic properties we apply the usual thermodynamic procedure of taking limits of the corresponding finite volume models. We define them on tori $T=\{x \in$ $\mathbf{Z}^{d}$ : $\left.-L_{\mu} \leqq x_{\mu}<L_{\mu}, \mu=1, \ldots, d\right\}$ with periodic boundary conditions. A probability measure connected with a torus $T$ is defined by

$$
d \mu(\phi)=\rho(\phi) d \phi, \rho(\phi)=\exp [-\beta A(\phi)-E],
$$

where $d \phi$ is the Lebesgue measure on the space of all configurations $\phi$ defined on the torus $T, \beta>0$ is a parameter proportional to the inverse temperature $\beta=\frac{1}{k T}$, $E$ is a normalization constant, $E=\log Z, Z=\int d \phi \exp [-\beta A(\phi)]$. The action $A(\phi)$ is defined by

$$
\begin{aligned}
A(\phi)= & \frac{1}{2} \sum_{\left\langle x, x^{\prime}\right\rangle \subset T}\left|\phi(x)-\phi\left(x^{\prime}\right)\right|^{2}+\frac{\lambda}{8} \sum_{x \in T}|\phi(x)|^{4}-\frac{\mu}{2} \sum_{x \in T}|\phi(x)|^{2} \\
& -\sum_{x \in T} h \cdot \phi(x)=\frac{1}{2}\|\partial \phi\|^{2}+\frac{\lambda}{8}\left\||\phi|^{2}\right\|^{2}-\frac{\mu}{2}\|\phi\|^{2}-\langle h, \phi\rangle,
\end{aligned}
$$


where $\lambda>0, \mu>0, h \in \mathbf{R}^{N}$. The effective potential for this action is given by

$$
V(\phi)=\frac{\lambda}{8}|\phi|^{4}-\frac{\mu}{2}|\phi|^{2}-h \cdot \phi=\frac{\lambda}{8}\left(|\phi|^{2}-\frac{2 \mu}{\lambda}\right)^{2}-v h_{0} \cdot \phi-\frac{\mu^{2}}{2 \lambda},
$$

where $h=v h_{0}, v \geqq 0,\left|h_{0}\right|=1$. For $v>0$ it has exactly one minimum at a vector proportional to $h_{0}$. It is convenient to normalize the potential in such a way that this minimum is exactly equal to $h_{0}$. This can be achieved simply by scaling the variables $\phi$. Denoting the rescaled coefficients in the same way we obtain the effective potential of the form

$$
V(\phi)=\frac{\lambda}{8}\left(|\phi|^{2}-\rho\right)^{2}-v h \cdot \phi-\frac{\lambda}{8} \rho^{2},
$$

where $h_{0}$ is denoted by $h$. This potential has a minimum at $\phi=h$ if $\rho=1-\frac{2 v}{\lambda}$. Assuming this we can finally write the action in the following form:

$$
A(\phi)=\frac{1}{2}\|\partial \phi\|^{2}+\frac{\lambda}{8}\left\||\phi|^{2}-1\right\|^{2}+\frac{v}{2}\|\phi-h\|^{2},
$$

if we rescale properly the parameter $\beta$ also. The constant arising from these transformations is included in $E$. We consider this model for $\beta$ sufficiently large, $\lambda$ not too small, for simplicity we assume that $\lambda \geqq 1, v>0$ and $h$ in the unit sphere of $\mathbf{R}^{N}, h \in S^{N-1}$. We want to include the case $\lambda=+\infty$, because all the constructions and proofs in this paper are uniform for $\lambda \in[1,+\infty]$. This case is understood as the limit

$$
\begin{aligned}
& \lim _{\lambda \rightarrow+\infty} \exp \left[-\beta A(\phi)-E_{\lambda}\right] \\
& \quad=\exp \left[-\beta\left(\frac{1}{2}\|\partial \phi\|^{2}+\frac{v}{2}\|\phi-h\|^{2}\right)-E_{\infty}\right] \prod_{x \in T} \delta\left(|\phi(x)|^{2}-1\right),
\end{aligned}
$$

which is a classical Heisenberg model, or its $\mathrm{N}$-component generalization.

Let us make a comment on other possible parametrizations of the model. Rescaling $\phi$ by $\beta^{-\frac{1}{2}}$ we obtain the density $\rho(\phi)=\exp [-A(\phi)-E]$ with the action

$$
\begin{aligned}
A(\phi)= & \frac{1}{2}\|\partial \phi\|^{2}+\frac{\lambda \beta^{-1}}{8}\left\||\phi|^{2}-\beta\right\|^{2}+\frac{v}{2}\left\|\phi-\beta^{\frac{1}{2}} h\right\|^{2} \\
= & \frac{1}{2}\|\partial \phi\|^{2}+\frac{\lambda \beta^{-1}}{8}\left\||\phi|^{2}\right\|^{2}-\frac{1}{2}\left(\frac{1}{2} \lambda-v\right)\|\phi\|^{2}-v \beta^{\frac{1}{2}}\langle\phi, h\rangle \\
& +\left(\frac{\lambda \beta}{8}+\frac{v \beta}{2}\right)|T| .
\end{aligned}
$$

For $\lambda \approx 1, v$ small, this is an immediate $N$-component generalization of the lattice version of the model considered in [4]. All the above representations are connected by simple scaling transformations, so they have exactly the same properties, but there are differences on a technical level, in particular renormalization procedures will have different forms. We have found the form (1.5) most convenient to work with. 
The action (1.5) has some important symmetries. The first two terms are invariant with respect to transformations of the orthogonal group $O(N)$. The third term breaks this symmetry, it is invariant with respect to the subgroup of $O(N)$ leaving the vector $h$ invariant. This can be formulated also as an invariance with respect to transformations of $O(N)$ acting simultaneously on both variables $\phi, h$. The action is also invariant with respect to Euclidean transformations of the lattice $T$. Let us write the action $A(\phi)$ more explicitly as $A(\phi ; h, \lambda, v)$. The symmetries can be formulated as the equalities

$$
\begin{gathered}
A(R \phi ; R h, \lambda, v)=A(\phi ; h, \lambda, v) \text { for all } R \in O(N), \\
A(r \phi ; h, \lambda, v)=A(\phi ; h, \lambda, v) \text { for all Euclidean transformations } \\
r \text { of the lattice } T, \text { where }(r \phi)(x)=\phi(r x), x \in T .
\end{gathered}
$$

In particular the Euclidean symmetries include all translations of the torus $T$.

Let us recall the well-known heuristic picture for this model, the so-called "spin wave picture," e.g. see $[7,9]$. According to it the model has exactly one state in a thermodynamic limit if $v>0$. Denote the state, or rather the corresponding expectation value, by $\langle\cdot\rangle_{v}$. It is determined by the parameters $\beta, h, \lambda, \nu$, we have written explicitly the dependence on $v$ only. This state has a non-zero magnetization, i.e. a non-zero expectation value $\langle\phi(x)\rangle_{v}$. By the symmetries (1.7), which imply the corresponding symmetries of the finite volume states, and these are preserved in the thermodynamic limit, this expectation value must be a constant vector proportional to $h$. Thus we can write $\langle\phi(x)\rangle_{v}=m_{v} h$, and the statement is that for $\beta$ sufficiently large $m_{v}>0$. The two-point truncated correlation function $\left\langle\phi(x) \phi\left(x^{\prime}\right)\right\rangle_{v}^{T}=\left\langle\phi(x) \phi\left(x^{\prime}\right)\right\rangle_{v}-\langle\phi(x)\rangle_{v}\left\langle\phi\left(x^{\prime}\right)\right\rangle_{v}$ and higher-point truncated correlation functions have an exponential decay with a decay rate proportional to $\sqrt{1}$. These states have a limit as $v \rightarrow 0+, \lim _{v \rightarrow 0+}\langle\cdot\rangle_{v}=\langle\cdot\rangle_{0+}$. For $\beta$ sufficiently large the limit has a non-zero spontaneous magnetization $\langle\phi(x)\rangle_{0+}=m_{0+} h$, $m_{0+}=\lim _{v \rightarrow 0+} m_{v}>0$, and the truncated correlation functions have a power law decay. The decay is quite complicated and depends on components of correlation functions. For transversal components of the two-point function, i.e. for components in directions orthogonal to the vector $h$, the decay is as in asymptotically free massless theories, i.e. it is given by the operator $(-\Delta)^{-1}$. The above picture holds for $d \geqq 3, N \geqq 2$, and can be obtained from a properly constructed perturbative expansion, e.g. see [9].

There is quite a number of rigorous mathematical results concerning the above and related models. We do not intend to make a survey of those results, but let us mention a basic paper [5] in which some fundamental aspects of the above picture have been proved, like existence of the non-zero magnetization, domination of the two-point correlation function by the free massless Green's function, etc. These and the other results have been obtained by very elegant and simple methods, like infrared bounds, chessboard bounds, correlation inequalities, etc. We develop here a different approach based on expansion methods, so we do not go into any further details of the above mentioned results. In this series of papers we will eventually prove all the statements of the heuristic picture, but our fundamental goal is to construct a convergent low-temperature expansion for the considered model, an 
analog of such expansions for the Ising model, or for this model with $N=1$ constructed in [4]. A reason for this is that our interests are not in this particular model, but in corresponding models with various disorders, or with stochastic dynamics. It turns out that to understand these more complicated models it is crucial to get precise results on this basic and simplest one, underlying all the others. Moreover it turns out that it is not enough to have even the most precise results, but it is important to have a sufficiently flexible and robust expansion method. This should be clear from the paper [4], in which the Ising model plays the role of the basic underlying model, and where it is crucial to have the lowtemperature Peierls expansion for it, not just its properties. This is why the emphasis in these papers is almost entirely on a construction of such an expansion method. It should be clear also that in our case it must be a multi-scale expansion, because of massless modes, or slow decay properties of correlation functions. We construct it using renormalization group ideas and techniques, in particular the ideas and techniques developed for lattice scalar and gauge field models in [1a, $b$, $2 \mathrm{a}-\mathrm{e}]$.

An important ingredient of any renormalization group program is to establish how scaling transformations of a space act on various quantities of a considered model. Let us describe these scaling transformations in our model. We scale the unit lattice $T$ by a positive number $\varepsilon$, so we obtain the $\varepsilon$-lattice $T_{\varepsilon}=\{\varepsilon x: x \in T\}$. The effective potential for the action (1.5) is given by

$$
V(\phi)=V(\phi ; h, \lambda, v)=\frac{\lambda}{8}\left(|\phi|^{2}-1\right)^{2}+\frac{v}{2}|\phi-h|^{2} .
$$

It has a minimum at $\phi=h$, and for $v=0$ a set of minima is the unit sphere $|\phi|=1$. We want to preserve this property by the scaling transformations, so the variable $\phi$ does not scale, or rather it scales with the trivial factor $\varepsilon^{0}=1$, and we have $\phi(\varepsilon x)=\phi(x)$. Actually we should distinguish between the two configurations defined on the lattices with different scales, but because of this scaling property we always use the same notation for configurations defined on rescaled lattices. Using this property we have

$$
\begin{aligned}
\beta A(\phi ; h, \lambda, v)= & \beta \varepsilon^{2-d}\left[\frac{1}{2} \sum_{\left\langle x, x^{\prime}\right\rangle \subset T_{\varepsilon}} \varepsilon^{d}\left|\left(\partial^{\varepsilon} \phi\right)\left(\left\langle x, x^{\prime}\right\rangle\right)\right|^{2}\right. \\
& \left.+\frac{\lambda \varepsilon^{-2}}{8} \sum_{x \in T_{\varepsilon}} \varepsilon^{d}\left(|\phi(x)|^{2}-1\right)^{2}+\frac{v \varepsilon^{-2}}{2} \sum_{x \in T_{\varepsilon}} \varepsilon^{d}|\phi(x)-h|^{2}\right] \\
= & \beta \varepsilon^{2-d} A^{\varepsilon}\left(\phi ; h, \lambda \varepsilon^{-2}, v \varepsilon^{-2}\right)=\beta^{\varepsilon} A^{\varepsilon}\left(\phi ; h, \lambda^{\varepsilon}, v^{\varepsilon}\right),
\end{aligned}
$$

where $A^{\varepsilon}$ denotes the action with all the expressions, like derivatives, norms, etc. defined on the $\varepsilon$-lattice, and $\beta^{\varepsilon}, \lambda^{\varepsilon}, v^{\varepsilon}$ are the corresponding coefficients calculated for this scale. From (1.9) we obtain the scaling laws for the coefficients

$$
\beta^{\varepsilon}=\beta \varepsilon^{2-d}, \quad \lambda^{\varepsilon}=\lambda \varepsilon^{-2}, \quad v^{\varepsilon}=v \varepsilon^{-2} .
$$

These are the basic, canonical scalings. Renormalizations connected with the renormalization group approach introduce some additional changes of the coefficients, but for $d>2$ the canonical scalings dominate an asymptotic behavior as 
$\varepsilon \rightarrow 0$. In particular they decrease the temperature, strengthen an effect of a barrier well around the sphere $|\phi|=1$ by increasing $\lambda$, and they increase the external field $v$. Our approach to the low temperature problem for this model is based on these scaling properties.

Let us describe now some basic features of the renormalization group approach we apply here. We will be very brief because it has been described already several times, so we refer the reader to the previous papers for details, in particular to $[1 \mathrm{a}, 2 \mathrm{a}, \mathrm{c}]$. We apply a sequence of renormalization transformations with linear averaging operations and Gaussian densities. For future references let us recall the most important definitions. A renormalization transformation applied to a density $\rho(\phi, \beta)$, depending on the parameter $\beta$, is defined by the formulas

$$
\begin{aligned}
(T \rho)(\psi) & =\int d \phi t(\psi, \phi ; \beta, a) \rho(\phi, \beta), \\
t(\psi, \phi ; \beta, a) & =\prod_{y \in T_{t:}^{(1)}} t(y ; \psi, \phi ; \beta, a), \\
t(y ; \psi, \phi ; \beta, a) & =\exp \left[-\frac{1}{2} \beta a L^{d-2}|\psi(y)-(Q \phi)(y)|^{2}+\frac{N}{2} \log \frac{\beta a L^{d-2}}{2 \pi}\right], \\
(Q \phi)(y) & =\sum_{x \in B(v)} L^{-d} \phi(x),
\end{aligned}
$$

where $B(y)$ denotes the $L$-block with center at $y \in T_{L}^{(1)}, T_{L}^{(1)}$ denotes the new $L$-lattice of centers of the blocks, and $\psi$ is a new spin configuration defined on the new lattice. Let us make a few comments on this definition. At first, it has the usual normalization property: $\int d \psi(T \rho)(\psi)=\int d \phi \rho(\phi)$. Second, we take a $\approx 1$, e.g. $\frac{1}{2}<a<\frac{3}{2}$; later we will specify this constant more precisely. The definition depends also explicitly on the parameter $\beta$. In a next step this parameter is rescaled as in (1.10) and renormalized, so the next renormalization transformation depends on a different $\beta$. Thus we do not iterate simply the above transformation, but we take a product of different transformations of the form (1.11) determined by a sequence of the so-called running, or partially renormalized constants $\beta_{k}$. We renormalize also the new spin variable $\psi$ by applying a properly chosen scaling transformation. To indicate these changes we introduce the subscript $k$ denoting new constants and variables after $k$ transformations, starting with $k=0$ for those in (1.1), (1.5). The renormalization transformations (1.11) are followed by scaling transformations which rescale L-lattices of new spin variables into unit lattices, for example the lattice $T_{L}^{(1)}$ in (1.11) into the unit lattice $T_{1}^{(1)}$. They do not change spin configurations, as has been discussed above, and they are denoted by $S$. Actually we write the transformations $S, T$ with superscripts indicating to what densities they are applied, for example we denote the density given by (1.1), (1.5) by $\rho_{0}$, and the transformations applied to it have the superscript " 0 ." Thus applying $k$ renormalization and scaling transformations yields a density $\rho_{k}$ for which

$$
\rho_{k}=S^{(k-1)} T^{(k-1)} \rho_{k-1}=\prod_{j=1}^{k} S^{(k-j)} T^{(k-j)} \rho_{0}=(S T)_{k} \rho_{0} .
$$

The basic goal is to give a sufficiently detailed and precise description of the densities $\rho_{k}$. 
To cover also a renormalization group analysis of correlation functions we consider a density of a generating functional for the correlation functions, which means simply that we add the linear function $\langle g, \phi\rangle$ to the exponent in (1.1), where $g$ is defined on $T$ and has values in $\mathbf{C}^{N}$. We denote this density by $\rho_{0}$, so $\rho_{k}$ denotes a density of the generating functional after the $k$ transformations.

To understand better various issues connected with an inductive description of the densities $\rho_{k}$ we discuss briefly some basic aspects of the first renormalization transformation applied to $\rho_{0}$. It is defined by the integral

$$
\begin{aligned}
\left(T^{(0)} \rho_{0}\right)(\psi)= & \int d \phi \exp \left[-\beta_{0}\left\{\frac{1}{2} a L^{-2}\|\psi-Q \phi\|_{L}^{2}+\frac{1}{2}\|\partial \phi\|^{2}\right.\right. \\
& \left.\left.+\frac{\lambda_{0}}{8}\left\||\phi|^{2}-1\right\|^{2}+\frac{v_{0}}{2}\|\phi-h\|^{2}\right\}+\langle g, \phi\rangle-E_{0}^{\prime}\right],
\end{aligned}
$$

where the normalization constant of the renormalization transformation is included in the constant $E_{0}^{\prime}$. Consider the underintegral expression in (1.13) and define

$$
\begin{aligned}
A_{1}\left(\psi, \phi ; h, a L^{-2}, \lambda_{0}, v_{0}\right)= & \frac{1}{2} a L^{-2}\|\psi-Q \phi\|^{2}+\frac{1}{2}\|\partial \phi\|^{2}+\frac{i_{0}}{8}\left\|\left.\phi\right|^{2}-1\right\|^{2} \\
& +\frac{v_{0}}{2}\|\phi-h\|^{2} .
\end{aligned}
$$

We are interested in low temperature properties of the model, so $\beta_{0}$ may be arbitrarily large and it is natural to apply a saddle point method to calculate the integral (1.13). This method requires finding all critical points of the function (1.14), which is multiplied by $\beta_{0}$ in (1.13). The problem of finding the critical points on the whole space of configurations $\phi$ is a highly singular one; it is easy to construct examples of configurations $\psi$ with many critical points, in fact with quite singular sets of critical points, and with a discontinuous dependence on $\psi$. Also, expanding the function (1.14) around some of the critical points we obtain functions with no good positivity properties, so the resulting integrals cannot be treated by any known method. To avoid all these problems we have to introduce some restrictions on configurations $\psi, \phi$. These restrictions are naturally connected with positive terms in the effective action (1.14), for example they may be defined by

$$
\begin{aligned}
|\psi(y)-(Q \phi)(y)| & <\beta_{0}^{-\frac{1}{2}} p\left(\beta_{0}\right),|(\partial \phi)(b)|<\beta_{0}^{-\frac{1}{2}} p\left(\beta_{0}\right), \\
\left.|| \phi(x)\right|^{2}-1 \mid & <\lambda_{0}^{-\frac{1}{2}} \beta_{0}^{-\frac{1}{2}} p\left(\beta_{0}\right),|\phi(x)-h|<v_{0}^{-\frac{1}{2}} \beta_{0}^{-\frac{1}{2}} p\left(\beta_{0}\right), p\left(\beta_{0}\right) \\
& =\left(A_{0}+\log \beta_{0}\right)_{0},
\end{aligned}
$$

where $A_{0}$ is a positive constant and $p_{0}$ is an even integer. These constants have to be chosen to satisfy various conditions appearing later in this and subsequent papers. The restrictions (1.15) are introduced in the following way. We decompose the space of all configurations $\phi$ into subsets, each subset being characterized by the condition that at each point or bond of a corresponding lattice either an 
inequality in (1.15), or a complementary inequality holds. For a given subset denote by $X$ the set of points and bonds at which the complementary inequalities hold. This is, rought speaking, "a large field domain" and the part $\exp \left[-\beta_{0} A_{1}\right]$ of the density $\rho_{0}$ restricted to this domain can be bounded by $\exp \left[-\frac{1}{8} p^{2}\left(\beta_{0}\right)|X|\right]$. The integral of the density restricted to the domain can be bounded also by such a factor with the constant $\frac{1}{8}$ replaced by some other positive number. This factor is sufficiently small to control the combinatorics of the decomposition. On the complement of the set $X$ the inequalities (1.15) hold, so it is "a small field domain," and the integral restricted to this domain can be calculated by the saddle point method indicated above. The action $A_{1}$ restricted to the domain has exactly one critical point, and its expansion around the point has sufficiently good properties, so that the integral can be analyzed by a simplified version of a cluster expansion. In the first three papers we discuss problems connected with the small field domains. For simplicity and clarity we discuss mainly the case when a whole lattice in each step is the small field domain. Considering a general case in a final paper will be quite simple, it will demand only minor modifications of the analysis in this special case. Thus we consider here the sequence of effective densities $\rho_{k}$ restricted to properly defined small field spaces and determined by the renormalization group transformations restricted also to some small field spaces. Thus instead of the densities given by the formula (1.12) we consider here the small field densities denoted also by $\rho_{k}$ and given by the formula

$$
\rho_{k}=\chi_{k} S^{(k-1)} T^{(k-1)} \chi^{(k-1)} \cdots S^{(0)} T^{(0)} \chi^{(0)} \rho_{0},
$$

where the characteristic function $\chi_{k}, \chi^{(j)}$ yield restrictions to proper small field spaces. They will be defined precisely later on, but the idea is that they are essentially equivalent to restrictions of the form (1.15), only for technical reasons they are formulated in a more elaborate way.

The basic goal of this paper is to give a precise description of the densities $\rho_{k}$ and to prove some of their properties and bounds. This description will be given in the next section, together with a formulation of a main theorem of this and two subsequent papers. In the remaining two sections of this paper we analyze the densities, or rather corresponding effective actions, and we prove proper bounds for them. The next paper deals with the problem of critical points for generalizations of the function (1.14). We prove there all technical results about solutions of the corresponding variational problems needed in the rest of this work. In the third paper we finish the inductive proof of the main theorem. In the last paper we consider the general case including contributions from the large field domains and analyzing their renormalization and bounds. We hope that the papers are selfcontained, but in case some doubts arise in connection with notations used here we refer the reader to $[2 \mathrm{a}, \mathrm{c}]$ for more detailed definitions.

\section{An Inductive Description of the Effective Densities and a Formulation of the Main Theorem}

A fundamental role in the inductive description is played by critical points of effective actions which are simple generalizations of the action (1.14). These critical 
points turn out to be minima of the corresponding actions, so we consider the appropriate variational problems. Let us start with definitions. We rescale the lattice $T$ to the scale $\eta=L^{-k}$, so we get the lattice $T_{\eta}$. Spin configurations $\phi$ are defined on $T_{\eta}$, and new spin configurations $\psi$ are defined on the lattice $T_{1}^{(k)}$, which is a unit lattice of centers of $k$-blocks, i.e. blocks containing $L^{k d}$ sites of $T_{\eta}$. We define

$$
\begin{aligned}
A_{k}(\psi, \phi ; h, a, \lambda, v)= & \frac{a}{2}\left\|\psi-Q_{k} \phi\right\|_{1}^{2}+\frac{1}{2}\left\|\partial^{n} \phi\right\|^{2} \\
& +\frac{\lambda}{8}\left\||\phi|^{2}-1\right\|^{2}+\frac{v}{2}\|\phi-h\|^{2}, \quad \eta=L^{-k},
\end{aligned}
$$

where the norms are defined on the $\eta$-lattice, except the first one which is defined on the unit lattice. We want to solve the problem of finding

$$
\inf _{\phi} A_{k}(\psi, \phi ; h, a, \lambda, v) \text {. }
$$

This problem is analyzed in detail in the next paper, where we prove that in a proper small field domain there exists exactly one critical point of the function $A_{k}$. This point is a minimum of the function, so it is a solution of the problem (2.2). We denote this minimal configuration by $\phi_{k}$. It is an analytic function of $\psi, h, a, \lambda, v$ on corresponding domains, and it has some regularity properties as a function on the lattice $T_{\eta}$. These properties will be described in detail later on. Let us mention that the above problem and properties of solutions are most naturally described on the $\eta$-scale, but they can be rescaled and formulated on any other scale. The rescaling has been explained in the Introduction, we change properly scales of the lattices only; spin configurations are unchanged. For example consider a minimal configuration $\phi_{j}$ for some $j<k$. It is determined naturally by the corresponding function $A_{j}$ given by (2.1) on the lattice $T_{\xi}, \xi=L^{-j}$, as the solution of the problem (2.2). We have

$$
A_{j}(\psi, \phi ; h, a, \lambda, v)=\left(L^{j} \eta\right)^{-(d-2)} A_{j}^{\eta}\left(\psi, \phi ; h, a\left(L^{j} \eta\right)^{-2}, \lambda\left(L^{j} \eta\right)^{-2}, v\left(L^{j} \eta\right)^{-2}\right),
$$

where the function on the left-hand side is defined for spin configurations $\psi, \phi$ and norms on the 1-lattice $T_{1}^{(j)}$, and the $\xi$-lattice $T_{\xi}$ correspondingly, and the function on the right-hand side is defined for the rescaled configurations $\psi, \phi$ and norms on the $L^{j} \eta$-lattice $T_{L^{\prime} \eta}^{(j)}$, and the $\eta$-lattice $T_{\eta}$ correspondingly. The above equality implies the following scaling law for the minimal configurations $\phi_{j}$ :

$$
\phi_{j}(x ; \psi, h, a, \lambda, v)=\phi_{j}\left(L^{j} \eta x ; \psi, h, a\left(L^{j} \eta\right)^{-2}, \lambda\left(L^{j} \eta\right)^{-2}, v\left(L^{j} \eta\right)^{-2}\right), \quad x \in T_{\xi},(2.4
$$

where the configuration $\psi$ is simply rescaled by the equality $\psi(y)=\psi\left(L^{j} \eta y\right)$, $y \in T_{1}^{(j)}$. A minimal configuration $\phi_{j}$ considered as a function of lattice points only is also rescaled in the same simple way.

A first inductive assumption is about a general form of the $k^{\text {th }}$ density $\rho_{k}$. We assume that

$$
\begin{aligned}
\rho_{k}\left(\psi_{k}\right) & =\chi_{k} \exp \left[\mathscr{A}_{k}+\mathscr{F}_{k}\left(\psi_{k}, h, g\right)\right], \\
\mathscr{A}_{k} & =-\beta_{k} A_{k}\left(\psi_{k}, \phi_{k} ; h, a_{k}, \lambda_{k}, v_{k}\right)+\mathscr{E}_{k}\left(\psi_{k}, h\right)-E_{k},
\end{aligned}
$$

where $\psi_{k}$ is a new spin configuration on the unit lattice $T_{1}^{(k)}$. The coefficients $\beta_{k}, a_{k}, \lambda_{k}, v_{k}$ are determined by a sequence of inductive renormalization equations, 
which will be discussed in the third paper. Now let us mention only that the leading asymptotic behavior of these coefficients is determined by the scaling (1.10), i.e. we have approximately

$$
\beta_{k} \approx \beta L^{k(d-2)}, \quad a_{k} \approx a \frac{1-L^{-2}}{1-L^{-2 k}}, \quad \lambda_{k} \approx \lambda L^{2 k}, \quad v_{k} \approx \nu L^{2 k} .
$$

The minimal configuration $\phi_{k}$ is determined by $\psi_{k}, h$ and the coefficients $a_{k}, \lambda_{k}, v_{k}$.

We shall describe successively inductive assumptions for the remaining expressions in the effective density $\rho_{k}$.

Let us begin with the term $\mathscr{E}_{k}$ in the effective $\mathscr{A}_{k}$. It demands a most detailed and careful description because the renormalization operations are determined by its properties. Before we begin this description we have to introduce another class of minimal configurations. They are solutions of variational problems which connect functions $\phi_{j}$ and $\phi_{k}$. The configurations $\phi_{j}$ are determined by the function (2.3) as solutions of the problem (2.2). If we compose the next $k-j$ renormalization transformations $T^{(j)}, \ldots, T^{(k-1)}$, we obtain a renormalization transformation with a Gaussian kernel determined by the averaging operation $Q_{k-j}$ and some constant $a_{k-j}$. A quadratic form of this kernel combined with the main term of the $\mathrm{j}^{\text {th }}$ effective action yield a function of a new spin variable $\psi_{j}$ of the $j^{\text {th }}$ action. We want to find a minimum of this function, i.e. to solve a variational problem,

$$
\inf _{\psi_{,}}\left\{\frac{1}{2} a_{k-j}\left\|\psi_{k}-Q_{k-j} \psi_{j}\right\|_{1}^{2}+A_{j}^{\eta}\left(\psi_{j}, \phi_{j} ; h, a_{j}\left(L^{j} \eta\right)^{-2}, \lambda_{j}\left(L^{j} \eta\right)^{-2}, v_{j}\left(L^{j} \eta\right)^{-2}\right)\right\} .
$$

The function $A_{j}$ has been rescaled to the $\eta$-lattice by (2.3), and the scaling factor $\left(L^{j} \eta\right)^{-(d-2)}$ has been incorporated into the constant $\beta_{j}$. We disregard at this moment changes connected with the renormalization operations, we want to capture only a leading effect of the renormalization transformations. The above problem is analyzed in detail in the next paper, where we prove that in a proper small field region there exists exactly one critical point of the function in (2.6), which is a minimum of this function, so it is a solution of the problem (2.6). Denote this solution by $\psi_{k}^{(j)}$. It is a function of $\psi_{k}, h$ and the coefficients determining the function in (2.6), so we may write more precisely $\psi_{k}^{(j)}\left(\psi_{k} ; h, a_{k-j}, a_{j}\left(L^{j} \eta\right)^{-2}, \lambda_{j}\left(L^{j} \eta\right)^{-2}, v_{j}\left(L^{j} \eta\right)^{-2}\right)$. It is a configuration defined on the lattice $T_{L^{\prime} \eta}^{(j)}$, and it has various analyticity and regularity properties described in that paper. The most important property is its relation to the functions $\phi_{k}$. It follows from the identity

$$
\begin{aligned}
(2.6)= & \inf _{\psi_{j}} \inf _{\phi}\left\{\frac{1}{2} a_{k-j}\left\|\psi_{k}-Q_{k-j} \psi_{j}\right\|_{1}^{2}+\frac{1}{2} a_{j}\left(L^{j} \eta\right)^{-2}\left\|\psi_{j}-Q_{j} \phi\right\|_{L^{\prime} \eta}^{2}\right. \\
& \left.+A_{0}^{\eta}\left(\phi ; h, \lambda_{j}\left(L^{j} \eta\right)^{-2}, v_{j}\left(L^{j} \eta\right)^{-2}\right)\right\}=\inf _{\phi}\left\{\frac{1}{2} \frac{a_{k-j} a_{j}}{a_{j}+a_{k-j}\left(L^{j} \eta\right)^{2}}\left\|\psi_{k}-Q_{k} \phi\right\|_{1}^{2}\right. \\
& \left.+A_{0}^{\eta}\left(\phi ; h, \lambda_{j}\left(L^{j} \eta\right)^{-2}, v_{j}\left(L^{j} \eta\right)^{-2}\right)\right\} \\
= & \inf _{\phi} A_{k}\left(\psi_{k}, \phi ; h, \frac{a_{k-j} a_{j}}{a_{j}+a_{k-j}\left(L^{j} \eta\right)^{2}}, \lambda_{j}\left(L^{j} \eta\right)^{-2}, v_{j}\left(L^{j} \eta\right)^{-2}\right)
\end{aligned}
$$


This identity means that if we substitute the solution of the problem (2.6) into the function $\phi_{j}$, we obtain a solution of the last problem above, i.e. a function $\phi_{k}$. The composition property $\phi_{j}{ }^{\circ} \psi_{k}^{(j)}=\phi_{k}$ can be written precisely in terms of the coefficients of the above problems as follows:

$$
\begin{gathered}
\phi_{j}\left(\psi_{k}^{(j)}\left(\psi_{k} ; h, a_{k-j}, a_{j}\left(L^{j} \eta\right)^{-2}, \lambda_{j}\left(L^{j} \eta\right)^{-2}, v_{j}\left(L^{j} \eta\right)^{-2}\right) ; h, a_{j}\left(L^{j} \eta\right)^{-2}, \lambda_{j}\left(L^{j} \eta\right)^{-2}, v_{j}\left(L^{j} \eta\right)^{-2}\right) \\
=\phi_{k}\left(\psi_{k} ; h, \frac{a_{k-j} a_{j}}{a_{j}+a_{k-j}\left(L^{j} \eta\right)^{2}}, \lambda_{j}\left(L^{j} \eta\right)^{-2}, v_{j}\left(L^{j} \eta\right)^{-2}\right) .
\end{gathered}
$$

In the above definitions and identities we have determined all the functions in terms of the coefficients of the $\mathrm{j}^{\mathrm{th}}$ action $A_{j}$ and the constant $a_{k-j}$, in particular the last configuration $\phi_{k}$ in (2.8) is such a function. In our inductive description of the effecitve action $\mathscr{E}_{k}\left(\psi_{k}, h\right)$ we assume that it depends on $\psi_{k}$ through the functions $\psi_{k}^{(j)}$, and we need a different interpretation of the above formulas. We require that the identities (2.7) yield always the main part of the effective action after $k$ steps, i.e. the action $A_{k}\left(\psi, \phi_{k} ; h, a_{k}, \lambda_{k}, v_{k}\right)$, and the identities (2.8) yield the corresponding minimal configuration $\phi_{k}\left(\psi_{k} ; h, a_{k}, \lambda_{k}, v_{k}\right)$, for all $j=1,2, \ldots, k-1$. This requirement determines the coefficients $\lambda_{j}, v_{j}$,

$$
\lambda_{j}=\lambda_{k}\left(L^{j} \eta\right)^{2}, \quad v_{j}=v_{k}\left(L^{j} \eta\right)^{2}
$$

and imposes the following sequence of equations:

$$
\frac{a_{k-j} a_{j}}{a_{j}+a_{k-j}\left(L^{j} \eta\right)^{2}}=a_{k}
$$

These equations can be solved with respect to the constant $a_{k}$, and the solutions are given by the formulas

$$
a_{j}=a_{k} \frac{1-L^{-2 k}}{1-L^{-2 j}}
$$

Let us remark that equalities (2.9), (2.11) would be satisfied for the actual renormalization group flow of the constants $\left\{\lambda_{j}, v_{j}, a_{j}\right\}$ if no renormalization operations were necessary and the constants were determined only by the scaling operations and compositions of the renormalization transformations. As the actual flow differs from such an ideal flow due to renormalization operations performed at each step, we interpret the equalities (2.9), (2.11) as definitions of this ideal flow matched to the constants $\lambda_{k}, v_{k}, a_{k}$ at the $\mathrm{k}^{\text {th }}$ step. Because of this dependence we should write the constants $\lambda_{j}, v_{j}, a_{j}$ with an additional index $k$, but for simplicity we keep the above notation. In almost the whole paper the index $k$ is fixed. Thus in the future when we write a configuration $\psi_{k}^{(j)}$ we understand by it the function

$$
\psi_{k}^{(j)}\left(\psi_{k} ; h, a_{k-j}, a_{j}\left(L^{j} \eta\right)^{-2}, \lambda_{k}, v_{k}\right)
$$

where $a_{k-j}, a_{j}$ are given by the formulas (2.11). Let us remark also that this function depends on $h$ through the product $v_{k} h$, but for simplicity of the formulas we have not written it explicitly, although we will use it in the future. Finally let us notice that the identities (2.7) allow us to write an explicit formula for the configuration $\psi_{k}^{(j)}$ in terms of $\phi_{k}$. Calculating the infimum with respect to $\psi_{j}$ for the function on the right-hand side of the first equality in (2.7) we obtain the following formula for the critical point:

$$
\psi_{j}=Q_{j} \phi+\frac{a_{k-j}\left(L^{j} \eta\right)^{2}}{a_{j}+a_{k-j}\left(L^{j} \eta\right)^{2}} Q_{k-j}^{*}\left(\psi_{k}-Q_{k} \phi\right)
$$


Substituting next $\phi_{k}$ instead of $\phi$, and using the equalities (2.10), (2.11), we obtain

$$
\begin{aligned}
\psi_{k}^{(j)} & =Q_{j} \phi_{k}+\frac{a_{k}}{a_{j}}\left(L^{j} \eta\right)^{2} Q_{k-j}^{*}\left(\psi_{k}-Q_{k} \phi_{k}\right) \\
& =Q_{j} \phi_{k}+\frac{1-L^{-2 j}}{1-L^{-2 k}}\left(L^{j} \eta\right)^{2} Q_{k-j}^{*}\left(\psi_{k}-Q_{k} \phi_{k}\right) .
\end{aligned}
$$

This formula is of fundamental importance for the future analysis of these functions, in particular for localization and regularity properties.

Now we can begin the inductive description of the function $\mathscr{E}_{k}\left(\psi_{k}, h\right)$. We assume that it is a sum of contributions coming from $k$ renormalization steps, and that each contribution can be represented as a sum of localized contributions on a corresponding lattice, i.e. it has basically the same form as in [2d]. We assume also that a $j^{\text {th }}$ contribution depends on $\psi_{k}$ through the function $\psi_{k}^{(j)}$. We write these assumptions as follows:

(H.2) there exist functions $\mathscr{E}^{(j)}\left(y ; \psi_{j}, v_{j} h\right)$, where $\psi_{j}$ is a spin variable on the lattice $T^{(j)}$, such that

$$
\begin{aligned}
\mathscr{E}_{k}\left(\psi_{k}, h\right) & =\sum_{j=1}^{k} \mathscr{E}^{(j)}\left(\psi_{k}^{(j)}\left(\psi_{k}, v_{k} h\right), v_{j} h\right), \\
\mathscr{E}^{(j)}\left(\psi_{j}, v_{j} h\right) & =\sum_{y \in T^{(j)}} \mathscr{E}^{(j)}\left(y ; \psi_{k}, v_{j} h\right) .
\end{aligned}
$$

Let us make a few remarks about the above assumption. The function $\mathscr{E}^{(j)}\left(\psi_{j}, v_{j} h\right)$ is constructed at the $j^{\text {th }}$ step, and $\psi_{j}$ is a new spin variable defined on the lattice $T_{1}^{(j)}$. These functions are renormalized, corresponding counterterms are included in their definition. The renormalization conditions will be formulated in the next subsection as inductive assumptions. The effect of subsequent renormalization steps is that the variable $\psi_{j}$ is replaced by the corresponding functions $\psi_{k}^{(j)}$. Each renormalization step increases the index $k$ by 1 . The functions $\mathscr{E}^{(j)}$ and $\psi_{k}^{(j)}$ are also rescaled to the $L^{j} \eta$-scale, but the only effect of this scaling operation is that the lattice $T_{1}^{(j)}$ is rescaled to $T_{L^{j} \eta}^{(j)}$, and the functions are defined on the rescaled lattice in the same way as they were defined on the original unit lattice. Thus in the second equality above it does not matter what is a scale of the lattice $T^{(j)}$.

Consider the functions $\mathscr{E}^{(j)}\left(y ; \psi_{j}, v_{j} h\right)$. They have three important properties: analyticity in $\psi_{j}, h$, symmetries and localizability. We shall describe these properties in detail as further inductive assumptions.

To formulate the analyticity properties we have to introduce at first some spaces of spin configurations. It is important to consider spin configurations together with external field vectors $h$. We start with a space of configurations $\phi$ on the lattice $T_{\xi}$, $\xi=L^{-j}$, with values in $\mathbf{R}^{N}$, and of vectors $h$ from the space $\mathbf{R}^{N}$. We define

$$
\begin{aligned}
\Phi_{j}(\sigma, \varepsilon ; \lambda, v) \\
\quad=\left\{(\phi, h):\left|\partial^{\xi} \phi\right|<\sigma \varepsilon,\left|\Delta^{\xi} \phi\right|<\sigma^{2} \varepsilon,|\alpha|<\sigma^{2} \varepsilon,|\phi-h|<\frac{\sigma \varepsilon}{\sqrt{v}},\left.|| h\right|^{2}-1 \mid<\frac{\sigma^{2} \varepsilon^{2}}{v}\right\}, \\
\alpha=\frac{\lambda}{2}\left(|\phi|^{2}-1\right),
\end{aligned}
$$


where $\sigma, \varepsilon, \lambda, v$ are positive constants. This definition is motivated by properties of the minimal configurations $\phi_{j}$. The space is defined in a natural way on the lattice $T_{\xi}$, but we may rescale the lattice and the configurations to any other scale, and we obtain the space defined on the new lattice. It is still defined by the same conditions as in (2.15), formulated in terms of the scale $\xi$. If we want to write these conditions with derivatives for the new scale, we have to introduce corresponding factors on the right-hand sides. We extend the above real space to a complex space of configurations $\left(\phi+\phi^{\prime}, h+h^{\prime}\right)$, where $\phi$ and $h$ are as above, $\phi^{\prime}$ is defined on $T_{\xi}$ and has values in $\mathbf{C}^{N}, h^{\prime}$ is defined on $T_{1}^{(j)}$ and has values in $\mathbf{C}^{N}$. We consider $h+h^{\prime}$ as a function on $T_{1}^{(j)}$, and we define

$$
\begin{gathered}
\Phi_{j}^{c}(\sigma, \varepsilon ; \lambda, v)=\left\{\left(\phi+\phi^{\prime}, h+h^{\prime}\right):(\phi, h) \in \Phi_{j}(\sigma, \varepsilon ; \lambda, v)\right. \\
\left.\left|\phi^{\prime}\right|<\varepsilon,\left|\partial^{\xi} \phi^{\prime}\right|<\sigma \varepsilon,\left|\Delta^{\xi} \phi^{\prime}\right|<\sigma^{2} \varepsilon,|\delta \alpha|<\sigma^{2} \varepsilon,\left|h^{\prime}\right|<\frac{\sigma^{2} \varepsilon}{v}, \quad\left|\phi \cdot h^{\prime}\right|<\frac{\sigma^{2} \varepsilon^{2}}{v}\right\} \\
\delta \alpha=\frac{\lambda}{2}\left(\left(\phi+\phi^{\prime}\right)^{2}-\phi^{2}\right)=\frac{\lambda}{2}\left(2 \phi \cdot \phi^{\prime}+\phi^{\prime 2}\right) .
\end{gathered}
$$

In the future it will be convenient to use conditions on configurations $\phi+\phi^{\prime}$ directly. The conditions in (2.15), (2.16) obviously imply the following ones:

$$
\begin{gathered}
\left|\phi^{\prime}\right|<\varepsilon,\left|\partial^{\xi}\left(\phi+\phi^{\prime}\right)\right|<2 \sigma \varepsilon \\
\left|\Delta^{\xi}\left(\phi+\phi^{\prime}\right)\right|<2 \sigma^{2} \varepsilon,|\alpha+\delta \alpha|<2 \sigma^{2} \varepsilon,\left|h^{\prime}\right|<\frac{\sigma^{2} \varepsilon}{v},\left|\phi \cdot h^{\prime}\right|<\frac{\sigma^{2} \varepsilon^{2}}{v}, \\
\alpha+\delta \alpha=\frac{\lambda}{2}\left(\left(\phi+\phi^{\prime}\right)^{2}-1\right) .
\end{gathered}
$$

In fact the above conditions are equivalent to (2.16), except some unimportant numerical factors on the right sides. We will use the spaces $\Phi_{j}^{c}(\sigma, \varepsilon ; \lambda, \nu)$ in the case when $\lambda=\lambda_{j}, v=v_{j}$. In this case the condition on the first derivative in (2.16) or (2.17) can be strengthened, but for the longitudinal derivative only. For simplicity let us write $\phi$ instead of $\phi+\phi^{\prime}$, thus $\phi$ has values in $\mathbf{C}^{N}$. We have the equality.

$$
\begin{aligned}
\phi(x) \cdot\left(\partial^{\xi} \phi\right)\left(\left\langle x, x^{\prime}\right\rangle\right) & =\frac{1}{\xi} \phi(x) \cdot\left(\phi\left(x^{\prime}\right)-\phi(x)\right) \\
& =-\frac{1}{2 \xi}\left(\phi\left(x^{\prime}\right)-\phi(x)\right)^{2}+\frac{1}{2 \xi}\left(\phi^{2}\left(x^{\prime}\right)-\phi^{2}(x)\right) \\
& =-\frac{1}{2} \xi\left(\partial^{\xi} \phi\right)^{2}\left(\left\langle x, x^{\prime}\right\rangle\right)+\frac{1}{\lambda \xi}\left[\frac{\lambda}{2}\left(\phi^{2}\left(x^{\prime}\right)-1\right)-\frac{\lambda}{2}\left(\phi^{2}(x)-1\right)\right],
\end{aligned}
$$

which implies the bound

$$
\left|\phi \cdot \partial^{\xi} \phi\right|<2 \xi \sigma^{2} \varepsilon^{2}+\frac{4}{\lambda \xi^{2}} \xi \sigma^{2} \varepsilon<5 \xi \sigma^{2} \varepsilon
$$


assuming that $\varepsilon$ is sufficiently small, e.g. $\varepsilon<\frac{1}{10}$, and for $\lambda=\lambda_{j}$ the constant $\lambda_{j} \xi^{2}$, which is close to $\lambda_{0}$ by (2.5), should be greater than a constant close to 1 , e.g. $\lambda, \breve{\zeta}^{2}>\frac{5}{6}$. The spaces $\Phi_{j}^{c}$ are natural analyticity domains for functions of minimal configurations $\phi_{j}$. In particular we will construct the solution $\phi_{k}$ of the variational problem (2.2) in the space $\Phi_{k}\left(1, c_{0} ; \lambda, v\right)$ for $c_{0}$ sufficiently small.

To consider functions of $\left(\psi_{j}, h\right)$ we have to introduce new spaces. The configurations $\psi_{j}$ are defined on the lattice $T^{(j)}$ and have values in $\mathbf{R}^{N}$, the vectors $h$ belong to the unit sphere $S^{N-1}$. We define

$$
\begin{aligned}
\Xi_{J}(\sigma, \varepsilon)= & \left\{\left(\psi_{j}, h\right):\left(\phi_{J}\left(\psi_{j} ; h, a_{j}, \lambda_{J}, v_{j}\right), h\right) \in \Phi_{j}\left(\sigma, \varepsilon ; \lambda_{j}, v_{j}\right),\right. \\
& \left|\psi_{J}-Q_{J} \phi_{j}\left(\psi_{j} ; h, a_{j}, \lambda_{j}, v_{j}\right)\right|<\sigma^{2} \varepsilon_{j} .
\end{aligned}
$$

We have assumed implicitly in the above definition that $\left(\psi_{j}, h\right)$ belongs to a domain of the function $\phi_{j}\left(\psi_{j}, h\right)$. We will see that the conditions above imply stronger restrictions on $\left(\psi_{j}, h\right)$, assuming that $\varepsilon$ and $\sigma \varepsilon$ are sufficiently small. We assume that after $k$ steps the constants $a_{j}, \lambda_{j}, v_{j}$ are given by the equalities (2.9), (2.11), so the space $\Xi_{j}(\sigma, \varepsilon)$ depends actually on $k$ also. We do not write this dependence explicitly for the same reason as before. We extend the above real space to a complex space of configurations $\left(\psi_{j}+\psi_{j}^{\prime}, h+h^{\prime}\right), \psi_{j}^{\prime}, h^{\prime}$ are defined on $T^{(j)}$ and have values in $\mathbf{C}^{N}$. This space is defined by

$$
\begin{gathered}
\Xi_{j}^{c}(\sigma, \varepsilon)=\left\{\left(\psi_{j}+\psi_{j}^{\prime}, h+h^{\prime}\right):\left(\psi_{j}, h\right) \in \Xi_{j}(\sigma, \varepsilon),\right. \\
\left(\phi_{j}\left(\psi_{j}+\psi_{j}^{\prime} ; h+h^{\prime}\right), h+h^{\prime}\right)=\left(\phi_{j}\left(\psi_{j}, h\right)+\delta \phi_{j}\left(\psi_{j}^{\prime}, h^{\prime}\right), h+h^{\prime}\right) \in \Phi_{j}^{c}\left(\sigma, c ; \lambda_{j}, v_{j}\right), \\
\left.\left|\psi_{j}^{\prime}-Q_{j} \delta \phi_{j}\left(\psi_{j}^{\prime}, h^{\prime}\right)\right|<\sigma^{2} \varepsilon\right\}
\end{gathered}
$$

where we have dropped the dependence on $a_{j}, \lambda_{j}, v_{j}$ in the functions $\phi_{j}, \delta \phi_{j}$. The last function is defined by the equality in the definition. We have used the fact that the function $\phi_{j}\left(\psi_{j} ; h\right)$ has an analytic extension $\phi_{j}\left(\psi_{j}+\psi_{j}^{\prime}, h+h^{\prime}\right)$ to some absolutely defined domain, which will be described in the next paper when we will prove this statement. We have assumed again implicitly in the above definition that the configurations $\left(\psi_{j}+\psi_{j}^{\prime}, h+h^{\prime}\right)$ belong to this domain. Notice that in the definitions (2.15), (2.16), (2.20), (2.21) not all conditions are independent, for example the conditions involving the Laplace operator $\Delta^{\xi}$ are consequences of the others. We have listed all conditions which are explicitly used in the renormalization analysis.

Now we can formulate the next inductive hypothesis:

(H.3) the functions $\mathscr{E}^{(j)}\left(y ; \psi_{J}, v, h\right)$ are defined on the space $\Xi_{J}\left(1, \varepsilon_{j}\right)$, and have analytic extensions defined on the space $\Xi_{j}^{c}\left(1, \varepsilon_{j}\right)$, where $\varepsilon_{j}=\alpha_{0, k} \xi^{\frac{d-2}{2}-y}$ $\left(A_{0}+\log \xi^{-1}\right)^{p}, \alpha_{0 . k}=\alpha_{0}\left(1+\sum_{n=k}^{\infty} \frac{1}{n^{2}}\right), 0 \leqq \gamma<\gamma_{0} \leqq \frac{d-2}{2}$.

Let us make a few comments on the constants above. The spaces increase with increasing $\gamma$, and the two extremal cases are especially interesting. For $\gamma=0$ we have the smallest space, and we take $p=p_{0}$ and $A_{0}$ as in the definition (1.15). The space contains then the corresponding small field domain. In this case we obtain best bounds for the actions $\mathscr{E}^{(j)}$, which yield a uniform bound for $\mathscr{E}_{k}$. With $\gamma$ increasing the spaces and the bounds increase also, and we define $\gamma_{0}$ as the upper bound of these $\gamma$ 's for which we still have a uniform bound for $\mathscr{E}_{k}$. We will find $\gamma_{0}$ explicitly. For $\gamma$ close to $\gamma_{0}$ we have largest spaces, and therefore best analyticity properties. We choose $\gamma$ depending on a problem we want to solve. To study decay properties of correlation functions we will need the largest analyticity domains, so 
we consider $\gamma$ close to $\gamma_{0}$. In this case we do not need the logarithmic factors and we take $p=0$. In the future we will consider for simplicity this case only, although all constructions and bounds are valid for all $\gamma$ in the interval $\left[0, \gamma_{0}\left[\right.\right.$. The constant $\alpha_{0}$ must be chosen sufficiently small in order to satisfy various restrictions connected with our analysis.

We have not yet explained a reason for introducing the parameter $\sigma$ in the definitions (2.15), (2.16), (2.20), (2.21). It is a very important parameter; it measures scaling properties of bounds for corresponding expressions, in particular improvements of the bounds with increasing $k$. This fundamental property is clarified in the following lemma.

Lemma 2.1. The function $\psi_{k}^{(j)}$ determines an analytic mapping of the space $\Xi_{k}^{c}(\sigma, \varepsilon)$ into the space $\Xi_{j}^{c}\left(L^{j} \eta \sigma, \varepsilon\right)$, i.e.

$$
\psi_{k}^{(j)}:\left(\psi+\psi^{\prime}, h+h^{\prime}\right) \in \Xi_{k}^{c}(\sigma, \varepsilon) \rightarrow\left(\psi_{k}^{(j)}\left(\psi+\psi^{\prime}, h+h^{\prime}\right), h+h^{\prime}\right) \in \Xi_{j}^{c}\left(L^{j} \eta \sigma, \varepsilon\right)
$$

for $\varepsilon$ sufficiently small, $\sigma \leqq 1$.

A proof of this lemma follows immediately from the composition property (2.8), the equalities (2.14), (2.9), (2.10), (2.11), and the definitions of the spaces $\Xi_{j}^{c}$. Let us remark that the lemma gives a main reason for introducing these spaces; they are matched with properties of the functions $\psi_{k}^{(j)}$ in the arguments of the functions $\mathscr{E}^{(j)}$. We apply the lemma in the case $\sigma=1, \varepsilon=\varepsilon_{k}$. The values of $\psi_{k}^{(j)}$ are then in the space $\Xi_{j}^{c}\left(L^{j} \eta, \varepsilon_{k}\right)=\Xi_{j}^{c}\left(L^{j} \eta, \frac{\varepsilon_{k}}{\varepsilon_{j}} \varepsilon_{j}\right)$, and in comparison with $\Xi_{j}^{c}\left(1, \varepsilon_{j}\right)$ we improve the bounds by corresponding powers and products of the factors $L^{j} \eta$ and $\frac{\varepsilon_{k}}{\varepsilon_{j}}$. This is a basis of the whole renormalization analysis.

Let us formulate now assumptions on symmetry properties of the functions $\mathscr{E}^{(j)}\left(y ; \psi_{j}, v_{j} h\right)$. Let $r$ denote a Euclidean transformation of the lattice $T^{(j)}$ into itself. Such transformations are generated by translations, reflections and permutations of coordinates. The transformation $r$ determines a transformation of configurations on $T^{(j)}$ in the usual way, e.g. $\left(r \psi_{j}\right)(y)=\psi_{j}\left(r^{-1} y\right), y \in T^{(j)}$. We assume that

$$
\begin{aligned}
\mathscr{E}^{(j)}\left(r y ; r \psi_{j}, v_{j} h\right) & =\mathscr{E}^{(j)}\left(y ; \psi_{j}, v_{j} h\right), \\
\mathscr{E}^{(j)}\left(y ; R \psi_{j}, v_{j} R h\right) & =\mathscr{E}^{(j)}\left(y ; \psi_{j}, v_{j} h\right), \quad R \in O(N),
\end{aligned}
$$

hold on $\Xi_{j}\left(1, \varepsilon_{j}\right)$ and the same equalities hold for the analytic extensions of the functions $\mathscr{E}^{(j)}$, the second on the space $\Xi_{j}^{c}\left(1, \varepsilon_{j}\right)$, the first on the subspace of this space with constant configurations $h+h^{\prime}$.

Of course the Euclidean transformations $r$ and the orthogonal transformations $R$ leave invariant the spaces and the subspace. The above symmetries play a crucial role in the renormalization analysis, in particular the second one. The first has two important implications. For Euclidean transformations $r$ leaving a point $y \in T^{(j)}$ invariant, i.e. $r y=y$, we have

$$
\mathscr{E}^{(j)}\left(y ; r \psi_{j}, v_{j} h\right)=\mathscr{E}^{(j)}\left(y ; \psi_{j}, v_{j} h\right) .
$$

This symmetry is used to determine a final form of renormalization conditions in the next subsection. Taking constant configurations $\left(\psi_{j}, h\right)$, e.g. $\psi_{j}(y)=\psi$, and taking $r$ as a translation by a vector $z \in T^{(j)}$ we obtain

$$
\mathscr{E}^{(j)}\left(y+z ; \psi, v_{j} h\right)=\mathscr{E}^{(j)}\left(y ; \psi, v_{j} h\right) .
$$


Thus all the functions $\mathscr{E}^{(j)}\left(y ; \psi, v_{j} h\right)$ are equal for $y \in T^{(j)}$, and we define an effective potential for the action $\mathscr{E}^{(j)}$ by the equality

$$
\mathscr{\psi}^{\cdot(j)}\left(\psi, v_{j} h\right)=\mathscr{E}^{(J)}\left(y ; \psi, v_{j} h\right) \text {. }
$$

The effective potential has an analytic extension on a domain in $\mathbf{C}^{N} \times \mathbf{C}^{N}$ which may be identified with a set of constant configurations in $\Xi_{j}^{c}\left(1, \varepsilon_{j}\right)$, and it is invariant with respect to orthogonal transformations, i.e. it satisfies the second equality in (H.4).

The third property of the effective actions $\mathscr{E}^{(j)}\left(y ; \psi_{j}, v_{j} h\right)$ is their localizability. It has basically a standard form, e.g. see Sect. 0 in [2d]. At first we define a class $\mathscr{Z}_{j}$ of localization domains of the lattice $T_{\xi}$. We take a partition of $T_{\xi}$ into large cubes, i.e. cubes of size $M=L^{m}$, where $m$ is a sufficiently large integer. We require that partitions taken for various scales are compatible with each other. It is convenient to identify domains in lattices with properly defined domains in the continuous space $\mathbf{R}^{d}$, or rather in the corresponding torus. A simple way to do it is to consider the torus $T_{1}$ as a subset of the lattice $\mathbf{Z}^{d}+\left(\frac{1}{2}, \frac{1}{2}, \ldots, \frac{1}{2}\right)$, and to assign to a point $x \in T_{1}$ the unit cube of $\mathbf{R}^{d}$ with center at the point $x$ and with vertices at points of the unit lattice $Z^{d}$. We identify a subset $X$ of the lattice with a union of cubes corresponding to points of the subset. A partition of $T_{1}$ into large cubes can be obtained by taking a partition of $\mathbf{R}^{d}$ into cubes determined by points of the lattice $M \mathbf{Z}^{d}$, and all subsequent partitions are determined by the lattices $L^{j} M \mathbf{Z}^{d}$ and rescaled to the corresponding $\xi$-scales. We denote them correspondingly by $\pi_{j}$. For such a partition we construct another partition, or rather a cover $\pi_{j}^{\prime}$ by LM-cubes which are unions of $L^{d}$ of the corresponding $M$-cubes. For the cover $\pi_{j}^{\prime}$ of the lattice $T_{\xi}$ we define a class $\mathscr{Z}_{j}$ as a class of domains $X$ which are connected unions of open cubes from $\pi_{j}^{\prime}$, i.e. such that

$$
X=\bigcup_{\square \in \pi_{i}^{\prime}: \square \subset x} \square .
$$

The cubes in the above decomposition may overlap. We consider $X$ as an open domain in $\mathbf{R}^{d}$, and we assume that it is connected. The class $\mathscr{Z}_{j}$ is defined for the lattice $T_{\xi}$ in the scale $\xi$, and if this lattice is rescaled, then domains in $\mathscr{Z}$, are also rescaled in the same way. For domains $X \in \mathscr{D}$, in the scale $\xi$ we define a function $d_{j}(X)$ which measures a tree size of $X$. We consider $X$ as a domain in the continuous space, and we define

$$
\begin{aligned}
d_{j}(X)= & \inf \left\{M^{-1}|\Gamma|: \Gamma\right. \text { is a continuous tree graph } \\
& \text { contained in } \left.X \text { and intersecting every cube } \square \subset X, \square \in \pi_{j}^{\prime}\right\} .
\end{aligned}
$$

This definition refers explicitly to the scale $\xi$. If a domain $X \in \mathscr{Z}_{j}$ is considered in a different scale we can define $d_{j}(X)$ either by rescaling $X$ back to the $\xi$-scale and using the above definition, or writing the above definition with an additional scaling factor. We can formulate now the next inductive assumption

$$
\mathscr{E}^{(j)}\left(y ; \psi_{j}, v_{j} h\right)=\sum_{X \in \mathcal{S}, i v \in X} \mathscr{E}^{(j)}\left(y, X ; \psi_{J}, v_{j} h\right),
$$

where the functions $\mathscr{E}^{(j)}\left(y, X ; \psi_{j}, v_{j} h\right)$ have analytic extensions defined on $\Xi_{j}^{c}\left(1, c_{j}\right)$; these analytic extensions depend on $\left(\psi_{J}+\psi_{j}^{\prime}, h+h^{\prime}\right)$ restricted to the corresponding domains $X$ and satisfy the bounds

$$
\left|\mathscr{E}^{(j)}\left(y, X ; \psi_{j}+\psi_{j}^{\prime}, v_{j}\left(h+h^{\prime}\right)\right)\right| \leqq E_{0} \exp \left(-\kappa d_{j}(X)\right),
$$


where $E_{0}, \kappa$ are positive constants, $\kappa$ can be chosen arbitrarily large, depending on the large cube size $M$. The analytic extensions are invariant with respect to orthogonal transformations $R \in O(N)$, i.e. they satisfy the second equality in (H.4).

The above expansion, called a localization expansion, plays a fundamental role in the renormalization analysis, and one of the main steps in the presented method is an inductive construction of such an expansion for a new contribution to the effective action determined by the $k+1^{\text {st }}$ renormalization transformation. This construction will be described in the third paper. It will also yield some additional properties, e.g. an Euclidean covariance of the localized actions as in the first equality in (H.4), only on the left-hand side the transformation $r$ has to be applied to $X$ also. This property is not important here. The constant $\kappa$ depends on the size $M$, and this dependence will be described precisely later on. We fix $\kappa$ and $M$ by several conditions they have to satisfy, which will be formulated successively in the future. The most important condition is a convergence of the series below

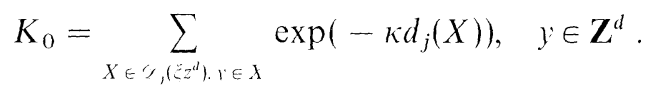

The class of localization domains above is defined for the whole lattice $\xi \mathbf{Z}^{d}$, and it is well known that the series is convergent for $\kappa$ sufficiently large, e.g. $k \geqq \kappa_{0}$, and the sum is bounded by a constant depending on $d$ only. The sum above does not depend on the point $y$, so $K_{0}$ is a function of $\kappa$ only. In the future we will use $K_{0}$ defined for $\kappa_{0}$, so we will have a bound from above by $K_{0}$ instead of equality (2.28). As a simple application of this bound we obtain

$$
\left|\mathscr{C}^{(j)}\left(y, \psi_{J}, v_{j} h\right)\right| \leqq E_{0} K_{0}
$$

on the space $\Xi_{j}^{c}\left(1, \varepsilon_{j}\right)$. Here $\psi_{j}, h$ denote, for simplicity, elements of the complex space. In the future we will use frequently this simplified notation. The above bound implies that the $\mathrm{j}^{\text {th }}$ contribution $\mathscr{E}^{(j)}$ to the effective action can be bounded by $E_{0} K_{0}\left|T_{1}^{(j)}\right|=E_{0} K_{0}\left|T_{1}\right| L^{-j d}$, so for the whole action $\mathscr{E}_{k}$ we get

$$
\left|\mathscr{E}_{h}\right| \leqq \sum_{j=1}^{k} E_{0} K_{0}\left|T_{1}\right| L^{-j d}<\left(L^{d}-1\right)^{-1} E_{0} K_{0}\left|T_{1}\right| .
$$

From the renormalization group point of view it is a very bad bound; after $k$ steps we would like to have a bound by a constant times $\left|T_{1}^{(k)}\right|=\left|T_{n}\right|=\eta^{d}\left|T_{1}\right|$. Such an improvement is connected with a renormalization of the effective action we have to do after each step. This renormalization is discussed in the third paper. Here we assume that the effective action is properly renormalized, and this can be expressed in the form of some renormalization conditions. We will formulate these conditions now.

The first set of conditions concerns the effective potential $\psi^{\cdot(j)}\left(\psi, v_{j} h\right)$ defined by (2.25). We assume that

$$
\begin{aligned}
& y^{\cdot(J)}\left(e_{1}, v_{j} e_{1}\right)=0,\left(\frac{\partial}{\partial h} y^{(j)}\right)\left(e_{1}, v_{j} e_{1}\right) \cdot e_{1}=0, \quad e_{1}=(1,0, \ldots, 0), \\
& \left(\frac{\partial}{\partial \psi} y^{\cdot(j)}\right)\left(e_{1}, v_{j} e_{1}\right) \cdot e_{1}=\sum_{v^{\prime} \in T^{\prime \prime \prime}}\left(\frac{\partial}{\partial \psi\left(y^{\prime}\right)} \mathscr{E}^{(j)}\right)\left(y ; e_{1}, v_{j}^{\prime} e_{1}\right) \cdot e_{1}=0
\end{aligned}
$$

for all $j \leqq k$. 
The second condition involves the second order differential of the function $\mathscr{E}^{(j)}\left(\psi, v_{j} h\right)$ with respect to the spin variable $\psi$. We assume that

$$
\sum_{x \in Z^{d}} \operatorname{tr}\left[\lim _{T_{\xi} \rightarrow \xi \mathbf{Z}^{d}}\left(\frac{\partial^{2}}{\partial \psi(x) \partial \psi(0)} \mathscr{E}^{(j)}\right)\left(e_{1}, v_{j} e_{1}\right) \cdot\left(e_{2} \otimes e_{2}\right)\right]|x|^{2}=0 .
$$

A role and the form of the above conditions will become clear in the next section. The constants on the left-hand sides will appear as coefficients at relevant and marginal expressions, as usual in the renormalization group approach. A meaning of these concepts, the relevant, marginal, irrelevant expressions, will be also explained in the next section.

We have to introduce one more assumption, a more precise description of the asymptotic behavior (2.5). It is not uniquely defined; it depends on how we formulate renormalization group equations for the coefficients $\beta_{k}, a_{k}, \lambda_{k}, v_{k}$. It turns out that we do not need all these coefficients for the renormalization procedure, so we have freedom of choice of some additional equations. Depending on this choice we have different precise forms of the asymptotic behavior, but they all have the general form (2.5). We will discuss all these issues in detail in the third paper. Here we formulate the following particularly simple assumption on those coefficients:

$$
\beta_{k}=\beta L^{k(d-2)} \gamma_{k}, \quad a_{k}=a \frac{1-L^{-2}}{1-L^{-2 k}}, \quad \lambda_{k}=\lambda L^{2 k}, \quad v_{k}=v L^{2 k} \delta_{k},
$$

and the constants $\gamma_{k}, \delta_{k}$ are arbitrarily close to 1 if $\beta$ is sufficiently large.

From this assumption it follows that $a_{k}$ and $\lambda_{k}$ are determined by the free renormalization group flow (2.10), (2.11) and the pure scaling (2.9) correspondingly, but our whole analysis in this and the subsequent papers does not depend on this assumption. In the third paper we will formulate more precise inductive assumptions on the constants $\gamma_{k}, \delta_{k}$, from which it will follow in particular that there exist limits $\lim _{k \rightarrow \infty} \gamma_{k}=\gamma_{\infty}, \lim _{k \rightarrow \infty} \delta_{k}=\delta_{\infty}$. This was our last hypothesis, and we have completed the inductive description of the effective actions.

We describe now the second term in the exponential in (H.1). This term determines a generating functional for correlation functions, and here we describe minimal assumptions which can be reproduced by the renormalization transformations, and which are sufficient to control a convergence as $T \rightarrow \mathbf{Z}^{d}$ and $v \rightarrow 0+$, or $k \rightarrow \infty$. They will allow us to establish existence of the correlation functions in these limits, hence to establish existence of the corresponding thermodynamic phases, but they are not enough to prove more detailed properties of the correlation functions. Such properties will be proved in another paper, in collaboration with M. O'Carroll, based on a much more elaborate description of this term. Now we assume that

$$
\begin{gathered}
\mathscr{F}_{k}\left(\psi_{k}, h, g\right)=\left\langle g, \phi_{k}\right\rangle_{1}+\sum_{j=1}^{k} \beta_{j-1}^{-\frac{1}{2}} \mathscr{F}^{(j)}\left(\psi_{k}^{(j)}\left(\psi_{k}, v_{k} h\right), v_{j} h, g\right), \\
\mathscr{F}^{(j)}\left(\psi_{j}, v_{j} h, g\right)=\left\langle g, \mathscr{M}^{(j)}\left(\psi_{j}, v_{j} h, g\right)\right\rangle_{1},
\end{gathered}
$$

$\mathscr{M}^{(j)}\left(x ; \psi_{j}, v_{j} h, g\right), x \in T$, have analytic extensions defined on the space $\Xi_{j}^{c}\left(1, \varepsilon_{j}\right) \times\left\{g: g\right.$ are defined on $T$ and have values in $\left.\mathbf{C}^{N},\|g\|_{l^{1}}<1\right\}$, and these 
analytic functions have corresponding invariance and localizability properties as in (H.4),(H.5). Terms of the localization expansions satisfy the bounds

$$
\left|\mathscr{M}^{(j)}\left(x, X ; \psi_{j}, v_{j} h, g\right)\right| \leqq M_{0} \xi^{\gamma-2 x_{1}} \exp \left(-\kappa d_{j}(X)\right),
$$

where $M_{0}$ is a positive constant and $\alpha_{1}$ is a sufficiently small positive constant.

The above bounds imply in particular that the sum over $j$ in (H.9) can be bounded by $M_{0} K_{0} \beta^{-\frac{1}{2}}$, so it is small for $\beta$ sufficiently large. Notice also that invariance with respect to the orthogonal transformations $R \in O(N)$ means actually covariance for the functions $\mathscr{M}^{(j)}$, i.e. if $\psi_{j}, h, g$ are transformed simultaneously by $R$, then $\mathscr{M}^{(j)}$ is also transformed by $R$. This follows from the invariance of the corresponding function $\mathscr{F}^{(j)}$.

The last element of the effective density $\rho_{k}$ in (H.1) to define is the characteristic function $\chi_{k}$. It is given by

$$
\begin{gathered}
\chi_{k}=\chi\left(\left\{\left|\psi_{k}-Q_{k} \phi_{k}\right|<\beta_{k}^{-\frac{1}{2}} p\left(\beta_{k}\right) \text { on } T_{1}^{(k)},\left|\partial^{\eta} \phi_{k}\right|<\beta_{k}^{-\frac{1}{2}} p\left(\beta_{k}\right)\right.\right. \\
\left.\left.\left|\Delta^{\eta} \phi_{k}\right|<\beta_{k}^{-\frac{1}{2}} p\left(\beta_{k}\right),\left|\alpha_{k}\right|<\beta_{k}^{-\frac{1}{2}} p\left(\beta_{k}\right),\left|\phi_{k}-h\right|<v_{k}^{-\frac{1}{2}} \beta_{k}^{-\frac{1}{2}} p\left(\beta_{k}\right) \text { on } T_{\eta}\right\}\right) \\
\alpha_{k}=\frac{\lambda_{k}}{2}\left(\left|\phi_{k}\right|^{2}-1\right)
\end{gathered}
$$

The domain defined by the above characteristic function can be identified with the subspace of $\Xi_{k}\left(1, \beta_{k}^{-\frac{1}{2}} p\left(\beta_{k}\right)\right)$ corresponding to a fixed unit sphere vector $h$.

We have finished the description of the effective densities $\rho_{k}$, but there are still some undefined elements in their definition (1.16), namely the characteristic functions $\chi^{(j)}$. Their exact definitions are quite technical and we postpone them to the third paper, but let us make a few explanatory comments now. The functions $\chi^{(j)}$ give restrictions on fluctuation variables at corresponding steps. Once the variables are defined, the restrictions are very simple, just on the magnitude of the variables. The definition is inductive and technically involved, and although it could be formulated here it is much more natural to do it at a proper place in the third paper.

We can formulate now the main result of this part of the work connected with the small field analysis.

Theorem 2.2. Consider the model given by (1.1), (1.5) for $d \geqq 3, N \geqq 2, \beta>0$, $1 \leqq \lambda \leqq+\infty, 0<v \leqq 1$, and define the sequence of densities $\rho_{k}$ applying the small field renormalization transformations (1.16). For $\beta$ sufficiently large there exist constants $\beta_{k}, a_{k}, \lambda_{k}, v_{k}, E_{0}, M, \kappa, \alpha_{0}, \gamma, M_{0}, \alpha_{1}$ such that the densities $\rho_{k}$ satisfy the inductive hypotheses (H.1)-(H.9), as long as $v_{k} \leqq 1$.

This theorem will be proved in several stages in the three papers. The proof will provide many detailed estimates of terms of the effective actions, and conditions on various constants. In particular we will construct renormalization group equations for the coefficients $\beta_{k}, a_{k}, \lambda_{k}, v_{k}$ which will determine them uniquely in terms of the initial coefficients. In this paper we analyze consequences of the inductive hypotheses (H.1)-(H.8) and we obtain improved estimates of the effective actions $\mathscr{E}^{(j)}$ and 
$\mathscr{E}_{k}$. This analysis is called a renormalization analysis because of a crucial role played by the renormalization conditions (H.6),(H.7). It is a subject of the next section.

\section{Renormalization Analysis and Bounds on the Effective Actions}

In this section we describe in detail the renormalization analysis, which could be called also a renormalization expansion, and with its help we prove uniform bounds of the effective actions $\mathscr{E}_{k}$ by volumes $\left|T_{\eta}\right|=\left|T_{1}^{(k)}\right|$. The main results are formulated in Proposition 3.4, Corollary 3.5, Proposition 3.10, and Corollary 3.11.

Before we start this analysis we have to introduce some new spaces of configurations $\left(\psi_{j}, h\right)$.

The spaces $\Xi_{j}^{c}(\sigma, \varepsilon)$ are crucial for inductive constructions of this paper, but the fact that we have to use the non-linear functions $\phi_{j}$ in their definition makes them difficult to work with, it is difficult to perform various operations on configurations $\psi_{j}$. For such purposes we have to find another, more convenient characterization of these spaces, formulated directly in terms of the configurations $\left(\psi_{j}, h\right)$. Let us study at first some implications of the definitions (2.15), (2.16), (2.20), (2.21) for those configurations. We have

$$
\begin{aligned}
\left|\left(\partial^{1} \psi_{j}\right)(b)\right| \leqq & \left|\psi_{j}\left(b_{+}\right)-\left(Q_{j} \phi_{j}\right)\left(b_{+}\right)\right|+\left|\left(Q_{j} \phi_{j}\right)\left(b_{+}\right)-\left(Q_{j} \phi_{j}\right)\left(b_{-}\right)\right| \\
& +\left|\psi_{j}\left(b_{-}\right)-\left(Q_{j} \phi_{j}\right)\left(b_{-}\right)\right|<\sigma^{2} \varepsilon+\sigma \varepsilon+\sigma^{2} \varepsilon \leqq 3 \sigma \varepsilon,
\end{aligned}
$$

where we have used the restriction $\sigma \leqq 1$, and where $b=\left\langle b_{-}, b_{+}\right\rangle$is a bond of the lattice $T^{(j)}$. For a locally longitudinal derivative, i.e. a derivative in the direction of $\psi_{j}$, we have

$$
\begin{aligned}
& \psi_{j}(y) \cdot\left(\partial^{1} \psi_{j}\right)\left(\left\langle y, y^{\prime}\right\rangle\right)=\left(\psi_{j}(y)-\left(Q_{J} \phi_{j}\right)(y)\right) \cdot\left(\hat{c}^{1} \psi_{j}\right)\left(\left\langle y, y^{\prime}\right\rangle\right) \\
& +\left(Q_{,} \phi_{j}\right)(y) \cdot\left(\partial^{1}\left(\psi_{j}-Q_{j} \phi_{j}\right)\right)\left(\left\langle y, y^{\prime}\right\rangle\right) \\
& -\sum_{x, V^{\prime} \in B_{,}(1)} \xi^{2 d} \sum_{b=\left\langle x^{\prime}, \Upsilon^{\prime}+\left(1^{\prime}-1\right)\right\rangle} \xi\left(\partial^{z} \phi_{j}\right)\left(\Gamma_{x, x^{\prime}} \cup\left\langle x^{\prime}, b_{-}\right\rangle\right) \\
& \text {- }\left(\hat{c}^{\xi} \phi_{J}\right)(b) \\
& +\sum_{r^{\prime} \in B_{,}(1)} \xi^{d} \sum_{b c\left\langle r^{\prime} x^{\prime}+\left(y^{\prime}-1\right)\right\rangle} \xi \phi_{J}\left(b_{-}\right) \cdot\left(\hat{c}^{\xi} \phi_{j}\right)(b),
\end{aligned}
$$

where $\Gamma_{x, x^{\prime}}$ is a shortest path of the lattice connecting the points $x, x^{\prime}$. Hence

$$
\begin{aligned}
\left|\psi,(y) \cdot\left(\hat{c}^{1} \psi_{j}\right)\left(\left\langle y, y^{\prime}\right\rangle\right)\right|< & 3 \sigma^{3} \varepsilon^{2}+\left(1+2 \frac{\sigma^{2} \varepsilon}{\lambda_{j}}\right) 2 \sigma^{2} \varepsilon+(d+1) \sigma^{2} \varepsilon^{2} \\
& +5 \xi \sigma^{2} \varepsilon<5 \sigma^{2} \varepsilon
\end{aligned}
$$

for $\varepsilon$ sufficiently small, e.g. $(d+5) \varepsilon \leqq 1$. Next

$$
\begin{aligned}
\psi_{j}^{2}(y)-1= & \left(\psi_{j}(y)-\left(Q_{j} \phi_{j}\right)(y)\right)^{2}+2\left(\psi_{j}(y)-\left(Q_{j} \phi_{j}\right)(y)\right) \cdot\left(Q_{j} \phi_{j}\right)(y) \\
& -\frac{1}{2} \sum_{x^{\prime} \in B_{,}(t)} \xi^{2 d}\left(\left(\partial^{\check{\xi}} \phi_{j}\right)\left(\Gamma_{x \cdot x^{\prime}}\right)\right)^{2}+\left(Q_{J}\left(\phi_{j}^{2}-1\right)\right)(y),
\end{aligned}
$$


hence

$$
\begin{aligned}
\left|\psi_{j}^{2}(y)-1\right| & <\sigma^{4} \varepsilon^{2}+2 \sigma^{2} \varepsilon\left(1+2 \frac{\sigma^{2} \varepsilon}{\lambda_{j}}\right)+\frac{1}{2} d^{2} \sigma^{2} \varepsilon^{2}+2 \frac{\sigma^{2} \varepsilon}{\lambda_{j}} \\
& <3 \sigma^{2} \varepsilon
\end{aligned}
$$

for $\varepsilon$ sufficiently small, e.g. $2 d^{2} \varepsilon \leqq 1$. The above bound and (3.2) imply also (3.3) as in (2.18), (2.19). From the fourth condition in (2.15) we get

$$
\left|\psi_{j}(y)-h\right| \leqq\left|\psi_{j}(y)-\left(Q_{j} \phi_{j}\right)(y)\right|+\left(Q_{j}\left|\phi_{j}-h\right|\right)(y)<\sigma^{2} \varepsilon+\frac{\sigma \varepsilon}{\sqrt{v_{j}}} \leqq 2 \frac{\sigma \varepsilon}{\sqrt{v_{j}}} .
$$

This inequality squared yields

$$
2\left(\left|\psi_{j}(y)\right||h|-\psi_{j}(y) \cdot h\right) \leqq|h|^{2}+\psi_{j}^{2}(y)-2 \psi_{j}(y) \cdot h<4 \frac{\sigma^{2} \varepsilon^{2}}{v_{j}},
$$

hence

$$
\left|v_{j}\left(1-\left(\psi_{j}(y)\right)_{0} \cdot h\right)\right|<2 \frac{\sigma^{2} \varepsilon^{2}}{\left|\psi_{j}(y)\right|}+v_{j}|1-| h||<2 \frac{\sigma^{2} \varepsilon^{2}}{1-3 \varepsilon}+\sigma^{2} \varepsilon^{2} \leqq 4 \sigma^{2} \varepsilon^{2}
$$

for $\varepsilon$ sufficiently small, e.g. $9 \varepsilon \leqq 1$, where $(\psi)_{0}=\frac{\psi}{|\psi|}$. The inequalities (3.1), (3.3), (3.5), (3.6) describe basic properties of configurations $\left(\psi_{j}, h\right)$ from the real space $\Xi_{j}(\sigma, \varepsilon)$. Consider now configurations $\left(\psi_{j}+\psi_{j}^{\prime}, h+h^{\prime}\right)$ from the complex space $\Xi_{j}^{c}(\sigma, \varepsilon)$. For $\psi_{j}^{\prime}$ we obtain

$$
\begin{gathered}
\left|\psi_{j}^{\prime}(y)\right| \leqq\left|\psi_{j}^{\prime}(y)-\left(Q_{j} \delta \phi_{j}\right)(y)\right|+\left(Q_{j}\left|\delta \phi_{j}\right|\right)(y)<\sigma^{2} \varepsilon+\varepsilon \\
\mid\left(\partial^{1} \psi^{\prime}(b) \mid<3 \sigma \varepsilon\right.
\end{gathered}
$$

the last inequality is obtained in the same way as (3.1). To the expression $\left(\psi_{j}(y)+\psi_{j}^{\prime}(y)\right) \cdot\left(\partial^{1}\left(\psi_{j}+\psi_{j}^{\prime}\right)\right)\left(\left\langle y, y^{\prime}\right\rangle\right)$ we apply the same transformation as in (3.2) and we obtain the bound

$$
\left|\left(\psi_{j}(y)+\psi_{j}^{\prime}(y)\right) \cdot\left(\partial^{1}\left(\psi_{j}+\psi_{j}^{\prime}\right)\right)\left(\left\langle y, y^{\prime}\right\rangle\right)\right|<7 \sigma^{2} \varepsilon
$$

for $\varepsilon$ sufficiently small. To the expression $\left(\psi_{j}(y)+\psi_{j}^{\prime}(y)\right)^{2}-\psi_{j}^{2}(y)$ we apply the transformation (3.4), and we obtain the bound

$$
\left|\left(\psi_{j}(y)+\psi_{j}^{\prime}(y)\right)^{2}-\psi_{j}^{2}(y)\right|<3 \sigma^{2} \varepsilon
$$

for $\varepsilon$ small, e.g. $6 d^{2} \varepsilon \leqq 1$. Finally we have

$$
\begin{aligned}
\left|\psi_{j}(y) \cdot h^{\prime}(y)\right| & \leqq\left|\left(\psi_{j}(y)-\left(Q_{j} \phi_{j}\right)(y)\right) \cdot h^{\prime}(y)\right|+\left|\left(Q_{j}\left(\phi_{j} \cdot h^{\prime}\right)\right)(y)\right| \\
& <\frac{\sigma^{4} \varepsilon^{2}}{v_{j}}+\frac{\sigma^{2} \varepsilon^{2}}{v_{j}} \leqq 2 \frac{\sigma^{2} \varepsilon^{2}}{v_{j}} .
\end{aligned}
$$

In the future we will have to estimate the expression with the unit vector $\left(\psi_{j}(y)\right)_{0}$. We have

$$
\left|\left(\psi_{j}(y)\right)_{0} \cdot h^{\prime}(y)\right|<\frac{2}{\left|\psi_{j}(y)\right|} \frac{\sigma^{2} \varepsilon^{2}}{v_{j}}<\frac{2}{1-3 \varepsilon} \frac{\sigma^{2} \varepsilon^{2}}{v_{j}}<3 \frac{\sigma^{2} \varepsilon^{2}}{v_{j}} .
$$


The above inequalities capture all important properties of the considered configurations, and they suggest the following definitions of new spaces:

$$
\begin{gathered}
\Psi_{j}(\sigma, \varepsilon)=\left\{\left(\psi_{j}, h\right):\left|\partial^{1} \psi_{j}\right|<\sigma \varepsilon,\left.|| \psi_{j}\right|^{2}-1 \mid<\sigma^{2} \varepsilon,\right. \\
\left.\left|v_{j}\left(1-\left(\psi_{j}\right)_{0} \cdot h\right)\right|<\sigma^{2} \varepsilon^{2},\left|v_{j}\left(h^{2}-1\right)\right|<\sigma^{2} \varepsilon^{2}\right\}, \quad\left(\psi_{j}\right)_{0}=\frac{\psi_{j}}{\left|\psi_{j}\right|}, \\
\Psi_{j}^{c}(\sigma, \varepsilon)=\left\{\left(\psi_{j}+\psi_{j}^{\prime}, h+h^{\prime}\right):\left(\psi_{j}, h\right) \in \Psi_{j}(\sigma, \varepsilon),\left|\psi_{j}^{\prime}\right|<\varepsilon+\sigma^{2} \varepsilon,\right. \\
\left.\left|\partial^{1} \psi_{j}^{\prime}\right|<\sigma \varepsilon,\left|\left(\psi_{j}+\psi_{j}^{\prime}\right)^{2}-\psi_{j}^{2}\right|<\sigma^{2} \varepsilon,\left|v_{j} h^{\prime}\right|<\sigma^{2} \varepsilon,\left|v_{j}\left(\psi_{j}\right)_{0} \cdot h^{\prime}\right|<\sigma^{2} \varepsilon^{2}\right\} .
\end{gathered}
$$

These spaces depend on $v_{j}$. If we want to indicate it explicitly, or if we want to consider more generally a parameter $v$, we will write $\Psi_{j}(\sigma, \varepsilon ; v), \Psi_{j}^{c}(\sigma, \varepsilon ; \nu)$. The above definitions describe the most important properties of the considered configurations. In particular the expressions occurring in the definition (3.13) are the basic scaling, or rather pseudo-scaling expressions in our renormalization group approach. We will expand effective actions in terms of these expressions. Notice that, as in the case of the definition (2.16), we may replace the conditions in (3.14) by the conditions

$$
\begin{gathered}
\left|\psi_{j}^{\prime}\right|<\varepsilon+\sigma^{2} \varepsilon, \quad\left|\partial^{1}\left(\psi_{j}+\psi_{j}^{\prime}\right)\right|<2 \sigma \varepsilon, \quad\left|\left(\psi_{j}+\psi_{j}^{\prime}\right)^{2}-1\right|<2 \sigma^{2} \varepsilon, \quad\left|v_{j} h^{\prime}\right|<\sigma^{2} \varepsilon, \\
\left|v_{j}\left(\psi_{j}\right)_{0} \cdot h^{\prime}\right|<\sigma^{2} \varepsilon^{2} .
\end{gathered}
$$

The results of the transformations and bounds (3.1)-(3.12) imply the following lemma

\section{Lemma 3.1.}

$$
\Xi_{j}(\sigma, \varepsilon) \subset \Psi_{j}(3 \sigma, \varepsilon), \quad \Xi_{j}^{c}(\sigma, \varepsilon) \subset \Psi_{j}^{c}(3 \sigma, \varepsilon)
$$

for \& sufficiently small.

The spaces $\Psi_{j}^{c}(\sigma, \varepsilon)$ are crucial for the renormalization analysis, but they cannot be used as a basis of our inductive construction, in particular we cannot use them as analyticity domains of the functions $\mathscr{E}^{(j)}$, because the functions $\psi_{k}^{(j)}$ do not establish generally any simple relations between these spaces. We mean here relations of the type described in Lemma 2.1, which are necessary for the inductive construction. They are the main reason for using the spaces $\Xi_{j}^{c}$ instead of $\Psi_{j}^{c}$. Actually we have to use both classes of spaces for reasons which will be clarified in the future. Now let us describe briefly the way we use these spaces. The most important conclusion of Lemma 2.1 can be written as the statement

$$
\psi_{k}^{(j)}: \Xi_{k}^{c}\left(1, \varepsilon_{k}\right) \rightarrow \Xi_{j}^{c}\left(L^{j} \eta, \varepsilon_{k}\right) \subset \Xi_{j}^{c}\left(1, \varepsilon_{j}\right), \quad j \leqq k .
$$

It implies that the effective action $\mathscr{A}_{k}$ is an analytic function defined on $\Xi_{k}^{c}\left(1, \varepsilon_{k}\right)$. To perform various operations connected with the renormalization analysis we have to interpolate between the last two spaces in (3.17) using proper spaces $\Psi_{j}^{c}(\sigma, \varepsilon)$. By Lemma 3.1 we have $\Xi_{j}^{c}\left(L^{j} \eta, \varepsilon_{k}\right) \subset \Psi_{j}^{c}\left(C_{0} L^{j} \eta, \varepsilon_{k}\right)$ if $C_{0} \geqq 3$, and the question is whether the last space is contained in $\Xi_{j}^{c}\left(1, \varepsilon_{j}\right)$. This holds for $k-j$ sufficiently large, or for $C_{0} L^{j} \eta$ sufficiently small. It is a statement about bounds satisfied by the function $\phi_{j}$ considered on the space $\Psi_{j}^{c}\left(C_{0} L^{j} \eta, \varepsilon_{k}\right)$. These bounds are formulated in definitions (2.15), (2.16),(2.20),(2.21) with $\sigma=1, \varepsilon=\varepsilon_{j}$. Such bounds hold with milder assumptions on configurations $\left(\psi_{j}+\psi_{j}^{\prime}, h+h^{\prime}\right)$. Let us introduce another class of spaces, simpler than the spaces $\Psi_{j}^{c}$. We do not need the second condition in 
(3.13) and the third condition in (3.14), we also bound $\sigma^{2}$ by $\sigma$ in the third condition in (3.13), and in the first and fourth conditions in (3.14). A reason for this is that the bounds for $\phi_{j}$ usually involve most of the conditions in (3.13), (3.14), in particular the conditions on derivatives, so the constant $\sigma \varepsilon$ is the best we can get in those bounds. For simplicity let us denote $\delta=\sigma \varepsilon$, so $\delta \leqq \varepsilon$, and define

$$
\begin{gathered}
\widetilde{\Psi}_{j}(\delta)=\left\{\left(\psi_{j}, h\right):\left|\partial^{1} \psi_{j}\right|<\delta,\left.\quad|| \psi_{j}\right|^{2}-1|<\delta, \quad| v_{j}\left(1-\left(\psi_{j}\right)_{0} \cdot h\right) \mid<\delta^{2},\right. \\
\left.\left|v_{j}\left(h^{2}-1\right)\right|<\delta^{2}\right\} \\
\widetilde{\Psi}_{j}^{c}(\delta, \varepsilon)=\left\{\left(\psi_{j}+\psi_{j}^{\prime}, h+h^{\prime}\right):\left(\psi_{j}, h\right) \in \widetilde{\Psi}_{j}(\delta),\left|\psi_{j}^{\prime}\right|<\varepsilon+\delta,\right. \\
\left.\left|\partial^{1} \psi_{j}^{\prime}\right|<\delta,\left|\left(\psi_{j}+\psi_{j}^{\prime}\right)^{2}-\psi_{j}^{2}\right|<\delta,\left|v_{j} h^{\prime}\right|<\frac{\delta^{2}}{\varepsilon},\left|v_{j}\left(\psi_{j}\right)_{0} \cdot h^{\prime}\right|<\delta^{2}\right\} .
\end{gathered}
$$

The above spaces will be used in the case $\sigma=C_{0} L^{j} \eta, \varepsilon=\varepsilon_{k}$, and $v_{j}=v_{k}\left(L^{j} \eta\right)^{2}$, hence $v_{j} \leqq\left(L^{j} \eta\right)^{2} \leqq \sigma^{2}=\left(\frac{\delta}{\varepsilon}\right)^{2}$. We assume generally that $v_{j} \leqq\left(\frac{\delta}{\varepsilon}\right)^{2}$. Obviously we have

$$
\Psi_{j}(\sigma, \varepsilon) \subset \tilde{\Psi}_{j}(\delta), \quad \tilde{\Psi}_{j}^{c}(\sigma, \varepsilon) \subset \tilde{\Psi}_{j}^{c}(\delta, \varepsilon) \text { if } \sigma \varepsilon \leqq \delta .
$$

The space $\widetilde{\Psi}_{j}(\delta)$ is defined by simplest and most natural smallness conditions on basic quantities connected with the action (1.5). The definition of the space $\widetilde{\Psi}_{j}^{c}(\delta, \varepsilon)$ extends these conditions to complex variables, except that the variable $\psi_{j}^{\prime}$ is treated separately in a special way. This is because it does not scale, or rather it does not scale with a negative power of $L$ as the other quantities, so bounds for functions involving $\psi_{j}^{\prime}$, in particular bounds for $\delta \phi_{j}\left(\psi_{j}^{\prime}\right)$, have to be treated carefully. From Lemma 3.1 and (3.20) we obtain the inclusion $\Xi_{j}^{c}(\sigma, \varepsilon) \subset \widetilde{\Psi}_{j}^{c}(3 \sigma \varepsilon, \varepsilon)$. One of the fundamental results of the next paper can be formulated in the following way: there exists a constant $K_{1}>1$ depending on $d$ only, such that

$$
\tilde{\Psi}_{j}^{c}(\delta, \varepsilon) \subset \Xi_{j}^{c}(1, \tilde{\varepsilon}) \text { if } \varepsilon+K_{1} \delta \leqq \tilde{\varepsilon}
$$

for $\varepsilon, \tilde{\varepsilon}$ sufficiently small. If not for the conditions on $\psi_{j}^{\prime}, \delta \phi_{j}\left(\psi_{j}^{\prime}\right)$ we would have $K_{1} \delta \leqq \tilde{\varepsilon}$ in the above statement. Unfortunately these conditions will cause some complications in the future. From the above statements we obtain the lemma

\section{Lemma 3.2}

$$
\begin{gathered}
\psi_{k}^{(j)}: \Xi_{k}^{c}\left(1, \varepsilon_{k}\right) \rightarrow \Xi_{j}^{c}\left(L^{j} \eta, \varepsilon_{k}\right) \subset \Psi_{j}^{c}\left(C_{0} L^{j} \eta, \varepsilon_{k}\right) \\
\subset \Psi_{j}^{c}\left(\sigma, \varepsilon_{k}\right) \subset \widetilde{\Psi}_{j}^{c}\left(\delta, \varepsilon_{k}\right) \subset \widetilde{\Psi}_{j}^{c}\left(\delta_{0}, \varepsilon_{0}\right) \subset \Xi_{j}^{c}\left(1, \varepsilon_{j}\right) \\
\text { if } C_{0} \geqq 3, C_{0} L^{j} \eta \leqq \sigma, \sigma \varepsilon_{k} \leqq \delta, \delta \leqq \delta_{0}, \varepsilon_{k} \leqq \varepsilon_{0}, \frac{\delta}{\varepsilon_{k}} \leqq \frac{\delta_{0}}{\varepsilon_{0}}, \varepsilon_{0}+K_{1} \delta_{0} \leqq \varepsilon_{j}
\end{gathered}
$$

This lemma establishes a scheme of applications of the above spaces. We consider a function $\mathscr{E}^{(j)}$ on the space $\widetilde{\Psi}_{j}^{c}\left(\delta_{0}, \varepsilon_{0}\right)$ with properly chosen $\delta_{0}, \varepsilon_{0}$ and we perform all operations on this space, e.g. applications of Taylor fomula. To bound expressions obtained by such operations we use the space $\Psi_{j}^{c}\left(\sigma, \varepsilon_{k}\right)$ with a properly chosen $\sigma$, e.g. $\sigma=C_{0} L^{j} \eta$. This scheme will be applied usually with $\varepsilon_{0}=\varepsilon_{j+1}$.

Now we describe the main steps of the renormalization analysis and give some additional explanations for definitions introduced before. The action $\mathscr{E}_{k}$ has the representations $(\mathrm{H} .2),\left(\right.$ H.5). Take a term $\mathscr{E}^{(j)}\left(y, X ; \psi_{k}^{(j)}, v_{j} h\right)$ in the corresponding 
sum. It is a function of $\psi_{k}^{(j)}$ and we want to expand it around a constant configuration equal to a value $\psi_{k}^{(j)}(y)$. There are several problems connected with such an expansion. The function $\mathscr{E}^{(j)}\left(y, X ; \psi_{j}, v_{j} h\right)$ has an analytic extension defined on $\Xi_{j}^{c}\left(1, \varepsilon_{j}\right)$. A problem with this space is that if we take a configuration $\psi_{j}$ from it, then a constant configuration equal to a value $\psi_{j}(y)$ at a point $y \in T^{(j)}$ does not necessarily belong to the space. To overcome this problem we consider the function on a smaller space $\tilde{\Psi}_{j}^{c}(\delta, \varepsilon)$ with $\delta, \varepsilon$ satisfying the condition in Lemma 3.2. This space obviously has the desired property, namely if a configuration $\psi_{j}$ belongs to it, then constant configurations equal to values $\psi_{j}(y)$ at points of $T^{(j)}$ belong to the space also. This is the main reason for introducing these spaces. The spaces $\Psi_{j}^{c}(\sigma, \varepsilon)$ have this property also, and they are introduced to describe precisely pseudoscaling properties of corresponding local functions of $\psi_{j}, h$. Consider now the above mentioned expansion around the constant configuration. A problem with this expansion is that a remainder depends on configurations $\psi_{j, t}=\psi_{j}(y)+t \delta \psi_{j}$, $\delta \psi_{j}(x)=\psi_{j}(x)-\psi_{j}(y), 0 \leqq t \leqq 1$. These configurations generally do not belong to the space $\widetilde{\Psi}_{j}^{c}(\delta, \varepsilon)$ for $\psi_{j}$ from this space, in particular the conditions defining the space are not satisfied usually for points $x$ sufficiently far away from the point $y$. This forces us to restrict further the space, or to decrease the constants $\delta, \varepsilon$, and to impose a restriction on $X$, more precisely on $d_{j}(X)$. We consider the function $\mathscr{E}^{(j)}$ on configurations $\psi_{k}^{(j)}$, so we have to make sure that these configurations belong to the space $\widetilde{\Psi}_{j}^{c}(\delta, \varepsilon)$. By Lemma 3.2 this is true if $k-j$ is sufficiently large, e.g. $k-j \geqq n_{1}$, or $j \leqq k-n_{1}$. Precise conditions on $n_{1}$ will be obtained later. Now a basis of the renormalization analysis and the proof of the uniform bound on $\mathscr{E}_{k}$ is the following formula:

$$
\begin{aligned}
\mathscr{E}_{k}\left(\psi_{k}, h\right)= & \sum_{j=1}^{k-n_{1}} \sum_{y \in T^{(j)}} \mathscr{V}^{(j)}\left(\psi_{k}^{(j)}(y), v_{j} h\right) \\
& +\sum_{j=1}^{k-n_{1}} \sum_{y \in T^{(j)}} \sum_{X \in \mathscr{\mathscr { Q }} ;: y \in X}\left[\mathscr{E}^{(j)}\left(y, X ; \psi_{k}^{(j)}, v_{j} h\right)-\mathscr{E}^{(j)}\left(y, X ; \psi_{k}^{(j)}(y), v_{j} h\right)\right] \\
& +\sum_{j=k}^{k} \sum_{k=n_{1}+1} \mathscr{E}^{(j)}\left(y ; \psi_{k}^{(j)}, v_{j} h\right)
\end{aligned}
$$

where we have used the definition (2.25) of the effective potentials $\mathscr{V}^{(j)}$. A dependence on $\psi_{k}$ above is through the functions $\psi_{k}^{(j)}$. In the rest of this subsection we analyze successively terms of three sums above.

Consider a term of the first sum. By Lemma 3.2 the values $\psi_{k}^{(j)}(y)$ are in a domain $\Psi_{j \text {,const }}^{c}\left(\sigma, \varepsilon_{k}\right), \sigma \geqq C_{0} L^{j} \eta$, which is defined as a domain in $\mathbf{C}^{N} \times \mathbf{C}^{N}$ determining constant configurations in $\Psi_{j}^{c}\left(\sigma, \varepsilon_{k}\right)$. It is defined by (3.13), (3.14), but with conditions on derivatives dropped out. We would like to obtain bounds on the effective potential $\mathscr{V}^{(j)}$ considered on this domain. This potential is defined and analytic on a larger domain, certainly on $\widetilde{\Psi}_{j \text {,const }}^{c}\left(\delta_{0}, \varepsilon_{0}\right)$, where $\delta_{0}, \varepsilon_{0}$ satisfy the conditions in Lemma 3.2. Let us analyze these conditions in more detail. Obviously they can be satisfied only if $k-j$ is sufficiently large, for example we conclude that $K_{1} C_{0} L^{j} \eta \varepsilon_{k}<\varepsilon_{j}$. We can take $\varepsilon_{0}=\varepsilon_{j+1}$ above, and then the crucial condition is $\varepsilon_{j+1}+K_{1} \delta_{0} \leqq \varepsilon_{j}$. We have $\varepsilon_{j}-\varepsilon_{j+1}=\varepsilon_{j+1}\left(L^{\frac{d-2}{2}-\gamma}-1\right) \geqq \varepsilon_{j+1} \log L\left(\frac{d-2}{2}-\gamma\right)$. Let us denote for simplicity $\frac{d-2}{2}-\gamma=\alpha$, so the condition is satisfied if $K_{1} \delta_{0} \leqq \alpha \varepsilon_{j+1}$. We take $\delta_{0}=\sigma_{0} \varepsilon_{j+1}$, hence the condition is $K_{1} \sigma_{0} \leqq \alpha$. For $\sigma_{0}=C_{0} L^{j} \eta$ we obtain a restriction on $k-j$ of the form $C_{0} K_{1} L^{j-\bar{k}} \leqq \alpha$, or 
$L^{k-j} \geqq C_{0} K_{1} \frac{1}{\alpha}$. We choose $n_{1} \geqq$ the smallest integer satisfying the last inequality. With this assumption we have the inclusions

$$
\psi_{k}^{(j)}(y): \Xi_{k}^{c}\left(1, \varepsilon_{k}\right) \rightarrow \Psi_{j, \text { const }}^{c}\left(\sigma, \varepsilon_{k}\right) \subset \tilde{\Psi}_{j, \text { const }}^{c}\left(\delta, \varepsilon_{k}\right) \subset \tilde{\Psi}_{j, \text { const }}^{c}\left(\delta_{1}, \varepsilon_{j+1}\right),
$$

where $\sigma=C_{0} L^{j} \eta, \delta=\sigma \varepsilon_{k}, \delta_{1}=\sigma_{1} \varepsilon_{j+1}, \sigma_{1}=K_{1}^{-1} \alpha$. The effective potential $\mathscr{V}^{(j)}$ is analytic on the last domain above.

The most important property of the effective potential is the invariance (H.4) with respect to orthogonal transformations. We study consequences of this symmetry quite generally, so let us drop the index $j$. Consider a function $\mathscr{V}(\psi, v h)$ of real vectors $(\psi, h)$ in a domain invariant with respect to the group $O(N)$, e.g. in some $\tilde{\Psi}_{\text {const }}^{c}\left(\delta_{0}\right)$. Take at first $R_{1}$ transforming a vector $\psi$ into $|\psi| e_{1}, R_{1} \psi=|\psi| e_{1}$, hence $R_{1}^{-1} e_{1}=\psi_{0}$, and $\mathscr{V}(\psi, v h)=\mathscr{V}\left(|\psi| e_{1}, v R_{1} h\right)$. We write $R_{1} h=$ $\left(R_{1} h\right)_{1} e_{1}+\left(R_{1} h\right)^{\prime}$, where $e_{1} \cdot\left(R_{1} h\right)^{\prime}=0$, and we take $R_{2}$ such that $R_{2} e_{1}=e_{1}$, $R_{2}\left(R_{1} h\right)^{\prime}=\left|\left(R_{1} h\right)^{\prime}\right| e_{2}$. We have $\left(R_{1} h\right)_{1}=e_{1} \cdot R_{1} h=R_{1}^{-1} e_{1} \cdot h=\psi_{0} \cdot h$, and $\left|R_{1} h\right|$ $=|h|$, hence $\left|\left(R_{1} h\right)^{\prime}\right|^{2}=|h|^{2}-\left(R_{1} h\right)_{1}^{2}=|h|^{2}-\left(\psi_{0} \cdot h\right)^{2}$. From the above equalities we obtain

$$
\mathscr{V}(\psi, v h)=\mathscr{V}\left(|\psi| e_{1}, v\left(\psi_{0} \cdot h\right) e_{1}+v \sqrt{|h|^{2}-\left(\psi_{0} \cdot h\right)^{2}} e_{2}\right) .
$$

Consider the function $\mathscr{V}\left(u e_{1}, v v e_{1}+v w e_{2}\right)$. It is defined for $u$ close to 1 and $(v, w)$ in a neighborhood of the unit circle in $\mathbf{R}^{2}$, and it has an analytic extension to a function of $u+u^{\prime}, v+v^{\prime}, w+w^{\prime}$, where $u^{\prime}, v^{\prime}, w^{\prime}$ are complex numbers in a neighborhood of 0 . More precisely we consider the function $\mathscr{V}\left(\left(u+u^{\prime}\right) e_{1}\right.$, $\left.v\left(v+v^{\prime}\right) e_{1}+v\left(w+w^{\prime}\right) e_{2}\right)$, which is an analytic function of $u+u^{\prime}, v+v^{\prime}, w+w^{\prime}$, defined on the domain

$$
\begin{aligned}
& u, v, w \in \mathbf{R}, u>0,\left|u^{2}-1\right|<\delta_{0},\left|v\left(v^{2}+w^{2}-1\right)\right|<\delta_{0}^{2},|v(1-v)|<\delta_{0}^{2}, \\
& u^{\prime}, v^{\prime}, w^{\prime} \in \mathbf{C},\left|u^{\prime}\right|<\varepsilon_{0}+\delta_{0},\left|\left(u+u^{\prime}\right)^{2}-u^{2}\right|<\delta_{0},\left|v v^{\prime}\right|<\delta_{0}^{2},\left|v w^{\prime}\right|<\frac{1}{\sqrt{2}} \frac{\delta_{0}^{2}}{\varepsilon_{0}} .
\end{aligned}
$$

For the $\mathrm{j}^{\text {th }}$ potential we shall take $\delta_{0}=\delta_{1}, \varepsilon_{0}=\varepsilon_{j+1}$. This function is invariant with respect to rotations of the plane spanned by $e_{1}, e_{2}$ which leave the vector $e_{1}$ invariant. It means simply that it is an even function of $w+w^{\prime}$. We define

$$
f\left(u+u^{\prime}, v\left(v+v^{\prime}\right), v^{2}\left(w+w^{\prime}\right)^{2}\right)=\mathscr{V}\left(\left(u+u^{\prime}\right) e_{1}, v\left(v+v^{\prime}\right) e_{1}+v\left(w+w^{\prime}\right) e_{2}\right) .
$$

For simplicity we write it as $f\left(u, v v, v^{2} w^{2}\right)$, and the complex numbers $u, v, w$ belong to the domain described by (3.26). From the above definition and the equality (3.25) we obtain a representation

$$
\mathscr{V}(\psi, v h)=f\left(|\psi|, v\left(\psi_{0} \cdot h\right), v^{2}\left(|h|^{2}-\left(\psi_{0} \cdot h\right)^{2}\right)\right) .
$$

This is a fundamental representation of the effective potentials, it is formulated in terms of invariants of the orthogonal group $O(N)$, and it is a basis of the renormalization analysis. From the restrictions defining $\widetilde{\Psi}_{\text {const }}\left(\delta_{0}\right)$ we see that the expressions $|\psi|-1, v\left(1-\psi_{0} \cdot h\right)$ are small, and they are also the basic pseudo-scaling quantities, as it has been mentioned before, so it is natural to expand the effective potentials with respect to those expressions. This will give us an expansion into relevant, marginal and irrelevant quantities. To construct such an expansion we 
have to expand the function $f\left(u, v u, v^{2} w^{2}\right)$ around the point $u=1, v=1, w=0$. Obviously this point belongs to the domain (3.26) for any $\delta_{0}$ and $\varepsilon_{0}$. To study this expansion we restrict the function to a polydisc with a center at this point. It is easy to see that the polydisc in $\mathbf{C}^{3}$ defined by

$$
|u-1|<\frac{1}{3} \delta_{0}, \quad|v-1|<\frac{\delta_{0}^{2}}{v}, \quad|w|<\frac{1}{\sqrt{2}} \frac{\delta_{0}^{2}}{v \varepsilon_{0}},
$$

is contained in the domain (3.26). The function $f\left(u, v v, v^{2} w^{2}\right)$ is analytic on it and bounded by $E_{0} K_{0}$, hence derivatives of this function satisfy the following bounds:

$$
\left|\frac{\partial^{\alpha+\beta+\gamma}}{(\partial u)^{\alpha}(\partial v v)^{\beta}\left(\partial\left(v^{2} w^{2}\right)\right)^{\gamma}} f\left(u, v v, v^{2} w^{2}\right)\right| \leqq \frac{8 \alpha ! \beta ! \gamma ! 6^{\alpha} 2^{\beta} 4^{\gamma} E_{0} K_{0} \varepsilon_{0}^{2 \gamma}}{\delta_{0}^{\alpha+2 \beta+4 \gamma}}
$$

on the polydisc

$$
|u-1|<\frac{1}{6} \delta_{0}, \quad|v-1|<\frac{1}{2} \frac{\delta_{0}^{2}}{v}, \quad|w|<\frac{1}{2} \frac{\delta_{0}^{2}}{v \varepsilon_{0}} .
$$

Of course only the powers of $\delta_{0}, \varepsilon_{0}$, and the factor $E_{0} K_{0}$ are important in the above bounds.

We can write now a Taylor expansion of the function $f$ to any order, and we can write bounds on its terms based on the bounds (3.30). Having in mind applications to the effective potentials we write the following expansion:

$$
\begin{aligned}
f\left(u, v v, v^{2} w^{2}\right)= & f(1, v, 0)+\left(\frac{\partial}{\partial u} f\right)(1, v, 0)(u-1)+\left(\frac{\partial}{\partial v v} f\right)(1, v, 0) v(v-1) \\
& +f_{2,0,0}(u, v, 0)(u-1)^{2}+f_{1,1,0}(u, v, 0)(u-1) v(v-1) \\
& +f_{0,2,0}(u, v v, 0)(v(v-1))^{2}+f_{0,0,1}\left(u, v u, v^{2} w^{2}\right) v^{2} w^{2}
\end{aligned}
$$

where the functions $f_{\alpha, \beta, \gamma}$ are expressed in terms of the corresponding derivatives of the orders $\alpha, \beta, \gamma$ by the integral representation of remainders in the Taylor formula. From this representation and the bounds (3.30) we obtain

$$
\begin{aligned}
& \left|f\left(u, v v_{1}, v^{2} w^{2}\right)-f(1, v, 0)-\left(\frac{\partial}{\partial u} f\right)(1, v, 0)(u-1)-\left(\frac{\partial}{\partial v v} f\right)(1, v, 0) v(v-1)\right| \\
& \leqq 8 E_{0} K_{0}\left[\left(\frac{6}{\delta_{0}}|u-1|\right)^{2}+\frac{6}{\delta_{0}}|u-1| \cdot \frac{2}{\delta_{0}^{2}}|v(v-1)|\right. \\
& \left.+\left(\frac{2}{\delta_{0}^{2}}|v(v-1)|\right)^{2}+\frac{4 \varepsilon_{0}^{2}}{\delta_{0}^{4}}\left|v^{2} w^{2}\right|\right]
\end{aligned}
$$

on the polydisc (3.31).

Now we apply the results of the above general analysis to the effective potentials $\mathscr{V}^{(j)}\left(\psi, v_{j} h\right)$ for $j \leqq k-n_{1}$. We have the representations (3.28) with functions $f^{(j)}$ which are analytic on the domain (3.26), hence on (3.29) with $\delta_{0}=\delta_{1}$ and $\varepsilon_{0}=\varepsilon_{j+1}$. They have the expansions (3.32) and satisfy the bounds (3.33) on the polydisc (3.31). We formulate the hypothesis (H.6) in terms of the corresponding functions $f^{(j)}$. We have

$$
f^{(j)}\left(u, v_{j} v, 0\right)=\mathscr{V}^{(j)}\left(u e_{1}, v_{j} v e_{1}\right)=\mathscr{E}^{(j)}\left(y ; u e_{1}, v_{j} v e_{1}\right)
$$


for any $y \in T^{(j)}$. Using these equalities we can rewrite (H.6) in the form

$$
f^{(j)}\left(1, v_{j}, 0\right)=0,\left(\frac{\partial}{\partial u} f^{(j)}\right)\left(1, v_{j}, 0\right)=0,\left(\frac{\partial}{\partial v_{j} v} f^{(j)}\right)\left(1, v_{j}, 0\right)=0 .
$$

From this and the bound (3.33) we obtain immediately the bounds

$$
\begin{aligned}
\left|f^{(j)}\left(u, v_{j} v, v_{j}^{2} w^{2}\right)\right| \leqq & 8 E_{0} K_{0}\left[\frac{3}{2}\left(\frac{6}{\delta_{1}}|u-1|\right)^{2}+\frac{3}{2}\left(\frac{2}{\delta_{1}^{2}}\left|v_{j}(v-1)\right|\right)^{2}\right. \\
& \left.+\frac{4 \varepsilon_{j+1}^{2}}{\delta_{1}^{4}}\left|v_{j}^{2} w^{2}\right|\right]
\end{aligned}
$$

on the polydisc (3.31) with $\delta_{0}=\delta_{1}, \varepsilon_{0}=\varepsilon_{j+1}, v=v_{j}$.

We have obtained the representation (3.28) of the effective potential for real vectors $\psi, h$. We can construct an analytic extension of the potential by using the analyticity of the function $f$ and taking analytic extensions of the expressions inside the function. We introduce the following three functions of $\psi+\psi^{\prime}, h+h^{\prime}$ :

$$
\begin{aligned}
U & =\sqrt{\left(\psi+\psi^{\prime}\right)^{2}}, V=\left(\psi+\psi^{\prime}\right)_{0} \cdot\left(h+h^{\prime}\right), \\
W^{2} & =\left(h+h^{\prime}\right)^{2}-V^{2}=\left(h+h^{\prime}\right)^{2}-\left(\left(\psi+\psi^{\prime}\right)_{0} \cdot\left(h+h^{\prime}\right)\right)^{2} .
\end{aligned}
$$

The functions $U, V, W^{2}$ are natural analytic extensions of $|\psi|, \psi_{0} \cdot h,|h|^{2}$ $-\left(\psi_{0} \cdot h\right)^{2}$. We have to obtain bounds for these functions considered on the domain $\Psi_{\text {const }}^{c}(\sigma, \varepsilon)$. We have

$$
\begin{aligned}
U-1= & \left(\sqrt{1+\left(\psi^{2}-1\right)+\left(\left(\psi+\psi^{\prime}\right)^{2}-\psi^{2}\right)}+1\right)^{-1} \\
& \times\left[\left(\psi^{2}-1\right)+\left(\left(\psi+\psi^{\prime}\right)^{2}-\psi^{2}\right)\right],
\end{aligned}
$$

hence

$$
|U-1|<2 \sigma^{2} \varepsilon,
$$

assuming $\varepsilon$ sufficiently small. Next

$$
\begin{aligned}
V-1= & \left(\psi+\psi^{\prime}\right)_{0} \cdot\left(h+h^{\prime}\right)-1=\left(\psi_{0} \cdot h-1\right)+\frac{1}{|\psi|}\left(I-\psi_{0} \otimes \psi_{0}\right) \psi^{\prime} \cdot h \\
& -\int_{0}^{1} d t(1-t) \frac{1}{\left(\psi+t \psi^{\prime}\right)^{2}}\left\{2 \left(\left[I-\left(\psi+t \psi^{\prime}\right)_{0}\right.\right.\right. \\
& \left.\left.\otimes\left(\psi+t \psi^{\prime}\right)_{0}\right] \psi^{\prime} \cdot h\right)\left(\left(\psi+t \psi^{\prime}\right)_{0} \cdot \psi^{\prime}\right) \\
& \left.\left.+\left(\psi^{\prime} \cdot\left[I-\left(\psi+t \psi^{\prime}\right)_{0} \otimes\left(\psi+t \psi^{\prime}\right)_{0}\right] \psi^{\prime}\right)\left(\left(\psi+t \psi^{\prime}\right)_{0} \cdot h\right)\right)\right\}+\psi_{0} \cdot h^{\prime} \\
& +\int_{0}^{1} d t \frac{1}{\sqrt{\left(\psi+t \psi^{\prime}\right)^{2}}}\left[I-\left(\psi+t \psi^{\prime}\right)_{0} \otimes\left(\psi+t \psi^{\prime}\right)_{0}\right] \psi^{\prime} \cdot h^{\prime}
\end{aligned}
$$

and $\left(I-\psi_{0} \otimes \psi_{0}\right) \psi^{\prime} \cdot h=\psi^{\prime} \cdot\left(h-\psi_{0}\right)+\left(1-\psi_{0} \cdot h\right)\left(\psi^{\prime} \cdot \psi_{0}\right)$. 
These identities yield the estimate

$$
\begin{aligned}
|V-1|< & \frac{\sigma^{2} \varepsilon^{2}}{v}+\frac{2 \varepsilon}{1-\varepsilon}\left(\frac{\sqrt{3} \sigma \varepsilon}{\sqrt{v}}+\frac{\sigma^{2} \varepsilon^{2}}{v}\right)+8 \varepsilon^{2}\left(1+\frac{\sigma^{2} \varepsilon^{2}}{v}\right)+4 \varepsilon^{2}\left(1+\frac{\sigma^{2} \varepsilon^{2}}{v}\right) \\
& +\frac{\sigma^{2} \varepsilon^{2}}{v}+4 \varepsilon \frac{\sigma^{2} \varepsilon}{v}<22 \frac{\sigma^{2} \varepsilon^{2}}{v}
\end{aligned}
$$

where we have used the assumption $v \leqq \sigma^{2}$. Notice that the above estimate is not an optimal one, we have simply estimated all expressions close to 1 by 2 in each term. For the function $W^{2}$ we have

$$
\begin{aligned}
W^{2}= & \left(|h|^{2}-1 \mid\right)+2\left(\psi_{0} \cdot h\right) \psi_{0} \cdot h^{\prime}+2\left(I-\psi_{0} \otimes \psi_{0}\right) h \cdot h^{\prime} \\
& +\left(h^{\prime}\right)^{2}-2(V-1)-(V-1)^{2},
\end{aligned}
$$

hence

$$
\begin{aligned}
\left|W^{2}\right|< & \frac{\sigma^{2} \varepsilon^{2}}{v}+2\left(1+\frac{\sigma^{2} \varepsilon^{2}}{v}\right) \frac{\sigma^{2} \varepsilon^{2}}{v}+2 \frac{\sqrt{3} \sigma \varepsilon}{\sqrt{v}} \cdot \frac{\sigma^{2} \varepsilon}{v}+2\left(\frac{\sigma^{2} \varepsilon^{2}}{v}\right)^{2} \\
& +\frac{\sigma^{2} \varepsilon^{2}}{v^{2}}+44 \frac{\sigma^{2} \varepsilon^{2}}{v}+22 \frac{\sigma^{4} \varepsilon^{4}}{v^{2}}<56 \frac{\sigma^{4} \varepsilon^{2}}{v^{2}}
\end{aligned}
$$

for $\varepsilon$ sufficiently small. We conclude from the inequalities (3.39), (3.41), (3.43) that the following bounds hold:

$$
|U-1|<\frac{1}{6}(7 \sigma)^{2} \varepsilon,|V-1|<\frac{1}{2} \frac{(7 \sigma)^{2} \varepsilon^{2}}{v},\left|W^{2}\right|<\frac{1}{4} \frac{(7 \sigma)^{4} \varepsilon^{2}}{v^{2}} .
$$

We obtain the lemma

Lemma 3.3. The analytic functions $U, V, W^{2}$ map the domain $\Psi_{\text {const }}^{c}(\sigma, \varepsilon)$ into the polydisc (3.31) if $\varepsilon \leqq \varepsilon_{0}, 7 \sigma \varepsilon_{0} \leqq \delta_{0}$.

We apply the lemma in the case where $\varepsilon_{0}=\varepsilon_{j+1}, \delta_{0}=\delta_{1}=\sigma_{1} \varepsilon_{j+1}$, and then the condition $7 \sigma \varepsilon_{0} \leqq \delta_{0}$ means $7 \sigma \leqq \sigma_{1}$. We have found that $\sigma_{1}=K_{1}^{-1} \alpha$, hence it can be written as $7 K_{1} \frac{1}{\alpha} \sigma \leqq 1$. We have also $\sigma=C_{0} L^{j} \eta$, so the condition takes the form $7 C_{0} K_{1} \frac{1}{\alpha} L^{j} \eta \leqq 1$, or $L^{k-j} \geqq 7 C_{0} K_{1} \frac{1}{\alpha}$. It implies the previous one, which has the same form but with 7 replaced by 2 . We adjust $n_{1}$ properly so that the above condition is satisfied.

Let us consider the equality (3.28). The function $f$ is analytic on the polydisc (3.31), so the right-hand side of the equality has an analytic extension defined on the domain $\Psi_{\text {const }}^{c}\left(\sigma, \varepsilon_{0}\right)$, if $7 \sigma \varepsilon_{0} \leqq \delta_{0}$. This defines an analytic extension of the effective potential by the formula

$$
\mathscr{V}\left(\psi+\psi^{\prime}, v\left(h+h^{\prime}\right)\right)=f\left(U, v V, v^{2} W^{2}\right) .
$$

Consider now the effective potentials $\mathscr{V}^{(j)}$. They have analytic extensions given by the corresponding formulas (3.45), and defined on domains $\Psi_{\text {const }}^{c}(\sigma, \varepsilon)$ with $7 \sigma \leqq \sigma_{1}, \varepsilon \leqq \varepsilon_{j+1}$. We take $\varepsilon_{0}=\varepsilon_{j+1}, \delta_{0}=\delta_{1}=\sigma_{1} \varepsilon_{j+1}, v=v_{j}$ in definitions and 
bounds above. The equality (3.45) and the bounds (3.36), (3.44) imply

$$
\begin{aligned}
\left|\mathscr{V}^{(j)}\left(\psi+\psi^{\prime}, v_{j}\left(h+h^{\prime}\right)\right)\right| & <8 E_{0} K_{0}\left(\frac{3}{2} \frac{1}{\delta_{1}^{2}}(7 \sigma)^{4} \varepsilon^{2}+\frac{3}{2} \frac{1}{\delta_{1}^{4}}(7 \sigma)^{4} \varepsilon^{4}+\frac{\varepsilon_{j+1}^{2}}{\delta_{1}^{4}}(7 \sigma)^{4} \varepsilon^{2}\right) \\
& \leqq 32 E_{0} K_{0}\left(\frac{7 \sigma}{\sigma_{1}}\right)^{4}\left(\frac{\varepsilon}{\varepsilon_{j+1}}\right)^{2}
\end{aligned}
$$

This is a fundamental bound on the effective potential $\mathscr{V}^{(j)}$, it describes precisely its pseudo-scaling properties. We formulate the most important conclusions of the above considerations in the proposition

Proposition 3.4. The effective potentials $\mathscr{V}^{(j)}$ for $j \leqq k-n_{1}$ are given by the formulas (3.45) with functions $f^{(j)}$ defined and analytic on the polydiscs (3.31) with $\delta_{0}=\delta_{1}=\sigma_{1} \varepsilon_{j+1}, \varepsilon_{0}=\varepsilon_{j+1}, v=v_{j}$, and satisfying the bounds (3.36). Therefore the effective potentials $\mathscr{V}^{(j)}$ are analytic on domains $\Psi_{\text {const }}^{c}(\sigma, \varepsilon)$ with $7 \sigma \leqq \sigma_{1}, \varepsilon \leqq \varepsilon_{j+1}$, and they satisfy the bounds

$$
\left|\mathscr{V}^{(j)}\left(\psi+\psi^{\prime}, v_{j}\left(h+h^{\prime}\right)\right)\right|<32 E_{0} K_{0}\left(7 K_{1} L^{\frac{\alpha}{2}} \frac{1}{\alpha}\right)^{4} \sigma^{4}\left(\frac{\varepsilon}{\varepsilon_{j}}\right)^{2}
$$

on those domains.

We apply the above proposition to the case $\varepsilon=\varepsilon_{k}, \sigma=C_{0} L^{j} \eta$. The assumptions are satisfied if $L^{k-j} \geqq 7 C_{0} K_{1} \frac{1}{\alpha}$. We take the integer $n_{1}$ as the smallest integer satisfying the inequality $L^{n_{1}} \geqq 7 C_{0} K_{1} \frac{1}{\alpha}$, and $k-j \geqq n_{1}$, or $j \leqq k-n_{1}$. We obtain the bound

$$
\left|\mathscr{V}^{(j)}\left(\psi+\psi^{\prime}, v_{j}\left(h+h^{\prime}\right)\right)\right|<32 E_{0} K_{0}\left(7 C_{0} K_{1} L^{\frac{\alpha}{2}} \frac{1}{\alpha}\right)^{4}\left(L^{j} \eta\right)^{d+2-2 \gamma} .
$$

In this bound we want to have power of $L^{j} \eta$ larger than $d$, so we assume $\gamma<1$. We have assumed also $\gamma<\frac{d-2}{2}$ in (H.3), so we can write both conditions together as $\gamma<\min \left\{1, \frac{d-2}{2}\right\}$. In this case we could take $\gamma_{0}=\min \left\{1, \frac{d-2}{2}\right\}$. It turns out that this is a correct choice of $\gamma_{0}$ in general, for all remaining cases we will study in the future. Denote for simplicity

$$
K_{\alpha}=32 K_{0}\left(7 C_{0} K_{1} L^{\frac{\alpha}{2}} \frac{1}{\alpha}\right)^{4}, \quad 2(1-\gamma)=\alpha_{2} .
$$

By (3.24) we have

\section{Corollary 3.5 .}

$$
\left|\mathscr{V}^{(j)}\left(\psi_{k}^{(j)}\left(y ; \psi+\psi^{\prime}, v_{k}\left(h+h^{\prime}\right)\right), v_{j}\left(h+h^{\prime}(y)\right)\right)\right| \leqq E_{0} K_{\alpha}\left(L^{j} \eta\right)^{d+\alpha_{2}}
$$

for $\left(\psi+\psi^{\prime}, h+h^{\prime}\right) \in \Xi_{k}^{c}\left(1, \varepsilon_{k}\right)$.

This is a basic bound on the expressions $\mathscr{V}^{(j)}\left(\psi_{k}^{(j)}(y), v_{j} h\right)$ which will contribute to the uniform bound on $\mathscr{E}_{k}$. If we change $k$ into $k+1$, the above bound is multiplied by the factor $L^{-d-\alpha_{1}}$. This means precisely that the expressions are irrelevant quantities.

Consider now terms of the second sum in (3.23). Take a term corresponding to some $j, y, X$, and consider the function $\mathscr{E}^{(j)}\left(y, X ; \psi_{j}, v_{j} h\right)$. We expand it around the 
constant configuration $\psi_{j}(y)$ up to an appropriate order. We analyze this expansion in most of a remaining part of this subsection, so for simplicity of notation we drop the index $j$. It turns out that we have to expand up to the fourth order, thus we write

$$
\begin{aligned}
\mathscr{E}(y, X ; \psi, v h)-\mathscr{E}(y, X ; \psi(y), v h) \\
=\left\langle\left(\frac{\partial}{\partial \psi} \mathscr{E}\right)(y, X ; \psi(y), v h), \delta \psi\right\rangle+\frac{1}{2}\left\langle\left(\frac{\partial^{2}}{\partial \psi \partial \psi} \mathscr{E}\right)(y, X ; \psi(y), v h), \delta \psi, \delta \psi\right\rangle \\
+\frac{1}{6}\left\langle\left(\frac{\partial^{3}}{\partial \psi \partial \psi \partial \psi} \mathscr{E}\right)(y, X ; \psi(y), v h), \delta \psi, \delta \psi, \delta \psi\right\rangle \\
\quad+\frac{1}{6} \int_{0}^{1} d t(1-t)^{3}\left\langle\left(\frac{\partial^{4}}{\partial \psi \partial \psi \partial \psi \partial \psi} \mathscr{E}\right)\left(y, X ; \psi_{t}, v h\right), \delta \psi, \delta \psi, \delta \psi, \delta \psi\right\rangle \\
\delta \psi(x)=\psi(x)-\psi(y), \psi_{t}(x)=(1-t) \psi(y)+t \psi(x)=\psi(y)+t \delta \psi(x)
\end{aligned}
$$

We will study successively the terms of the expansion. Our basic goal is to decompose them further into sums of relevant, marginal and irrelevant expressions, and to construct their analytic extensions.

Let us start with the last term of the expansion. By Lemma 3.2 the function $\mathscr{E}$ is analytic on a space $\widetilde{\Psi}^{c}\left(\delta_{0}, \varepsilon_{0}\right)$ with $\delta_{0}, \varepsilon_{0}$ satisfying the conditions of the lemma, in particular with $\delta_{0}=\delta_{1}=\sigma_{1} \varepsilon_{j+1}, \varepsilon_{0}=\varepsilon_{j+1}$. We choose this space as a maximal analyticity domain, and all other spaces will be contained in it. We would like to extend the last term in a natural way as an analytic function of $\left(\psi+\psi^{\prime}, h+h^{\prime}\right) \in$ $\widetilde{\Psi}^{c}(\delta, \varepsilon)$ for properly chosen $\delta, \varepsilon$. The derivative of $\mathscr{E}$ in that term depends on $\left(\psi+\psi^{\prime}\right)_{t}=\psi_{t}+\psi_{t}^{\prime}$, so we have to determine to what space these configurations belong. We have

$$
\partial^{1} \psi_{t}=t \partial^{1} \psi, \text { hence }\left|\partial^{1} \psi_{t}\right| \leqq\left|\partial^{1} \psi\right|<\delta,
$$

and obviously the same bound holds for $\partial^{1} \psi_{t}^{\prime}$. Next

$$
\psi_{t}^{2}(x)-1=(1-t)\left(\psi^{2}(y)-1\right)+t\left(\psi^{2}(x)-1\right)-t(1-t)(\psi(x)-\psi(y))^{2} .
$$

hence $\left|\psi_{t}^{2}(x)-1\right|<\delta+\frac{1}{4}|\psi(x)-\psi(y)|^{2}$. It is immediate to estimate $\delta \psi(x)=$ $\psi(x)-\psi(y)$ by $\left|\Gamma_{y, x}\right| \delta$, where $\Gamma_{y, x}$ is any unit lattice path connecting the points $y, x$ and lying inside the domain $X$. We would like to have an estimate in terms of $d_{j}(X)=d(X)$, so we have to choose $\Gamma_{y, x}$ properly. Take a tree graph $\Gamma$ satisfying the conditions of the definition (2.27), and two cubes of the cover $\pi_{j}^{\prime}$ containing the points $y, x$ and contained in $X$, e.g. $\square^{\prime}, \square^{\prime \prime} \in \pi_{j}^{\prime}, \square^{\prime}, \square^{\prime \prime} \subset X, y \in \square^{\prime}, x \in \square^{\prime \prime}$. By the conditions of $(2.27)$ the graph $\Gamma$ intersects both cubes, take points $x^{\prime}, x^{\prime \prime}$ belonging to the corresponding intersections. There exists a subgraph of the graph $\Gamma$ which is a path containing the two points $x^{\prime}, x^{\prime \prime}$. Denote by $\Gamma^{\prime}$ the part of this path from $x^{\prime}$ to $x^{\prime \prime}$, hence $\Gamma^{\prime} \subset \Gamma$ and $\left|\Gamma^{\prime}\right| \leqq|\Gamma|$. We approximate this path by a unit lattice path contained in $X$ in the following way. Start with the point $x^{\prime}, x^{\prime} \in \square^{\prime}$ and $\square$ is an open LM-cube, so there exists a unit cube $\Delta\left(y_{0}\right)$ such that $x^{\prime} \in \Delta\left(y_{0}\right)$, and the interior of $\Delta\left(y_{0}\right)$ is contained in $\square$. Denote by $\Gamma_{1}$ the part of $\Gamma^{\prime}$ starting at $x^{\prime}$ and with $\left|\Gamma_{1}\right|=1$. Obviously $\Gamma_{1} \subset \widetilde{\Delta}\left(y_{0}\right)$, where $\widetilde{\Delta}\left(y_{0}\right)$ is a union of $3^{d}$ unit cubes touching $\Delta\left(y_{0}\right)$. Denote by $x_{1}$ a final point of $\Gamma_{1}$, and let $y_{1}$ be a unit lattice point such that $x_{1} \in \Delta\left(y_{1}\right) \subset \tilde{\Delta}\left(y_{0}\right)$. The point $x_{1}$ lies inside $X$, hence the interior of $\Delta\left(y_{1}\right)$ is contained in $X$, and at least one of the unit lattice shortest paths connecting $y_{0}$ 
with $y_{1}$ is contained in $X$. Denote such a path by $\Gamma_{y_{0}, y_{1}}$, hence $\left|\Gamma_{y_{0}, y_{1}}\right| \leqq d$. Take now the point $x_{1}$ and denote by $\Gamma_{2}$ the part of $\Gamma^{\prime}$ starting at $x_{1}$ and with $\left|\Gamma_{2}\right|=1$. Then $\Gamma_{2} \subset \tilde{\Delta}\left(y_{1}\right)$, and we denote by $x_{2}$ a final point of $\Gamma_{2}$, and by $y_{2}$ a unit lattice point such that $x_{2} \in \Delta\left(y_{2}\right) \subset \tilde{\Delta}\left(y_{1}\right)$. The point $x_{2}$ and the interior of the cube $\Delta\left(y_{2}\right)$ are inside $X$, hence there exists a shortest path connecting the points $y_{1}, y_{2}$ and contained inside $X$. Denote such a path by $\Gamma_{y_{1}, y_{2}}$. We continue this construction until we reach a point $x_{p}$ in $\Gamma^{\prime}$ such that a length of the remaining part of the path $\Gamma^{\prime}$ starting at the point $x_{p}$ is $\leqq 1$. We denote this part by $\Gamma_{p+1}$. Of course the point $x^{\prime \prime}$ is its final point. It belongs to $\square$ ", hence there exists a unit lattice point $y_{p+1} \in \square$ " such that $x^{\prime \prime} \in \Delta\left(y_{p+1}\right)$, and a shortest path $\Gamma_{y_{p}, y_{p+1}}$ contained in $X$. Thus we have constructed a unit lattice path, a union of the paths $\Gamma_{y_{i}, y_{i+1}}, i=0,1, \ldots, p$, connecting the points $y_{0}, y_{p+1}$ and contained in $X$. It is an approximation of the path $\Gamma^{\prime}$, and we have the inequality

$$
\begin{aligned}
\left|\bigcup_{i=0}^{p} \Gamma_{y_{i}, y_{i+1}}\right| & =\sum_{i=0}^{p}\left|\Gamma_{y_{i}, y_{i+1}}\right| \leqq d(p+1)=d \sum_{i=1}^{p}\left|\Gamma_{i}\right|+d \\
& <d\left|\Gamma^{\prime}\right|+d
\end{aligned}
$$

Let us take a shortest path $\Gamma_{y, y_{0}}$ connecting $y, y_{0}$, and a shortest path $\Gamma_{y_{p+1}, x}$ connecting $y_{p+1}, x$. Of course $\Gamma_{y, y_{0}} \subset \square^{\prime}, \Gamma_{y_{p+1}, x} \subset \square^{\prime \prime}$, and

$$
\left|\Gamma_{y, y_{0}}\right| \leqq d L M-d, \quad\left|\Gamma_{y_{p+1}, x}\right| \leqq d L M-d .
$$

We combine these two paths with the previously constructed path and we get the following path from $y$ to $x$

$$
\Gamma_{y, x}=\Gamma_{y, y_{0}} \cup \bigcup_{i=0}^{p} \Gamma_{y_{n}, y_{i+1}} \cup \Gamma_{y_{p+1}, x} .
$$

It is a unit lattice path contained in $X$, and we have

$$
\left|\Gamma_{y, x}\right| \leqq 2(d L M-d)+d\left|\Gamma^{\prime}\right|+d<2 d L M+d(|\Gamma|-1) .
$$

Taking $\Gamma$ such that $|\Gamma|-1<M d(X)$, we obtain the bound

$$
\left|\Gamma_{y, x}\right| \leqq 2 d L M+d M d(X) \text {. }
$$

We use this path to estimate $\delta \psi(x)$, and we get

$$
\begin{aligned}
|\delta \psi(x)| & \leqq\left|\Gamma_{y, x}\right|\left|\partial^{1} \psi\right|_{\Gamma_{y, x}} \leqq(2 d L M+d M d(X))\left|\partial^{1} \psi\right|_{X} \\
& <(2 d L M+d M d(X)) \delta \text { for } x \in X .
\end{aligned}
$$

The identity (3.53) and the bound (3.55) imply

$$
\left|\psi_{t}^{2}(x)-1\right|<\delta+\frac{1}{4}(2 d L M+d M d(X))^{2} \delta^{2} \quad \text { for } x \in X .
$$

We require that $\psi_{t}$ belong to the space $\widetilde{\Psi}\left(\frac{1}{2} \delta_{0}\right)$ on $X$. This is implied, for example, by the conditions

$$
2 d L M \delta \leqq \frac{1}{2} \delta_{0}, \quad d M d(X) \delta \leqq \frac{1}{2} \delta_{0}
$$

We will discuss them in detail later on. Consider now the last condition defining the space $\widetilde{\Psi}(\delta)$. The inequality $1-\psi_{0} \cdot h<\frac{\delta^{2}}{v}$ is really restrictive for $\frac{\delta^{2}}{v}$ small, because if 
$\frac{\delta^{2}}{v} \geqq 1$ then $1-\psi_{0} \cdot h \leqq 2<\frac{(2 \delta)^{2}}{v}$ holds for all non-zero vectors $\psi$. If $\frac{\delta^{2}}{v}<1$, then the inequality $1-\psi_{0} \cdot h<\frac{\delta^{2}}{v}$ defines a convex spherical cone in the space $\mathbf{R}^{N}$, with an axis determined by the vector $h$. If $\psi(y), \psi(x)$ satisfy the inequality, then they belong to the cone and their convex combinations $(1-t) \psi(y)+t \psi(x)=\psi_{t}(x)$ belong to the cone also for all $t \in[0,1]$. Thus generally the configuration $\psi_{t}$ satisfies the condition $v\left(1-\left(\psi_{t}\right)_{0} \cdot h\right)<(2 \delta)^{2}$ on $X$, assuming that (3.57) holds, because then $\psi_{t} \neq 0$ on $X$. We have proved the following statement: if $(\psi, h) \in \widetilde{\Psi}(\delta)$ and the conditions (3.57) hold, then $\left(\psi_{t}, h\right)$ on $X$, satisfies the conditions defining the space $\widetilde{\Psi}\left(\frac{1}{2} \delta_{0}\right)$. We would like to extend this statement to complex configurations and spaces. We have (3.52) for $\partial^{1} \psi_{t}^{\prime}$ and obviously also $\left|\psi_{t}^{\prime}\right|<\varepsilon+\delta$. Consider now $\left(\psi_{t}+\psi_{t}^{\prime}\right)^{2}-\psi_{t}^{2}$. The identity (3.53) holds for complex configurations also, in particular for $\psi_{t}+\psi_{t}^{\prime}=\left(\psi+\psi^{\prime}\right)_{t}$. Subtracting corresponding identities we get

$$
\begin{aligned}
\left(\psi_{t}(x)\right. & \left.+\psi_{t}^{\prime}(x)\right)^{2}-\psi_{t}^{2}(x) \\
= & (1-t)\left(\left(\psi(y)+\psi^{\prime}(y)\right)^{2}-\psi^{2}(y)\right)+t\left(\left(\psi(x)+\psi^{\prime}(x)\right)^{2}-\psi^{2}(x)\right) \\
& -t(1-t)\left(\psi^{\prime}(x)-\psi^{\prime}(y)\right)\left(2(\psi(x)-\psi(y))+\left(\psi^{\prime}(x)-\psi^{\prime}(y)\right)\right), \text { hence } \\
& \left|\left(\psi_{t}(x)+\psi_{t}^{\prime}(x)\right)^{2}-\psi_{t}^{2}(x)\right|<\delta+\frac{3}{4}(2 d L M+d M d(X))^{2} \delta^{2}<\frac{1}{2} \delta_{0}
\end{aligned}
$$

if the conditions (3.57) hold. The configuration $h^{\prime}$ satisfies

$$
\left|h^{\prime}\right|<\frac{\delta^{2}}{v \varepsilon} \leqq \frac{1}{4 d L M} \delta_{0} \frac{\delta}{v \varepsilon}<\frac{1}{4} \frac{\delta_{0}^{2}}{v \varepsilon_{0}} \text { if } \frac{\delta}{\varepsilon} \leqq \frac{\delta_{0}}{\varepsilon_{0}} .
$$

Further

$$
\begin{gathered}
\psi_{t}(x) \cdot h^{\prime}(x)=\psi(x) \cdot h^{\prime}(x)-(1-t) \delta \psi(x) \cdot h^{\prime}(x), \text { hence } \\
\left|\psi_{t} \cdot h^{\prime}\right|<|\psi(x)| \frac{\delta^{2}}{v}+(2 d L M+d M d(X)) \delta \frac{\delta^{2}}{v \varepsilon}<2 \frac{\delta^{2}}{v}+2 \delta_{0} \frac{\delta}{v} \\
<\frac{1}{d L M} \frac{\delta_{0}^{2}}{v}<\frac{1}{8} \frac{\delta_{0}^{2}}{v}, \text { and } \\
\left|\left(\psi_{t}\right)_{0} \cdot h^{\prime}\right|<\frac{1}{8\left|\psi_{t}\right|} \frac{\delta_{0}^{2}}{v}<\frac{1}{4} \frac{\delta_{0}^{2}}{v} .
\end{gathered}
$$

The conditions defining the space $\tilde{\Psi}^{c}\left(\frac{1}{2} \delta_{0}, \varepsilon_{0}\right)$ are therefore satisfied on $X$, if (3.57) hold and $\varepsilon \leqq \varepsilon_{0}$. The configuration $\left(\psi_{t}+\psi_{t}^{\prime}, h+h^{\prime}\right)$ may be extended onto the whole lattice by the formula

$$
\left(\psi_{t}+\psi_{t}^{\prime}, h+h^{\prime}\right)_{X}=\chi_{X}\left(\psi_{t}+\psi_{t}^{\prime}, h+h^{\prime}\right)+\chi_{X^{\prime}}\left(\psi(y)+\psi^{\prime}(y), h+h^{\prime}(y)\right),
$$

and the extended configuration satisfies all the conditions defining the space $\widetilde{\Psi}^{c}\left(\frac{1}{2} \delta_{0}, \varepsilon_{0}\right)$ on the whole lattice, hence it belongs to this space. The above considerations are summarized in the following lemma

Lemma 3.6. If $\left(\psi+\psi^{\prime}, h+h^{\prime}\right) \in \widetilde{\Psi}^{c}(\delta, \varepsilon), \varepsilon \leqq \varepsilon_{0}, \frac{\delta}{\varepsilon} \leqq \frac{\delta_{0}}{\varepsilon_{0}}$ and the conditions (3.57) are satisfied, then $\left(\psi_{t}+\psi_{t}^{\prime}, h+h^{\prime}\right)_{X} \in \tilde{\Psi}^{c}\left(\frac{1}{2} \delta_{0}, \varepsilon_{0}\right)$ for $t \in[0,1]$.

The function (3.58) is a linear, therefore analytic function of $\left(\psi+\psi^{\prime}, h+h^{\prime}\right)$, and the lemma implies that the last term in (3.51) is an analytic function on $\tilde{\Psi}^{c}(\delta, \varepsilon)$, or rather that its natural extension is such a function. Let us determine a maximal 
analyticity domain. We take $\varepsilon=\varepsilon_{0}=\varepsilon_{j+1}$, and we use the conditions (3.57) in the following way: the first condition determines a maximal $\delta$ satisfying it, i.e. $\delta_{2}=(4 d L M)^{-1} \sigma_{1} \varepsilon_{j+1}=\sigma_{2} \varepsilon_{j+1}$, where $\sigma_{2}=\left(4 d K_{1} L M\right)^{-1} \alpha$, and the second condition is considered as a restriction on $X$, i.e. we assume that $X$ satisfies

$$
d(X) \leqq \frac{1}{2 d M} \frac{\delta_{0}}{\delta} .
$$

Thus the last term in (3.51) is an analytic function on the space $\tilde{\Psi}^{c}\left(\delta_{2}, \varepsilon_{0}\right)$, or on any space $\widetilde{\Psi}^{c}(\delta, \varepsilon)$ such that $\delta \leqq \delta_{2}, \varepsilon \leqq \varepsilon_{0}, \frac{\delta}{\varepsilon} \leqq \delta_{2}$.

Let us estimate this term. For the analytic extension of the derivative in this term we have

$$
\begin{aligned}
& \left\langle\left(\frac{\partial^{4}}{\partial \psi \partial \psi \partial \psi \partial \psi} \mathscr{E}\right)\left(y, X ;\left(\psi+\psi^{\prime}\right)_{t}, v\left(h+h^{\prime}\right)\right), \delta\left(\psi+\psi^{\prime}\right), \delta\left(\psi+\psi^{\prime}\right),\right. \\
& \left.\quad \delta\left(\psi+\psi^{\prime}\right), \delta\left(\psi+\psi^{\prime}\right)\right\rangle \\
& \quad=\left.\frac{d^{4}}{d \tau^{4}} \mathscr{E}\left(y, X ;\left(\psi+\psi^{\prime}\right)_{t}+\tau \delta\left(\psi+\psi^{\prime}\right), v\left(h+h^{\prime}\right)\right)\right|_{\tau=0} \\
& \quad=\frac{24}{2 \pi i} \int_{|\tau|=r} d \tau \frac{1}{\tau^{5}} \mathscr{E}\left(y, X ;\left(\psi+\psi^{\prime}\right)_{t}+\tau \delta\left(\psi+\psi^{\prime}\right), v\left(h+h^{\prime}\right)\right),
\end{aligned}
$$

where $r$ is a sufficiently small positive number. In order to get a best bound we have to take a maximal possible $r$. We find it from the requirement that

$$
\left(\left(\psi+\psi^{\prime}\right)_{t}+\tau \delta\left(\psi+\psi^{\prime}\right), h+h^{\prime}\right)_{X} \in \tilde{\Psi}^{c}\left(\delta_{0}, \varepsilon_{0}\right) \text { for all } \tau:|\tau| \leqq r,
$$

because then we use the analyticity of the function $\mathscr{E}$ on this space to conclude that the expression (3.60) is an analytic function of $\left(\psi+\psi^{\prime}, h+h^{\prime}\right)$ on the space $\widetilde{\Psi}^{c}\left(\delta_{2}, \varepsilon_{0}\right)$, and an analytic function of $\tau$ on the disc $\left\{\tau:|\tau|<r_{0}\right\}$, where $r_{0}$ is a supremum over all $r$ for which the above condition is satisfied. From Lemma 3.6 and the definition of the space $\widetilde{\Psi}^{c}\left(\delta_{2}, \varepsilon_{0}\right)$ it follows easily that the condition is satisfied if the following three bounds hold on $X$

$$
\begin{gathered}
\left|\tau \delta\left(\psi+\psi^{\prime}\right)\right| \leqq \frac{1}{2} \delta_{0}, \quad\left|\tau\left(\partial^{1} \psi+\partial^{1} \psi^{\prime}\right)\right| \leqq \frac{1}{2} \delta_{0}, \\
\left|2\left(\psi+\psi^{\prime}\right)_{t} \cdot \tau \delta\left(\psi+\psi^{\prime}\right)+\left(\tau \delta\left(\psi+\psi^{\prime}\right)\right)^{2}\right| \leqq \frac{1}{2} \delta_{0} .
\end{gathered}
$$

The expression on the left-hand side of the last inequality above can be bounded by

$$
\begin{aligned}
{\left[2\left(\left|\psi_{t}\right|+\left|\psi_{t}^{\prime}\right|\right)+\frac{1}{2} \delta_{0}\right]\left|\tau \delta\left(\psi+\psi^{\prime}\right)\right| } & \leqq\left(2 \sqrt{1+\frac{1}{2} \delta_{0}}+2 \varepsilon_{0}+\frac{5}{2} \delta_{0}\right)\left|\tau \delta\left(\psi+\psi^{\prime}\right)\right| \\
& \leqq 3\left|\tau \delta\left(\psi+\psi^{\prime}\right)\right|,
\end{aligned}
$$

for $\varepsilon_{0}, \delta_{0}$ sufficiently small, hence the three bounds hold if $\left|\tau \delta\left(\psi+\psi^{\prime}\right)\right| \leqq \frac{1}{6} \delta_{0}$. By (3.55) we have

$$
\left|\tau \delta\left(\psi+\psi^{\prime}\right)\right|<r(2 d L M+d M d(X)) 2 \delta \text { on } X,
$$


hence the last condition is satisfied if $r(2 d L M+d M d(X)) 2 \delta \leqq \frac{1}{6} \delta_{0}$, and the maximal value $r_{0}$ is given by

$$
r_{0}=\frac{1}{12(2 d L M+d M d(X))} \frac{\delta_{0}}{\delta}=\frac{1}{24 d L M\left(1+\frac{1}{2 L} d(X)\right)} \frac{\delta_{0}}{\delta} .
$$

From the inductive hypothesis (H.5) and the representation (3.60) we can bound the last term in (3.51) by

$$
\begin{aligned}
\frac{1}{r_{0}^{4}} E_{0} \exp (-\kappa d(X)) & \leqq(24 d L M)^{4} \exp \left(\frac{4}{2 L} d(X)\right)\left(\frac{\delta}{\delta_{0}}\right)^{4} E_{0} \exp (-\kappa d(X)) \\
& \leqq(24 d L M)^{4}\left(\frac{\delta}{\delta_{0}}\right)^{4} E_{0} \exp (-(\kappa-1) d(X)) .
\end{aligned}
$$

This bound holds on the space $\widetilde{\Psi}^{c}(\delta, \varepsilon)$ with $\delta, \varepsilon$ satisfying conditions $\delta \leqq \delta_{2}, \varepsilon \leqq \varepsilon_{0}$ and $\frac{\delta}{\varepsilon} \leqq \frac{\delta_{0}}{\varepsilon_{0}}$.

We consider now the above conditions and bounds for the spaces $\Psi^{c}(\sigma, \varepsilon)$. In particular by Lemma 3.2 values of the function $\psi_{k}^{(j)}$ are in the space $\Psi_{j}^{c}\left(C_{0} L^{j} \eta, \varepsilon_{k}\right)$, so we analyze the conditions and bounds for this space. We have

$$
\Psi_{j}^{c}\left(C_{0} L^{j} \eta, \varepsilon_{k}\right) \subset \Psi_{j}^{c}\left(C_{0} L^{j} \eta \varepsilon_{k}, \varepsilon_{k}\right),
$$

hence now $\delta=C_{0} L^{j} \eta \varepsilon_{k} \varepsilon=\varepsilon_{k}$. The first condition in (3.57) is equivalent to $\delta \leqq \delta_{2}=\sigma_{2} \varepsilon_{j+1}=\sigma_{2} L^{-\alpha} \varepsilon_{j}$, or

$$
L^{j} \eta \frac{\varepsilon_{k}}{\varepsilon_{j}} \leqq C_{0}^{-1} \sigma_{2} L^{-\alpha}=\left(4 d C_{0} K_{1} L^{1+\alpha} M\right)^{-1} \alpha .
$$

This can be written as a condition on $k-j$ of the form

$$
L^{(1+\alpha)(k-j)} \geqq 4 d C_{0} K_{1} L^{1+\alpha} M \frac{1}{\alpha} .
$$

We define $n_{1}$ as a smallest integer satisfying this inequality. It satisfies also the inequality in Proposition 3.4. Consider the second condition in (3.57), or the condition (3.59). It can be written as

$$
d(X)=d_{j}(X) \leqq \frac{\sigma_{1} \varepsilon_{j+1}}{2 d M C_{0} L^{j} \eta \varepsilon_{k}}=\frac{\alpha}{2 d C_{0} K_{1} L^{\alpha} M} L^{(1+\alpha)(k-j)} .
$$

From the definition of $n_{1}$ it follows that it is implied by the simpler condition

$$
d_{j}(X) \leqq 2 L L^{(1+x)\left(k-j-n_{1}\right)} .
$$

This gives a transparent condition on the tree-size of the admissible localization domains.

Making the corresponding substitutions in the bound (3.61) we obtain the expression

$$
\left(24 d C_{0} K_{1} L^{1+\alpha} M \frac{1}{\alpha}\right)^{4}\left(L^{j} \eta\right)^{4(1+\alpha)} E_{0} \exp \left(-(\kappa-1) d_{j}(X)\right) .
$$

Let us recall that we have denoted $\alpha=\frac{d-2}{2}-\gamma$, so $1+\alpha=\frac{d}{2}-\gamma$ and $4(1+\alpha)=2 d-4 \gamma$. We require that $4(1+\alpha)>d$, hence $d-4 \gamma>0$. Notice that we 
have assumed $\gamma<\gamma_{0}=\min \left\{1, \frac{d-2}{2}\right\}, \quad$ so $\quad d-4 \gamma=2\left(\frac{d-2}{2}-\gamma\right)+2(1-\gamma) \geqq$ $2 \alpha+\alpha_{2}>0$. Denote

$$
K_{\gamma}=\left(24 d C_{0} K_{1} L^{1+x} M \frac{1}{\alpha}\right)^{4} .
$$

We summarize the results on the last term in (3.51) in the following lemma.

Lemma 3.7. The last term in the expansion (3.51) can be extended to an anlaytic function defined on any space $\widetilde{\Psi}^{c}(\delta, \varepsilon)$ with $\delta \leqq \delta_{2}, \varepsilon \leqq \varepsilon_{0}, \frac{\delta}{\varepsilon} \leqq \frac{\delta_{2}}{\varepsilon_{0}}$, and it can be bounded on this space by the right-hand side of (3.61). If $k-j \geqq n_{1}$, where $n_{1}$ is the smallest integer satisfying the inequality $(3.62)$, then the space $\tilde{\Psi}^{c}\left(\delta_{2}, \varepsilon_{0}\right)$ contains $\Psi_{j}^{c}\left(C_{0} L^{j} \eta, \varepsilon_{k}\right)$, and the last term can be bounded on it by

$$
K_{x}\left(L^{j} \eta\right)^{d+\gamma_{2}} E_{0} \exp \left(-(\kappa-1) d_{J}(X)\right) .
$$

Notice that the above bound implies again that the last term is an irrelevant quantity.

Consider now the terms of the second sum in (3.23) for which the condition (3.63) is not satisfied, i.e. we have

$$
d_{j}(X)>\frac{\alpha}{2 d C_{0} K_{1} L^{\alpha} M} L^{(1+\alpha)(k-j)} .
$$

The corresponding functions $\mathscr{E}^{(j)}\left(y, X, \psi_{j}, v_{j} h\right)$ can be extended to analytic functions on the space $\widetilde{\Psi}^{c}\left(\delta_{1}, \varepsilon_{0}\right)$, and we have the bound

$$
\begin{aligned}
&\left|\mathscr{E}^{(j)}\left(y, X ; \psi_{j}+\psi_{j}^{\prime}, v_{j}\left(h+h^{\prime}\right)\right)-\mathscr{E}^{(j)}\left(y, X ; \psi_{j}(y) \psi_{j}^{\prime}(y), v_{j}\left(h+h^{\prime}(y)\right)\right)\right| \\
& \leqq 2 E_{0} \exp \left(-\kappa d_{j}(X)\right)<2 E_{0} 4 !\left(2 d C_{0} K_{1} L^{\alpha} M \frac{1}{\alpha}\right)^{4}\left(L^{j} \eta\right)^{4(1+\gamma)} . \\
& \cdot \exp \left(-(\kappa-1) d_{j}(X)\right)=\frac{1}{3 \cdot 12^{2} L^{4}} K_{x}\left(L^{j} \eta\right)^{d+\alpha_{2}} E_{0} \exp \left(-(\kappa-1) d_{j}(X)\right) .
\end{aligned}
$$

Thus these terms have the same bound as the last term in the expansion (3.51), and they are irrelevant quantities also.

The remaining three terms in the expansion (3.51) cannot be shown to be irrelevant by such straightforward bounds as above. They demand a much more detailed analysis in order to insure the irrelevance. We start this analysis with bounds on derivatives occurring in the three terms. We need such bounds in slightly more general situations. We have

$$
\begin{aligned}
& \left\langle\left(\frac{\partial^{n}}{(\partial \psi)^{n}} \mathscr{E}\right)\left(y, X ; \psi+\psi^{\prime}, v\left(h+h^{\prime}\right)\right), \delta \psi_{1}, \ldots, \delta \psi_{n}\right\rangle \\
& =\left.\frac{\partial^{n}}{\partial \tau_{1} \cdots \partial \tau_{n}} \mathscr{E}\left(y, X ; \psi+\psi^{\prime}+\sum_{i=1}^{n} \tau_{l} \delta \psi_{i}, \nu^{\prime}\left(h+h^{\prime}\right)\right)\right|_{\tau_{1}=\tau_{n}=0} \\
& =\frac{1}{2 \pi i} \int_{\left|\tau_{1}\right|=1} \frac{d \tau_{1}}{\tau_{1}^{2}} \cdots \frac{1}{2 \pi i} \int_{\left|\tau_{n}\right|=r_{n}} \frac{d \tau_{n}}{\tau_{n}^{2}} \mathscr{E}\left(y, X ; \psi+\psi^{\prime}+\sum_{i=1}^{n} \tau_{i} \delta \psi_{i}, v\left(h+h^{\prime}\right)\right) .
\end{aligned}
$$

We take $\left(\psi+\psi^{\prime}, h+h^{\prime}\right) \in \widetilde{\Psi}^{c}\left(\frac{1}{2} \delta_{0}, \varepsilon_{0}\right), \delta \psi_{i}$ are arbitrary configurations with values in $\mathbf{C}^{N}$, and $r_{i}$ are sufficiently small. We find them from the condition that the configuration inside the last function above belongs to the space $\widetilde{\Psi}^{c}\left(\delta_{0}, \varepsilon_{0}\right)$. By the 
same analysis as in the proof of (3.61) we can show that this condition is satisfied if

$$
\left|\sum_{i=1}^{n} \tau_{i} \delta \psi_{i}\right| \leqq \frac{1}{6} \delta_{0} \text { on } X \text {, hence if } r_{i} \leqq \frac{\delta_{0}}{6 n\left|\delta \psi_{i}\right|_{X}} .
$$

From this we obtain the bound

$$
\begin{gathered}
\left|\left\langle\left(\frac{\partial^{n}}{(\partial \psi)^{n}} \mathscr{E}\right)\left(y, X ; \psi+\psi^{\prime}, v\left(h+h^{\prime}\right)\right), \delta \psi_{1}, \ldots, \delta \psi_{n}\right\rangle\right| \\
\leqq\left(\frac{6 n}{\delta_{0}}\right)^{n} E_{0} \exp (-\kappa d(X))\left|\delta \psi_{1}\right|_{x} \cdots\left|\delta \psi_{n}\right|_{x}
\end{gathered}
$$

holding on $\tilde{\Psi}^{c}\left(\frac{1}{2} \delta_{0}, \varepsilon_{0}\right)$ and for arbitrary $\delta \psi_{i}$.

We need to consider the derivatives for constant configurations only, i.e. we take $\left(\psi+\psi^{\prime}, h+h^{\prime}\right) \in \tilde{\Psi}_{\text {const }}^{c}\left(\frac{1}{2} \delta_{0}, \varepsilon_{0}\right)$. Let us study symmetry properties and their consequences. The inductive assumption (H.4) and the formula (3.67) imply

$$
\begin{gathered}
\left\langle\left(\frac{\partial^{n}}{(\partial \psi)^{n}} \mathscr{E}\right)\left(y, X ; R\left(\psi+\psi^{\prime}\right), v R\left(h+h^{\prime}\right)\right), R \delta \psi_{1}, \ldots, R \delta \psi_{n}\right\rangle \\
=\left\langle\left(\frac{\partial^{n}}{(\partial \psi)^{n}} \mathscr{E}\right)\left(y, X ; \psi+\psi^{\prime}, v\left(h+h^{\prime}\right)\right), \delta \psi_{1} \cdots \delta \psi_{n}\right\rangle
\end{gathered}
$$

for $R \in O(N)$. We take $\psi^{\prime}=0, h^{\prime}=0$, and we use the above invariance in the same way as for the effective potentials in the proof of (3.25), we take the same $R=R_{2} R_{1}$ and we obtain

$$
\begin{aligned}
& \left\langle\left(\frac{\partial^{n}}{(\partial \psi)^{n}} \mathscr{E}\right)(y, X ; \psi, v h), \delta \psi_{1}, \ldots, \delta \psi_{n}\right\rangle \\
& =\left\langle( \frac { \partial ^ { n } } { ( \partial \psi ) ^ { n } } \mathscr { E } ) \left( y, X ;|\psi| e_{1}, v\left(\psi_{0} \cdot h\right) e_{1}\right.\right. \\
& \left.\left.\quad+v \sqrt{|h|^{2}-\left(\psi_{0} \cdot h\right)^{2}} e_{2}\right), R \delta \psi_{1}, \ldots, R \delta \psi_{n}\right\rangle .
\end{aligned}
$$

The expression on the right-hand side obviously has the invariance property (3.69), in particular with respect to all the transformation $R^{\prime \prime} \in O(N)$ leaving invariant the vectors $e_{1}, e_{2}$. By the form of this expression it means that it is invariant with respect to the transformations $R^{\prime \prime}$ acting on the configurations $R \delta \psi_{1}$ only. Consider the functions

$$
\left(\frac{\partial^{n}}{\partial \psi\left(x_{1}\right) \ldots \partial \psi\left(x_{n}\right)} \mathscr{E}\right)\left(y, X ; u e_{1}, v v e_{1}+v w e_{2}\right) .
$$

They are defined and analytic on the domain (3.26) with $\frac{1}{2} \delta_{0}$ instead of $\delta_{0}$, but we are interested again in a neighborhood of the point $u=1, v=1, w=0$, so we consider it on the domain (3.29) with the same $\frac{1}{2} \delta_{0}$. The functions are analytic on this domains and satisfy the bound (3.68). By the preceding remark they have the symmetry property

$$
\begin{aligned}
& \left(\otimes^{n} R^{\prime \prime}\right)\left(\frac{\partial^{n}}{(\partial \psi)^{n}} \mathscr{E}\right)\left(y, X ; u e_{1}, v v e_{1}+v w e_{2}\right) \\
& =\left(\frac{\partial^{n}}{(\partial \psi)^{n}} \mathscr{E}\right)\left(y, X ; u e_{1}, v v e_{1}+v w e_{2}\right)
\end{aligned}
$$


for all $R^{\prime \prime} \in O(N)$ leaving invariant the vectors $e_{1}, e_{2}$. Of course they have also the more general property (3.69). Now we study consequences of this symmetry for the three cases $n=1,2,3$ which are interesting for us. These consequences become more complicated with increasing $n$, although they are quite elementary.

Take $n=1$. The first order derivative is a vector invariant with respect to the transformations $R^{\prime \prime}$, hence it is a linear combination of the vectors $e_{1}, e_{2}$. Applying (3.69) with $R$ equal to the reflection with respect to the $e_{2}$ component, we obtain that the $e_{1}$ component of the derivative is an even function of $w$, and the $e_{2}$ component is an odd function of $w$. Thus we can write

$$
\begin{aligned}
\left(\frac{\partial}{\partial \psi(x)} \mathscr{E}\right)\left(y, X ; u e_{1}, v v e_{1}+v w e_{2}\right)= & v_{1}\left(x, y, X ; u, v v, v^{2} w^{2}\right) u e_{1} . \\
& +v_{2}\left(x, y, X ; u, v v, v^{2} w^{2}\right) v w e_{2} .
\end{aligned}
$$

and $v_{1}, v_{2}$ are analytic functions on the domain (3.29) with $\frac{1}{2} \delta_{0}$ instead of $\delta_{0}$. The function $v_{1} u$ satisfies the bound (3.68) with $n=1$ and $e_{1} \cdot \delta \psi_{1}$ instead of $\delta \psi_{1}$. For the function $v_{2}$ we have the representation

$$
v_{2}\left(x, y, X ; u, v v, v^{2} w^{2}\right)=\frac{1}{v w}\left(\frac{\partial}{\partial \psi(x)} \mathscr{E}\right)\left(y, X ; u e_{1}, v v e_{1}+v w e_{2}\right) \cdot e_{2},
$$

so it satisfies the bound (3.68) with $n=1$, and with an additional factor $\sqrt{2} \frac{\varepsilon_{0}}{\left(\frac{1}{2} \delta_{0}\right)^{2}}$. The function $\delta \psi_{1}$ is replaced by $e_{2} \cdot \delta \psi_{1}$. For further reference let us write the domain (3.29) with $\frac{1}{2} \delta_{0}$ instead of $\delta_{0}$ explicitly in the form

$$
|u-1|<\frac{1}{6} \delta_{0}, \quad|v-1|<\frac{1}{4} \frac{\delta_{0}^{2}}{v}, \quad\left|w^{2}\right|<\frac{1}{32}\left(\frac{\delta_{0}^{2}}{v \varepsilon_{0}}\right)^{2} .
$$

Guided by the analysis of the effective potentials, and by the fact that the function $v_{1}$ is multiplied by $e_{1} \cdot \delta \psi$, we expect that it is enough to expand $v_{1}$ up to the first orders in $u-1, v(v-1)$ and $v^{2} w^{2}$, to get irrelevant expressions. The function $v_{2}$ is multiplied by $v w e_{2} \cdot \delta \psi$, which corresponds to a marginal expression, so it is enough also to expand up to the first orders in all the variables. Thus we write

$$
\begin{aligned}
v_{\sigma}\left(x, y, X ; u, v v, v^{2} w^{2}\right)= & v_{\sigma}(x, y, X ; 1, v, 0)+v_{\sigma ; 1,0,0}\left(x, y, X ; u, v v, v^{2} w^{2}\right)(u-1) \\
& +v_{\sigma ; 0,1,0}\left(x, y, X ; u, v v, v^{2} w^{2}\right) v(v-1) \\
& +v_{\sigma ; 0,1,0}\left(x, y, X ; u, v v, v^{2} w^{2}\right) v^{2} w^{2} .
\end{aligned}
$$

The functions $v_{\sigma, \alpha, \beta, \gamma}$ are expressed in terms of the corresponding derivatives of the functions $v_{\sigma}$ by Taylor's formula in the integral, or by the corresponding difference quotients, which is actually more convenient for bounds. Using the last representation we obtain easily the bounds

$$
\begin{aligned}
& \left|\left\langle v_{\sigma, \alpha, \beta, \gamma}\left(y, X ; u, v v, v^{2} w^{2}\right), \delta \zeta\right\rangle\right| \\
& \quad \leqq 2 \cdot 6^{\sigma+\alpha} 4^{\beta} 32^{\gamma} \frac{\varepsilon_{0}^{\sigma-1+2 \gamma}}{\delta_{0}^{2 \sigma-1+\alpha+2 \beta+4 \gamma}} E_{0} \exp (-\kappa d(X))|\delta \zeta|_{X}
\end{aligned}
$$

on the domain (3.75) and for $\alpha, \beta, \gamma$ such that $\alpha+\beta+\gamma \leqq 1$. The function $\delta \zeta$ is an arbitrary complex valued function defined on $X$. 
Take $n=2$. The second order derivative in components of $\psi$ is a matrix commuting with all the matrices $R^{\prime \prime}$, by the symmetry (3.51). This implies that it is a block matrix with two blocks. The first block is a $2 \times 2$-matrix in components with indices 1,2 , the second block is a $(N-2) \times(N-2)$-matrix in components with indices $3, \ldots, N$, commuting with all orthogonal matrices in those components, so it is proportional to the identity matrix. Denote the first block by $\left[\begin{array}{ll}a & b \\ c & d\end{array}\right]$, and the second block by $e I$. The second order derivative has also the general covariance property (3.69). Applying it to the reflection in the component of $e_{2}$, we obtain that $a, d, e$ are even functions of $w$, and $b, c$ are odd functions of $w$, so $b(w=0)=c(w=0)=0$. Take now the second order derivative at $w=0$. It has the invariance property (3.72) with $R^{\prime \prime}$ replaced by $R^{\prime}$, where $R^{\prime} \in O(N)$ is an arbitrary transformation leaving the vector $e_{1}$ invariant. This invariance implies that the corresponding matrix has also a block structure with two blocks, the first is just the element $a(w=0)$, the second is a $(N-1) \times(N-1)$-matrix proportional to the identity matrix. The coefficient multiplying the matrix must be equal to $d(w=0)$ and $e(w=0)$, hence $d(w=0)=e(w=0)$. Thus the matrix representing the second order derivative is a sum of two matrices, one is the matrix $e I$, where $I$ is now the identity matrix in all components, and another is a matrix which in the first two components is equal to $\left[\begin{array}{cc}a-e & b \\ c & d-e\end{array}\right]$, and all the remaining elements are equal to 0 . Notice that $d-e$ is an even function of $w$, and it is equal to 0 at $w=0$, so we can write $d-e=d_{1} v^{2} w^{2}$. The above considerations lead to the following representation of the second order derivative:

$$
\begin{aligned}
& \left(\frac{\partial^{2}}{\partial \psi\left(x_{1}\right) \partial \psi\left(x_{2}\right)} \mathscr{E}\right)\left(y, X ; u e_{1}, v v e_{1}+v w e_{2}\right) \\
& =v_{0}\left(x_{1}, x_{2}, y, X ; u, v v, v^{2} w^{2}\right) I+v_{1,1}\left(x_{1}, x_{2}, y, X ; u, v v, v^{2} w^{2}\right) u^{2} e_{1} \otimes e_{1} \\
& \quad+v_{1,2}\left(x_{1}, x_{2}, y, X ; u, v v, v^{2} w^{2}\right) u v w e_{1} \otimes e_{2} \\
& \quad+v_{2,1}\left(x_{1}, x_{2}, y, X ; u, v v, v^{2} w^{2}\right) v w u e_{2} \otimes e_{1} \\
& \quad+v_{2,2}\left(x_{1}, x_{2}, y, X ; u, v v, v^{2} w^{2}\right) v^{2} w^{2} e_{2} \otimes e_{2} .
\end{aligned}
$$

The functions $v_{0}, v_{\sigma, \rho}, \sigma, \rho=1,2$, can be written as the corresponding linear combinations of matrix elements of the second order derivative. For example we have

$$
\begin{aligned}
& v_{2,2}\left(x_{1}, x_{2}, y, X ; u, v v, v^{2} w^{2}\right) \\
& =\frac{1}{v^{2} w^{2}} \operatorname{tr}\left[\left(\frac{\partial^{2}}{\partial \psi\left(x_{1}\right) \partial \psi\left(x_{2}\right)} \mathscr{E}\right)\left(y, X ; u e_{1}, v v e_{1}+v w e_{2}\right)\left(e_{2} \otimes e_{2}-e_{N} \otimes e_{N}\right)\right] \\
& =\int_{0}^{1} d t(1-t) \operatorname{tr}\left[\left(\frac{\partial^{2}}{\partial(v w)^{2}} \frac{\partial^{2}}{\partial \psi\left(x_{1}\right) \partial \psi\left(x_{2}\right)} \mathscr{E}\right)\left(y, X ; u e_{1}, v v e_{1}+t v w e_{2}\right)\right. \\
& \left.\quad \times\left(e_{2} \otimes e_{2}-e_{N} \otimes e_{N}\right)\right],
\end{aligned}
$$


and similar, but simpler and obvious formulas for the remaining functions. From these formulas we obtain that the functions satisfy the bounds (3.68) with some additional factors, which we will write below. In the expansion (3.51) the second order derivative of $\mathscr{E}$ occurs with the second power of $\delta \psi$, so it is at least a marginal quantity. We will see that the last four terms in the representation (3.78) provide some additional scaling factors, so they are actually irrelevant quantities. We expand the function $v_{0}$ up to the first order in all variables writing the same formulas as (3.76) for $v_{\sigma}$, and we obtain the bounds

$$
\begin{aligned}
& \left|\left\langle v_{0 ; \alpha, \beta, \gamma}\left(y, X ; u, v v, v^{2} w^{2}\right), \delta \zeta_{1}, \delta \zeta_{2}\right\rangle\right| \\
& \quad \leqq 2 \cdot 12^{2} 6^{\alpha} 4^{\beta} 32^{\gamma} \frac{\varepsilon_{0}^{2 \gamma}}{\delta_{0}^{2+\alpha+2 \beta+4 \gamma}} E_{0} \exp (-\kappa d(X))\left|\delta \zeta_{1}\right|_{X}\left|\delta \zeta_{2}\right|_{X}
\end{aligned}
$$

on the domain (3.75), where $\alpha+\beta+\gamma \leqq 1$ and $\delta \zeta_{1}, \delta \zeta_{2}$ are arbitrary complex valued functions on $X$. Similarly for the functions $v_{\sigma, \rho}$ we obtain

$$
\begin{aligned}
& \left|\left\langle v_{\sigma, \rho}\left(y, X ; u, v v, v^{2} w^{2}\right) \delta \zeta_{1}, \delta \zeta_{2}\right\rangle\right| \\
& \quad \leqq 8 \cdot 6^{\sigma+\rho} \frac{\varepsilon_{0}^{\sigma+\rho-2}}{\delta_{0}^{2 \sigma+2 \rho-2}} E_{0} \exp (-\kappa d(X))\left|\delta \zeta_{1}\right|_{X}\left|\delta \zeta_{2}\right|_{X}
\end{aligned}
$$

on the domain (3.75), $\sigma, \rho=1,2$, and $\delta \zeta_{1}, \delta \zeta_{2}$ as above.

Applying the formulas (3.70), (3.73), (3.78) to the terms of the expansion (3.51) we get a sum of terms in which the configuration $\delta \psi$ is replaced by $|\psi(y)| e_{1} \cdot R \delta \psi$ or $v \sqrt{|h|^{2}-\left(\psi_{0}(y) \cdot h\right)^{2}} e_{2} \cdot \mathrm{R} \delta \psi$. By the definition of $R$ we have $R \psi(y)=|\psi(y)| e_{1}$, hence $R \delta \psi(x)=R \psi(x)-|\psi(y)| e_{1}$, and

$$
\begin{aligned}
|\psi(y)| e_{1} \cdot R \delta \psi(x) & =\psi(y) \cdot \delta \psi(x)=\psi(y) \cdot(\psi(x)-\psi(y)) \\
& =-\frac{1}{2}|\psi(x)-\psi(y)|^{2}+\frac{1}{2}|\psi(x)|^{2}-\frac{1}{2}|\psi(y)|^{2} \\
& =Z_{1}(x, y, \psi) .
\end{aligned}
$$

For the second expression we have

$$
\begin{aligned}
v \sqrt{|h|^{2}-\left(\psi_{0}(y) \cdot h\right)^{2}} e_{2} \cdot R \delta \psi(x)= & v\left|\left(R_{1} h\right)^{\prime}\right| e_{2} \cdot\left(R \psi(x)-\left(R \psi(x) \cdot e_{1}\right) e_{1}\right) \\
= & v R_{2}\left(R_{1} h\right)^{\prime} \cdot\left(R \psi(x)-\left(R \psi(x) \cdot e_{1}\right) e_{1}\right) \\
= & v\left(R_{1} h\right)^{\prime} \cdot\left(R_{1} \psi(x)-\left(R_{1} \psi(x) \cdot e_{1}\right) e_{1}\right) \\
= & v\left(\left(R_{1} h \cdot e_{1}\right) e_{1}+\left(R_{1} h\right)^{\prime}\right) \cdot\left(R_{1} \psi(x)\right. \\
& \left.-\left(R_{1} \psi(x) \cdot e_{1}\right) e_{1}\right) \\
= & v R_{1} h \cdot\left(R_{1} \psi(x)-\left(R_{1} \psi(x) \cdot e_{1}\right) e_{1}\right) \\
= & v h \cdot\left(\psi(x)-\left(\psi(x) \cdot \psi_{0}(y)\right) \psi_{0}(y)\right) \\
= & v h \cdot\left(I-\psi_{0}(y) \otimes \psi_{0}(y)\right) \psi(x) .
\end{aligned}
$$

We have used here the definitions of $R_{1}, R_{2}$ and $R=R_{2} R_{1}$ given between the formulas (3.24) and (3.25). It is not clear from the expression above that it is 
marginal, so we transform it further

$$
\begin{aligned}
v h \cdot\left(I-\psi_{0}(\psi) \otimes \psi_{0}(y)\right) \psi(x) \\
=\left(v h \cdot \psi_{0}(x)-v h \cdot \psi_{0}(y)\right)+(|\psi(x)|-1) \\
\quad \cdot\left(v h \cdot \psi_{0}(x)-v h \cdot \psi_{0}(y)\right)+\frac{1}{2} v h \cdot \psi_{0}(y)|\psi(x)|\left|\psi_{0}(x)-\psi_{0}(y)\right|^{2} \\
=\left(v h \cdot \psi_{0}(x)-v h \cdot \psi_{0}(y)\right)+(|\psi(x)|-1)\left(v h \cdot \psi_{0}(x)-v h \cdot \psi_{0}(y)\right) \\
\quad+\frac{v h \cdot \psi_{0}(y)}{2|\psi(y)|}\left(|\psi(x)-\psi(y)|^{2}-(|\psi(x)|-|\psi(y)|)^{2}\right)=\left(v h \cdot \psi_{0}(x)\right. \\
\left.\quad-v h \cdot \psi_{0}(y)\right)+\frac{1}{2} v|\psi(x)-\psi(y)|^{2}+R_{1}(x, y, \psi, v h)=Z_{2}\left(x_{1}, y, \psi, v h\right) .
\end{aligned}
$$

The expression in the parenthesis on the right-hand side is marginal, the remaining expressions are irrelevant. We have separated the second term, although it is basically irrelevant because of the factor $v$, having in mind some simplifications in the future. The expressions $Z_{1}, Z_{2}$ are simple algebraic functions of $(\psi, h)$ and they can be extended to analytic functions of $\left(\psi+\psi^{\prime}, h+h^{\prime}\right)$ on large domains, certainly on $\Psi^{c}(\delta, \varepsilon)$ for $\varepsilon$ sufficiently small. We want to estimate their restrictions to $X$. For $Z_{1}$ it is simple, by (3.55) we obtain

$$
\begin{aligned}
\left|Z_{1}\left(x, y, \psi+\psi^{\prime}\right)\right| & <\frac{1}{2}(2 d L M+d M d(X))^{2}(2 \sigma \varepsilon)^{2}+2 \sigma^{2} \varepsilon \\
& =2 \sigma^{2} \varepsilon\left(1+(2 d L M)^{2}\left(1+\frac{1}{2 L} d(X)\right)^{2} \varepsilon\right) \\
& \leqq 4 \sigma^{2} \varepsilon\left(1+\frac{1}{2 L} d(X)\right)^{2},
\end{aligned}
$$

where we have assumed that $(2 d L M)^{2} \varepsilon \leqq 1$. The constant $\varepsilon$ is one of the $\varepsilon_{j}$ 's, so we assume simply that $(2 d L M)^{2} \alpha_{0} \leqq 1$. We will have to make strong assumptions on $\alpha_{0}$ in the future. The expression $Z_{2}$ can be written in terms of the functions $U, V$ defined in (3.37),

$$
\begin{aligned}
Z_{2}(x, y)= & (v V(x)-v V(y))+\frac{1}{2} v\left(\delta\left(\psi+\psi^{\prime}\right)\right)^{2}+R_{1}(x, y), \\
R_{1}(x, y)= & (U(x)-1)(v V(x)-v V(y))-\frac{1}{2} v(U(x)-U(y))^{2} \\
& +\frac{v(V(y)-1)+v(1-U(y))}{2 U(y)}\left(\left(\delta\left(\psi+\psi^{\prime}\right)\right)^{2}-(U(x)-U(y))^{2}\right) .
\end{aligned}
$$

From the inequalities (3.39), (3.41) and (3.55) we obtain immediately the bound

$$
\begin{aligned}
\left|R_{1}(x, y)\right|< & 88 \sigma^{4} \varepsilon^{3}+8 v \sigma^{4} \varepsilon^{2}+\left(22 \sigma^{2} \varepsilon^{2}+2 v \sigma^{2} \varepsilon\right) \\
& \times\left[16 \sigma^{4} \varepsilon^{2}+(2 d L M+d M d(X))^{2} 4 \sigma^{2} \varepsilon^{2}\right] \\
< & 10 \sigma^{4} \varepsilon^{2}\left(1+\frac{1}{2 L} d(X)\right)^{2},
\end{aligned}
$$


where we have assumed that $15(2 d L M)^{2} \varepsilon \leqq 1$. To estimate the first two terms on the right-hand side of (3.85) we use additionally the assumption $v \leqq \sigma^{2}$ and we get

$$
\begin{gathered}
|v V(x)-v V(y)|<44 \sigma^{2} \varepsilon^{2} \\
\left|\frac{1}{2} v\left(\delta\left(\psi+\psi^{\prime}\right)\right)^{2}\right|<2 \sigma^{4} \varepsilon^{2}(2 d L M)^{2}\left(1+\frac{1}{2 L} d(X)\right)^{2} .
\end{gathered}
$$

Combining the estimates (3.86), (3.87) we obtain for example

$$
\left|Z_{2}\left(x, y, \psi+\psi^{\prime}, v\left(h+h^{\prime}\right)\right)\right|<44 \sigma^{2} \varepsilon^{2}+3 \sigma^{4} \varepsilon^{2}(2 d L M)^{2}\left(1+\frac{1}{2 L} d(X)\right)^{2} .
$$

The bounds (3.84), (3.88) hold for $x, y \in X$, and they are a basis of an estimate of the first two terms in the expansion (3.51).

Let us write now the sum of these two terms using the above representations and expansions. We have obtained the following equality:

$$
\begin{aligned}
\left\langle\left(\frac{\partial}{\partial \psi} \mathscr{E}\right)\right. & (y, X ; \psi(y), v h), \delta \psi\rangle+\frac{1}{2}\left\langle\left(\frac{\partial^{2}}{\partial \psi \partial \psi} \mathscr{E}\right)(y, X ; \psi(y), v h), \delta \psi, \delta \psi\right\rangle \\
= & \left\langle v_{1}(y, X ; 1, v, 0), Z_{1}(y, \psi)\right\rangle+\left\langle v_{2}(y, X ; 1, v, 0), v h \cdot \psi_{0}-v h \cdot \psi_{0}(y)\right\rangle \\
& +\frac{1}{2} v\left\langle v_{2}(y, X ; 1, v, 0),|\psi-\psi(y)|^{2}\right\rangle+\left\langle v_{2}(y, X ; 1, v, 0), R_{1}(y, \psi, v h)\right\rangle \\
& +\sum_{\sigma=1,2} \sum_{\alpha+\beta+\gamma=1}\left\langle v_{\sigma: \alpha, \beta, \gamma}\left(y, X ;|\psi(y)|, v h \cdot \psi_{0}(y), v^{2}\left(1-\left(h \cdot \psi_{0}(y)\right)^{2}\right)\right)\right. \\
& \left.\cdot(|\psi(y)|-1)^{\alpha}\left(v\left(h \cdot \psi_{0}(y)-1\right)\right)^{\beta}\left(v^{2}\left(1-\left(h \cdot \psi_{0}(y)\right)^{2}\right)\right)^{\gamma}, Z_{\sigma}(y, \psi, v h)\right\rangle \\
& +\frac{1}{2}\left\langle v_{0}(y, X ; 1, v, 0), \delta \psi, \delta \psi\right\rangle \\
& +\frac{1}{2} \sum_{\alpha+\beta+\gamma=1}\left\langle v_{0, \alpha . \beta, \gamma}\left(y, X ;|\psi(y)|, v h \cdot \psi_{0}(y), v^{2}\left(1-\left(h \cdot \psi_{0}(y)\right)^{2}\right)\right)\right. \\
& \left.\cdot(|\psi(y)|-1)^{\alpha}\left(v\left(h \cdot \psi_{0}(y)-1\right)\right)^{\beta}\left(v^{2}\left(1-\left(h \cdot \psi_{0}(y)\right)^{2}\right)\right)^{\gamma}, \delta \psi, \delta \psi\right\rangle \\
& +\frac{1}{2} \sum_{\sigma, \rho=1,2}\left\langle v_{\sigma, \rho}\left(y, X ;|\psi(y)|, v h \cdot \psi_{0}(y), v^{2}\left(1-\left(h \cdot \psi_{0}(y)\right)^{2}\right)\right),\right. \\
& \left.\times Z_{\sigma}(y, \psi, v h), Z_{\rho}(y, \psi, v h)\right\rangle .
\end{aligned}
$$

We will analyze in detail the terms on the right-hand side of this equality. Let us mention that the third term in the expansion (3.51) does not demand such a detailed analysis, so we will treat it separately in a simpler way.

Let us start the analysis with estimates of the three sums in (3.89). All terms of those sums have obvious analytic extensions through the functions $U, V, W^{2}$ on the spaces $\Psi^{c}(\sigma, \varepsilon)$ with $\sigma, \varepsilon$ satisfying proper conditions. We have discussed this already in the case of the effective potentials, and only small adjustments are 
needed here. We require that the three functions map analytically the corresponding domain $\Psi_{\text {const }}^{c}(\sigma, \varepsilon)$ into the polydisc (3.75). From the inequalities (3.39), (3.41), (3.43) we conclude that the following bounds hold on the domain $\Psi_{c o n s t}^{c}(\sigma, \varepsilon)$ :

$$
|U-1|<\frac{1}{6}\left(10 \sigma^{2}\right) \varepsilon,|V-1|<\frac{1}{4} \frac{(10 \sigma)^{2} \varepsilon^{2}}{v},\left|W^{2}\right|<\frac{1}{32} \frac{(10 \sigma)^{2} \varepsilon^{2}}{v} .
$$

Thus the functions $U, V, W^{2}$ map the domain $\Psi_{\text {const }}^{c}(\sigma, \varepsilon)$ into the polydisc (3.75) if $\varepsilon \leqq \varepsilon_{0}, 10 \sigma \varepsilon_{0} \leqq \delta_{0}$. We have $\delta_{0}=\sigma_{1} \varepsilon_{0}$, hence the last condition can be written as $10 \sigma \leqq \sigma_{1}$, or $10 K_{1} \frac{1}{\alpha} \sigma \leqq 1$. In the future we will always assume that these conditions are satisfied for considered spaces.

Consider the first sum on the right-hand side of (3.89). Its bound is straightforward; we use the inequalities (3.77), (3.84), (3.88) and (3.90). We can simplify a little bit the bound (3.88) using the condition $\sigma \leqq \frac{\alpha}{10 K_{1}}$; we have

$$
\left|Z_{2}\left(x, y, \psi+\psi^{\prime}, v\left(h+h^{\prime}\right)\right)\right|<\sigma^{2} \varepsilon^{2}(2 d L M)^{2}\left(1+\frac{1}{2 L} d(X)\right)^{2} \text { on } X .
$$

These inequalities imply that the first sum on the right-hand side of (3.89) can be bounded by

$$
\begin{aligned}
& \sum_{\alpha+\beta+\gamma=1} 12 \frac{\varepsilon_{0}^{2 \gamma}}{\delta_{0}^{1+\alpha+2 \beta+4 \gamma}} E_{0} \\
& \times \exp (-\kappa d(X))(10 \sigma)^{2 \alpha+2 \beta+4 \gamma} \varepsilon^{\alpha+2 \beta+2 \gamma} 4 \sigma^{2} \varepsilon\left(1+\frac{1}{2 L} d(X)\right)^{2} \\
& +\sum_{\alpha+\beta+\gamma=1} 72 \frac{\varepsilon_{0}^{1+2 \gamma}}{\delta_{0}^{3+\alpha+2 \beta+4 \gamma}} E_{0} \exp (-\kappa d(X))(10 \sigma)^{2 \alpha+2 \beta+4 \gamma} \varepsilon^{\alpha+2 \beta+2 \gamma} \cdot \sigma^{2} \varepsilon^{2}(2 d L M)^{2} \\
& \left(1+\frac{1}{2 L} d(X)\right)^{2}<\frac{1}{2}\left[\frac{(10 \sigma)^{4} \varepsilon^{2}}{\delta_{0}^{2}}+\frac{(10 \sigma)^{4} \varepsilon^{3}}{\delta_{0}^{3}}+\frac{(10 \sigma)^{6} \varepsilon^{3} \varepsilon_{0}^{2}}{\delta_{0}^{5}}\right] E_{0} \exp (-(\kappa-1) d(X)) \\
& \quad+\left[\frac{(10 \sigma)^{4} \varepsilon^{3} \varepsilon_{0}}{\delta_{0}^{4}}+\frac{(10 \sigma)^{4} \varepsilon^{4} \varepsilon_{0}}{\delta_{0}^{5}}+\frac{(10 \sigma)^{6} \varepsilon^{4} \varepsilon_{0}^{3}}{\delta_{0}^{7}}\right] E_{0} \exp (-(\kappa-1) d(X))(2 d L M)^{2} \\
& <4(10 \sigma)^{4}\left(\frac{\varepsilon}{\varepsilon_{0}}\right)^{2} \frac{1}{\sigma_{1}^{5}}(2 d L M)^{2} E_{0} \exp (-(\kappa-1) d(X)),
\end{aligned}
$$

where we have used the conditions $10 \sigma \varepsilon_{0} \leqq \delta_{0}, \varepsilon \leqq \varepsilon_{0}$, and the equality $\delta_{0}=\sigma_{1} \varepsilon_{0}$. Similarly the second sum can be bounded by

$$
\begin{aligned}
\sum_{\alpha+\beta+\gamma=1} & 12^{2} \frac{\varepsilon_{0}^{2 \gamma}}{\delta_{0}^{2+\alpha+2 \beta+4 \gamma}} E_{0} \exp (-\kappa d(X))(10 \sigma)^{2 \alpha+2 \beta+4 \gamma} \varepsilon^{\alpha+2 \beta+2 \gamma} \\
& \cdot(2 d L M+d M d(X))^{2}(2 \sigma \varepsilon)^{2} \\
< & 18(10 \sigma)^{4}\left(\frac{\varepsilon}{\varepsilon_{0}}\right)^{3} \frac{1}{\sigma_{1}^{4}}(2 d L M)^{2} E_{0} \exp (-(\kappa-1) d(X)) .
\end{aligned}
$$


Finally the last sum can be bounded by

$$
\begin{aligned}
& \frac{1}{2}\left[8 \cdot 6^{2} \frac{1}{\delta_{0}^{2}}\left(4 \sigma^{2} \varepsilon\right)^{2}\left(1+\frac{1}{2 L} d(X)\right)^{4}+2 \cdot 8 \cdot 6^{3} \frac{\varepsilon_{0}}{\delta_{0}^{4}} 4 \sigma^{2} \varepsilon \cdot \sigma^{2} \varepsilon^{2}(2 d L M)^{2}\right. \\
& \left.\cdot\left(1+\frac{1}{2 L} d(X)\right)^{4}+8 \cdot 6^{4} \frac{\varepsilon_{0}^{2}}{\delta_{0}^{6}} \sigma^{4} \cdot \varepsilon^{4}(2 d L M)^{2}\left(1+\frac{1}{2 L} d(X)\right)^{4}\right] \cdot E_{0} \exp (-\kappa d(X)) \\
& <(10 \sigma)^{4}\left(\frac{\varepsilon}{\varepsilon_{0}}\right)^{2} \frac{1}{\sigma_{1}^{6}}(2 d L M)^{2} E_{0} \exp (-(\kappa-1) d(X))
\end{aligned}
$$

There is one additional term, the fourth term on the right-hand side of (3.89), which is irrelevant also. It follows from (3.77), (3.86) that it can be bounded by

$$
\begin{gathered}
\left.2 \cdot 6^{2} \cdot 10 \frac{\sigma^{4} \varepsilon^{2} \varepsilon_{0}}{\delta_{0}^{3}}\left(1+\frac{1}{2 L} d(X)\right)^{2} E_{0} \exp (-\kappa d)(X)\right) \\
<\frac{1}{12}(10 \sigma)^{4}\left(\frac{\varepsilon}{\varepsilon_{0}}\right)^{2} \frac{1}{\sigma_{1}^{3}} E_{0} \exp (-(\kappa-1) d(X))
\end{gathered}
$$

Among the bounds (3.92)-(3.95) the bound (3.94) is the largest one, the others give only small contributions to it. We can bound the four expressions in (3.89) by the right-hand side of (3.94) multiplied by 2 .

Consider the remaining four terms on the right-hand side of (3.89). Let us write them again in the following form:

$$
\begin{aligned}
& -\frac{1}{2}\left\langle v_{1}(y, X ; 1, v, 0),|\psi-\psi(y)|^{2}\right\rangle+\frac{1}{2} v\left\langle v_{2}(y, X ; 1, v, 0),|\psi-\psi(y)|^{2}\right\rangle \\
& +\frac{1}{2}\left\langle v_{0}(y, X ; 1, v, 0),(\psi-\psi(y)) \cdot(\psi-\psi(y))\right\rangle \\
& +\frac{1}{2}\left\langle v_{1}(y, X ; 1, v, 0),|\psi|^{2}-|\psi(y)|^{2}\right\rangle+\left\langle v_{2}(y, X ; 1, v, 0), v h \cdot \psi_{0}-v h \cdot \psi_{0}(y)\right\rangle .
\end{aligned}
$$

In the last two terms the functions $|\psi(x)|^{2}-|\psi(y)|^{2}, v h \cdot \psi_{0}(x)-v h \cdot \psi_{0}(y)$ are antisymmetric functions of $x, y$. We will show that sums over $X$ of the functions $v_{\sigma}(x, y, X ; 1, v, 0)$ are symmetric in $x, y$, so these two terms resummed over $X$ and $y$ in the sum (3.23) will contribute 0. Consider the first three terms in (3.96). We decompose them into sums of simple local expressions and irrelevant expressions by applying a lattice Taylor formula. Such a formula has been used before in a similar context in [2d]. Let us recall it. Let $x, y$ be two points of a unit lattice, and let $\Gamma_{y, x}$ be a unit lattice path from $y$ to $x$. Take the part of this path which is parallel to the unit vector $e_{\mu}$ of the $x_{\mu}$-axis, give it a positive orientation with respect to this vector, and denote by $\left[\Gamma_{y, x}[\mu\right.$ the set of initial points of bonds contained in this part. For a function $f$ defined on the unit lattice we have

$$
\begin{aligned}
f(x) & =f(y)+\sum_{\mu=1}^{d}\left(x_{\mu}-y_{\mu}\right)\left(\partial_{\mu} f\right)(y)+\sum_{\mu=1}^{d} \sum_{x^{\prime} \in\left[\Gamma_{y, x}[\mu\right.} \sum_{b \subset \Gamma_{y, x^{\prime}}} \operatorname{sign}\left(x_{\mu}-y_{\mu}\right)\left(\partial \partial_{\mu} f\right)(b) \\
& =f(y)+(x-y) \cdot(\nabla f)(y)+R_{2}\left(x, y, \partial^{2} f\right) .
\end{aligned}
$$


The remainder $R_{2}$ satisfies the following bound:

$$
\begin{aligned}
\left|R_{2}\left(x, y, \partial^{2} f\right)\right| & \leqq \frac{1}{2}\left(\left|\Gamma_{y, x}\right|+1\right) \mid\left(\Gamma_{y, x} \|\left.\partial^{2} f\right|_{\Gamma_{y, x}}\right. \\
& \leqq \frac{1}{2}(2 d L M+d M d(X))^{2}\left|\partial^{2} f\right|_{X}, \quad x, y \in X,
\end{aligned}
$$

where the last inequality holds, for example, for the path constructed in (3.54). Apply the formula (3.97) to the function $\delta \psi(x)=\psi(x)-\psi(y)$ in the first three terms in (3.96). Each term gives a rise to four terms, for example for the first term we have

$$
\begin{aligned}
\left\langle v_{1}(y, X ; 1, v, 0),|\delta \psi|^{2}\right\rangle= & \left\langle v_{1}(y, X ; 1, v, 0),((x-y) \cdot(\nabla \psi)(y))^{2}\right\rangle \\
& +2\left\langle v_{1}(y, X ; 1, v, 0),((x-y) \cdot(\nabla \psi)(y)) \cdot R_{2}\left(x, y, \partial^{2} \psi\right)\right\rangle \\
& +\left\langle v_{1}(y, X ; 1, v, 0),\left(R_{2}\left(x, y, \partial^{2} \psi\right)\right)^{2}\right\rangle,
\end{aligned}
$$

similarly for the second and third, with some obvious modifications. The last two terms on the right-hand side above are irrelevant, and their analytic extensions can be bounded by

$$
\begin{aligned}
& 12 \frac{1}{\delta_{0}} E_{0} \exp (-\kappa d(X))(2 d L M+d M d(X))^{3} 2 \sigma \varepsilon\left|\partial^{2}\left(\psi+\psi^{\prime}\right)\right|_{X} \\
& \quad+12 \frac{1}{\delta_{0}} E_{0} \exp (-\kappa d(X)) \frac{1}{4}(2 d L M+d M d(X))^{4}\left|\partial^{2}\left(\psi+\psi^{\prime}\right)\right|_{X}^{2} \\
& \leqq \\
& 13 \sigma \frac{\varepsilon}{\varepsilon_{0}}\left|\partial^{2}\left(\psi+\psi^{\prime}\right)\right|_{X} \frac{1}{\sigma_{1}}(2 d L M)^{4} E_{0} \exp (-(\kappa-1) d(X)) .
\end{aligned}
$$

Let us make a few comments on this bound. We apply it to the case $\psi=\psi_{k}^{(j)}$, and then the second order derivatives can be bounded by const. $\sigma^{2-\alpha} \varepsilon$, where $\alpha$ is an arbitrary positive number, the constant depends on $\alpha$. Thus we have a bound by $\sigma^{3-\alpha} \varepsilon^{2}$, which is enough to prove the irrelevance. Unfortunately it is not enough to prove the best possible choice $\gamma_{0}=\min \left\{\frac{d-2}{2}, 1\right\}$, for which we need $\sigma^{4-\alpha} \varepsilon^{2}$. A reason for the worse bound is the second term on the right-hand side of (3.99), in which there is a mixture of first and second order derivatives. Actually such terms should vanish, and we could show it resumming over $X$ and using the symmetries in (H.4). Such an analysis is quite straightforward, but lengthy, and for the sake of brevity we present the above shorter one. We will prove that $\gamma_{0}=\min \left\{\frac{d-2}{2}, \frac{1}{2}\right\}$ is a possible choice, and for $d=3$ it agrees with the best possible one. Consider briefly the second and third terms in (3.96). The corresponding irrelevant terms can be bounded by

$$
8 \cdot 6^{2} \sigma^{2}\left(\frac{\varepsilon}{\varepsilon_{0}}\right)^{2} \frac{1}{\sigma_{1}^{3}}(2 d L M)^{4} E_{0} \exp (-(\kappa-1) d(X)),
$$

where we have used the condition $v \leqq \sigma^{2}$, and by

$$
2 \cdot 12^{2} \sigma \frac{\varepsilon}{\varepsilon_{0}^{2}}\left|\partial^{2}\left(\psi+\psi^{\prime}\right)\right|_{X} \frac{1}{\sigma_{1}^{2}}(2 d L M)^{4} E_{0} \exp (-(\kappa-1) d(X)) .
$$

Finall consider the third term on the right-hand side of (3.51). It is an irrelevant term, and a more careful analysis of this term would be needed only if we wanted to show that we can choose $\gamma_{0}$ in the best possible way. Without doing such an analysis 
we can bound simply its analytic extension using the bounds (3.68), (3.55) by

$$
\begin{aligned}
& \frac{(6 \cdot 3)^{3}}{\delta_{0}^{3}} E_{0} \exp (-\kappa d(X))(2 d L M+d M d(X))^{3}(2 \sigma \varepsilon)^{3} \\
& <36^{3} \sigma^{3}\left(\frac{\varepsilon}{\varepsilon_{0}}\right)^{3} \frac{1}{\sigma_{1}^{3}}(2 d L M)^{3} E_{0} \exp (-(\kappa-1) d(X)) .
\end{aligned}
$$

We have finished this part of the renormalization analysis which can be done for a single term of the second sum in (3.23). Let us summarize the above results in the lemma.

Lemma 3.8. Consider a localized function $\mathscr{E}(y, X ; \psi, v h)$. It has an analytic extension onto the space $\tilde{\Psi}^{c}\left(\delta_{0}, \varepsilon_{0}\right)$ with $\delta_{0}=\delta_{1}=\sigma_{1} \varepsilon_{0}, \varepsilon_{0}=\varepsilon_{j+1}, \sigma_{1}=K_{1}^{-1} \alpha$. We restrict it to a smaller subspace $\Psi^{c}(\delta, \varepsilon)$ with $\sigma, \varepsilon$ satisfying the conditions $\varepsilon \leqq \varepsilon_{0}, \sigma \leqq \sigma_{2}$, where $\sigma_{2}=\left(4 d K_{1} L M\right)^{-1} \alpha$, and we assume that $X$ satisfies the condition (3.59), i.e. $d(X) \leqq \frac{1}{2 d M} \frac{\sigma_{1} \varepsilon_{0}}{\sigma \varepsilon}=\frac{\alpha}{2 d K_{1} M} \frac{\varepsilon_{0}}{\sigma \varepsilon}$. On the space $\Psi(\sigma, \varepsilon)$ we have the representation

$$
\begin{aligned}
\mathscr{E}(y, X ; \psi, v h)-\mathscr{E}(y, X ; \psi(y), v h) \\
=\frac{1}{2}\left\langle v_{1}(y, X ; 1, v, 0), \psi^{2}(x)-\psi^{2}(y)\right\rangle \\
\quad+\left\langle v_{2}(y, X ; 1, v, 0), v h \cdot \psi_{0}(x)-v h \cdot \psi_{0}(y)\right\rangle \\
\quad-\frac{1}{2}\left\langle v_{1}(y, X ; 1, v, 0)-v v_{2}(y, X ; 1, v, 0),\left((x-y) \cdot(\nabla \psi(y))^{2}\right\rangle\right. \\
\quad+\frac{1}{2}\left\langle v_{0}(y, X ; 1, v, 0),\left(\left(x_{1}-y\right) \cdot(\nabla \psi)(y)\right) \cdot\left(\left(x_{2}-y\right) \cdot(\nabla \psi)(y)\right)\right\rangle \\
\quad+\mathscr{E}_{\text {irrel }}(y, X ; \psi, v h),
\end{aligned}
$$

where the last term is a sum of all the irrelevant expressions analyzed above. It has an analytic extension onto the space $\Psi^{c}(\sigma, \varepsilon)$, and the extended function satisfies the inequality

$$
\begin{aligned}
& \left|\mathscr{E}_{\text {irrel }}\left(y, X ; \psi+\psi^{\prime}, v\left(h+h^{\prime}\right)\right)\right|<K_{1} \frac{1}{\alpha}\left(20 d K_{1} L M \frac{1}{\alpha}\right)^{4} \\
& \cdot\left(\sigma^{3}\left(\frac{\varepsilon}{\varepsilon_{0}}\right)^{2}+\sigma \frac{\varepsilon}{\varepsilon_{0}^{2}}\left|\partial^{2}\left(\psi+\psi^{\prime}\right)\right|_{X}\right) E_{0} \exp (-(\kappa-1) d(X)),
\end{aligned}
$$

following from the bounds (3.61),(3.92)-(3.95),(3.100)-(3.103). The functions $v_{1}, v_{2}, v_{0}$ are defined by the formulas (3.73),(3.74),(3.78), i.e. by

$$
\begin{aligned}
v_{1}(x, y, X ; 1, v, 0) & =\left(\frac{\partial}{\partial \psi(x)} \mathscr{E}\right)\left(y, X ; e_{1}, v e_{1}\right) \cdot e_{1}, \\
v_{2}(x, y, X ; 1, v, 0) & =\left(\frac{\partial}{\partial v w} \frac{\partial}{\partial \psi(x)} \mathscr{E}\right)\left(y, X ; e_{1}, v e_{1}\right) \cdot e_{2}, \\
v_{0}\left(x_{1}, x_{2}, y, X ; 1, v, 0\right) & =\operatorname{tr}\left[\left(\frac{\partial^{2}}{\partial \psi\left(x_{1}\right) \partial \psi\left(x_{2}\right)} \mathscr{E}\right)\left(y, X ; e_{1}, v e_{1}\right) \cdot\left(e_{2} \otimes e_{2}\right)\right],
\end{aligned}
$$

and they satisfy the bounds (3.77), (3.80) correspondingly. 
Let us make a few remarks about the above statements. At first let us stress that we have done all the expansions and transformations for functions of real configurations $(\psi, h)$, and then we have constructed analytic extensions of the resulting expressions. This means that the constructed analytic extension of the right-hand side of (3.104) is not necessarily equal to the assumed analytic extension of the left-hand side. The constructions of this subsection may be interpreted as a construction of a mapping from a space of given analytic extensions onto a subspace of new analytic extensions with better bounds. Next, notice that we have not made any effort to find optimal bounds, e.g., the constant in (3.105) is just a simplest common constant for all terms in the expression.

Eventually we apply the representation (3.104) to configurations $\psi=\psi_{k}^{(j)}$, where the function $\psi_{k}^{(j)}$ is considered on the space $\Xi_{k}^{c}\left(1, \varepsilon_{k}\right)$. The values of the function are in the space $\Psi_{j}^{c}\left(C_{0} L^{j} \eta, \varepsilon_{k}\right)$ with $C_{0} \geqq 3$, and the constants $\sigma=C_{0} L^{j} \eta$, $\varepsilon=\varepsilon_{k}$ satisfy the conditions of the lemma if $k-j \geqq n_{1}$. The function $\psi_{k}^{(j)}$ satisfies also the bound

$$
\left|\partial^{2} \psi_{k}^{(j)}\right|<K_{1} C_{\alpha_{1}}\left(L^{j} \eta\right)^{2-\alpha_{1}} \varepsilon_{k}
$$

for any constant $\alpha_{1}, 0<\alpha_{1} \leqq 1$, where $C_{\alpha_{1}}$ is an absolute constant depending on $\alpha_{1}$ only. It follows from the formula (2.14), the inclusions in (3.22), and properties of $\phi_{k}$ discussed in the next paper. Thus the bound (3.105) in this case yields the bound

$$
\begin{aligned}
&\left|\mathscr{E}_{\text {irrel }}\left(y, X ; \psi_{k}^{(j)}, v\left(h+h^{\prime}\right)\right)\right|< K_{1}^{2} C_{\alpha_{1}} \frac{1}{\alpha}\left(20 d C_{0} K_{1} L^{1+\frac{\alpha}{2}} M \frac{1}{\alpha}\right)^{4} \\
& \cdot\left(L^{j} \eta\right)^{d+\alpha_{s}} E_{0} \exp (-(\kappa-1) d(X)),
\end{aligned}
$$

where $\alpha_{3}=1-2 \gamma-\alpha_{1}=2\left(\frac{1}{2}-\gamma\right)-\alpha_{1} \geqq 2\left(\gamma_{0}-\gamma\right)-\alpha_{1}$ and we have taken now $\gamma_{0}=\min \left\{\frac{d-2}{2}, \frac{1}{2}\right\}$. We assume $\gamma<\gamma_{0}$, hence $\gamma_{0}-\gamma>0$ and we may take $\alpha_{1}=\gamma_{0}-\gamma$, we have then $\alpha_{3} \geqq \gamma_{0}-\gamma>0$. Notice also that $\alpha=\frac{d-2}{2}-\gamma \geqq \gamma_{0}-\gamma$, and that $\gamma_{0}=\frac{1}{2}$ for $d \geqq 3$.

To analyze further the first four terms on the right-hand side of (3.104) we have to resum the representation over $X$ and $y$. Denote $\rho=\sigma \frac{\varepsilon}{\varepsilon_{0}}$ and $d(\rho)=\frac{\alpha}{2 d K_{1} M} \frac{1}{\rho}$. We write

$$
\begin{aligned}
\sum_{y \in T^{(j)}} & \sum_{X \in \mathscr{D}_{j} ; y \in X}(\mathscr{E}(y, X ; \psi, v h)-\mathscr{E}(y, X ; \psi(y), v h)) \\
= & \sum_{y \in T^{(\prime)}} \sum_{X \in \mathscr{Q}_{;} ; y \in X, d(X) \leqq d(\rho)} \mathscr{E}_{i r r e l}(y, X ; \psi, v h) \\
& +\sum_{y \in T^{(j)}} \sum_{X \in \mathscr{D}_{j} ; y \in X, d(X)>d(\rho)}[\mathscr{E}(y, X ; \psi, v h)-\mathscr{E}(y, X ; \psi(y), v h) \\
& -\frac{1}{2}\left\langle v_{1}(y, X ; 1, v, 0), \psi^{2}(x)-\psi^{2}(y)\right\rangle \\
& \left.-\left\langle v_{2}(y, X ; 1, v, 0) ; v h \cdot \psi_{0}(x)-v h \cdot \psi_{0}(y)\right\rangle\right] \\
& +\sum_{x, y \in T^{(\omega)}}\left[\frac{1}{2} v_{1}(x, y ; 1, v, 0)\left(\psi^{2}(x)-\psi^{2}(y)\right)\right. \\
& \left.+v_{2}(x, y ; 1, v, 0)\left(v h \cdot \psi_{0}(x)-v h \cdot \psi_{0}(y)\right)\right]
\end{aligned}
$$




$$
\begin{aligned}
& +\sum_{y \in T^{(j)}} \frac{1}{2} \sum_{\mu, v=1}^{d}\left(v_{0, \mu, v}^{(\infty)}-v_{1, \mu, v}^{(\infty)}+v v_{2, \mu, v}^{(\infty)}\right)\left(\partial_{\mu} \psi\right)(y) \cdot\left(\partial_{v} \psi\right)(y) \\
& -\sum_{y \in T^{(j)}} \frac{1}{2} \sum_{\mu, v=1}^{d}\left(v_{0, \mu, v}^{(\rho)}(y)-v_{1, \mu, v}^{(\rho)}(y)+v v_{2, \mu, v}^{(\rho)}(y)\right)\left(\partial_{\mu} \psi\right)(y) \cdot\left(\partial_{v} \psi\right)(y),
\end{aligned}
$$

where the functions $v_{\sigma}(x, y ; 1, v, 0), \sigma=1,2, v_{0}\left(x_{1}, x_{2}, y ; 1, v_{0}\right)$ are sums over all $X \in \mathscr{D}_{j}=\mathscr{D}_{j}\left(T_{\xi}\right)$ of the corresponding localized functions, and are given by the formulas (3.106), in which the function $\mathscr{E}(y, X ; \psi, v h)$ is replaced by the sum over all $X$, which is equal to the function $\mathscr{E}(y ; \psi, v h)$. Functions $v_{\sigma}^{(\infty)}$ are defined as thermodynamic limits of the corresponding $v_{\sigma}$ as $T_{\xi} \rightarrow \xi \mathbf{Z}^{d}$, i.e.

$$
\begin{aligned}
v_{\sigma}^{(\infty)}(x, y) & =\lim _{T_{\xi} \rightarrow \xi \mathbf{Z}^{d}} v_{\sigma}(x, y ; 1, v, 0)=\sum_{X \in \mathscr{Z}_{j}\left(\xi z^{d}\right) \cdot y \in X} v_{\sigma}(x, y, X ; 1, v, 0), \\
v_{0}^{(\infty)}\left(x_{1}, x_{2}, y\right) & =\lim _{T_{\xi} \rightarrow \xi \mathbf{Z}^{d}} v_{0}\left(x_{1}, x_{2}, y ; 1, v, 0\right) \\
& =\sum_{X \in \mathscr{D}_{(}\left(\xi \mathbf{Z}^{d}\right): y \in X} v_{0}\left(x_{1}, x_{2}, y, X ; 1, v, 0\right) .
\end{aligned}
$$

Functions $v_{\sigma}^{(\rho)}(x, y), \sigma=1,2, v_{0}^{(\rho)}\left(x_{1} x_{2}, y\right)$ are given by the corresponding sums on the right-hand sides above, in which the summations over $X$ are restricted by the condition $d(X)>d(\rho)$. Finally the constants $v_{\sigma, \mu, v}^{(\infty)}, v_{0, \mu, v}^{(\infty)}$ are given by the formulas

$$
\begin{gathered}
v_{\sigma ; \mu, v}^{(\infty)}=\sum_{x \in \mathbf{Z}^{d}} v_{\sigma}^{(\infty)}(x, y)\left(x_{\mu}-y_{\mu}\right)\left(x_{v}-y_{v}\right), \\
v_{0 ; \mu, v}^{(\infty)}=\sum_{x_{1}, x_{2} \in \mathbf{Z}^{d}} v_{0}^{(\infty)}\left(x_{1}, x_{2}, y\right)\left(x_{1, \mu}-y_{\mu}\right)\left(x_{2, v}-y_{v}\right) .
\end{gathered}
$$

Similarly the functions $v_{\sigma, \mu, v}^{(\infty)}(y), v_{0 ; \mu, v}^{(\infty)}(y)$ are given by the above formulas, but with functions $v_{\sigma}^{(\infty)}(x, y), v_{0}^{(\infty)}\left(x_{1}, x_{2}, y\right)$ replaced by $v_{\sigma}^{(\rho)}(x, y), v_{0}^{(\rho)}\left(x_{1}, x_{2}, y\right)$. It is clear that the sums in the above definitions are convergent, and do not depend on $y$ in (3.111), by translation invariance. Let us analyze more closely symmetry properties and bounds for the introduced functions and constants. We assume that the functions $\mathscr{E}(y ; \psi, v h)$ satisfies the basic Euclidean symmetry (H.4), or (2.23). This symmetry and the above definitions imply

$$
v_{\sigma}(r x, r y)=v_{\sigma}(x, y), \quad \sigma=1,2, v_{0}\left(r x_{1}, r x_{2}, r y\right)=v_{0}\left(x_{1}, x_{2}, y\right)
$$

for all Euclidean transformations $r$ of the lattice $T^{(j)}$. The same holds for the corresponding thermodynamic limits. In particular applying translations and a reflection at the origin we conclude that the functions $v_{\sigma}(x, y)$ are symmetric in $x, y$ :

$$
v_{\sigma}(x, y)=v_{\sigma}(x-y, 0)=v_{\sigma}(-(x-y), 0)=v_{\sigma}(y, x) .
$$

This implies that the third sum on the right-hand side of (3.109) is equal to 0 , because we sum over all $x, y \in T^{(j)}$ the products of symmetric and antisymmetric functions, so we sum over $x, y$ an antisymmetric function in $x, y$. The symmetries (3.112) for the thermodynamic limits of the functions imply that the matrices defined by the elements $v_{1 ; \mu, v}^{(\infty)}, v_{0 ; \mu, v}^{(\infty)}$ are proportional to the identity matrix. Thus we 
can write the fourth sum in (3.109) in the following way:

$$
\frac{1}{2}\left(v_{0}^{(\infty)}-v_{1}^{(\infty)}+v v_{2}^{(\infty)}\right) \sum_{y \in T^{(j)}} \sum_{\mu=1}^{d}\left|\left(\partial_{\mu} \psi\right)(y)\right|^{2}=\frac{1}{2}\left(v_{0}^{(\infty)}-v_{1}^{(\infty)}+v v_{2}^{(\infty)}\right)\|\partial \psi\|^{2},
$$

where

$$
v_{\sigma}^{(\infty)}=v_{\sigma, \mu, \mu}^{(\infty)}=\frac{1}{d} \sum_{\mu=1}^{d} v_{\sigma ; \mu, \mu}^{(\infty)}, \quad \sigma=0,1,2 .
$$

The last sum in (3.109) remains unchanged. Let us introduce the following definition:

$$
\begin{aligned}
\mathscr{E}_{(\sigma, \varepsilon)-\text { irrel }}(y, X ; \psi, v h)= & \mathscr{E}_{\text {irrel }}(y, X ; \psi, v h), \quad \text { if } d(X) \leqq d(\rho), \rho=\sigma \frac{\varepsilon}{\varepsilon_{0}}, \\
\mathscr{E}_{(\sigma, \varepsilon)-\text { irrel }}(y, X ; \psi, v h)= & \mathscr{E}(y, X ; \psi, v h)-\mathscr{E}(y, X ; \psi(y), v h) \\
& -\frac{1}{2}\left\langle v_{1}(y, X ; 1, v, 0), \psi^{2}-\psi^{2}(y)\right\rangle \\
& -\left\langle v_{2}(y, X ; 1, v, 0), v h \cdot \psi_{0}-v h \cdot \psi_{0}(y)\right\rangle,
\end{aligned}
$$

if $d(X)>d(\rho)$. The functions above have analytic extensions onto the spaces $\Psi^{c}(\sigma, \varepsilon)$ with $\sigma, \varepsilon$ satisfying the conditions in Lemma 3.8. Actually in the second case, i.e. for $d(X)>d(\rho)$, they are analytic on the larger space $\widetilde{\Psi}^{c}\left(\delta_{0}, \varepsilon_{0}\right)$. Bounds for the functions have been written in the first case in (3.105). In the second case they have been partially written in (3.66) for the special cases $\tilde{\Psi}_{j}^{c}\left(C_{0} L^{j} \eta, \varepsilon_{k}\right)$. On the space $\Psi^{c}(\sigma, \varepsilon)$ they can be bounded by

$$
\begin{gathered}
2 E_{0} \exp (-\kappa d(X))+24 \sigma^{2} \frac{\varepsilon}{\delta_{0}} E_{0} \exp (-\kappa d(X))+6^{2}(10 \sigma)^{2} \frac{\varepsilon^{2} \varepsilon_{0}}{\delta_{0}^{3}} E_{0} \exp (-\kappa d(X)) \\
<\left(2 d K_{1} M \frac{1}{\alpha}\right)^{4} \sigma^{3}\left(\frac{\varepsilon}{\varepsilon_{0}}\right)^{3} E_{0} \exp (-(\kappa-1) d(X))
\end{gathered}
$$

hence they certainly satisfy the bound (3.105). This bound is universal for the two cases.

Finally we want to obtain bounds for the functions $v_{\sigma ; \mu, v}^{(\rho)}(y)$. We have

$$
v_{\sigma ; \mu, v}^{(\rho)}(y)=\sum_{X \in \mathscr{D},\left(\xi \mathbf{Z}^{d}\right): y \in X, d(X)>d(\rho)}\left\langle v_{\sigma}(y, X ; 1, v, 0),\left(x_{\mu}-y_{\mu}\right)\left(x_{v}-y_{v}\right)\right\rangle,
$$

hence

$$
\begin{aligned}
\left|v_{1 ; \mu, v}^{(\rho)}(y)\right| & <12 \frac{1}{\delta_{0}} E_{0} \sum_{X \in \mathscr{Q},\left(\xi \mathbf{Z}^{d}\right): y \in X, d(X)>d(\rho)} \exp (-\kappa d(X))(2 d L M+d M d(X))^{2} \\
& <12(2 d L M)^{2} \frac{1}{\delta_{0}} E_{0} e^{-d(\rho)} \sum_{X \in \mathscr{D},\left(\xi \mathbf{Z}^{d}\right): y \in X} \exp (-(\kappa-2) d(X)) \\
& \leqq 12 K_{0} E_{0}\left(2 d K_{1} L M \frac{1}{\alpha}\right)^{3} \sigma \frac{\varepsilon}{\varepsilon_{0}^{2}}
\end{aligned}
$$

Obviously we can obtain a bound by any power of $\rho$, but we need only the power 1 . 
In the same way we obtain bounds for $v_{2 ; \mu, v}^{(\rho)}(y)$ and $v_{0 ; \mu, v}^{(\rho)}(y)$, for example

$$
\begin{aligned}
& \left|v_{2, \mu, v}^{(\rho)}(y)\right|<\frac{2}{M} K_{0} E_{0}\left(2 d K_{1} L M \frac{1}{\alpha}\right)^{4} \sigma \frac{\varepsilon}{\varepsilon_{0}^{3}}, \\
& \left|v_{0 ; \mu, v}^{(\rho)}(y)\right|<8 \cdot 12 K_{0} E_{0}\left(2 d K_{1} L M \frac{1}{\alpha}\right)^{3} \sigma \frac{\varepsilon}{\varepsilon_{0}^{3}} .
\end{aligned}
$$

Combining the above bounds together we obtain

$$
\left|v_{0, \mu, v}^{(\rho)}(y)-v_{1 ; \mu, v}^{(\rho)}(y)+v v_{2 ; \mu, v}^{(\rho)}(y)\right|<K_{0} E_{0}\left(2 d K_{1} L M \frac{1}{\alpha}\right)^{4} \frac{1}{\varepsilon_{0}^{2}} .
$$

Notice also that the same arguments yield

$$
\left|v_{0}^{(\infty)}-v_{1}^{(\infty)}+v v_{2}^{(\infty)}\right|<K_{0} E_{0}\left(2 d K_{1} L M \frac{1}{\alpha}\right)^{3} \sigma \frac{\varepsilon}{\varepsilon_{0}^{2}} .
$$

The constant estimated above determines a renormalization counterterm in the future analysis of renormalization group equations, and the bound gives a magnitude of this counterterm.

We summarize again the results of the above analysis in the following lemma.

Lemma 3.9. Consider a family of functions $\mathscr{E}(y, X ; \phi, v h)$ satisfying the inductive assumptions (H.3), (H.4), (H.5). They have analytic extensions onto the spaces $\Psi^{c}(\sigma, \varepsilon)$ described in Lemma 3.8, and on the space $\Psi(\sigma, \varepsilon)$ we have the representation

$$
\begin{aligned}
& \sum_{y \in T^{(j)}} \sum_{X \in \mathscr{D}_{j}: y \in X}[\mathscr{E}(y, X ; \psi, v h)-\mathscr{E}(y, X ; \psi(y), v h)] \\
& =\frac{1}{2}\left(v_{0}^{(\infty)}-v_{1}^{(\infty)}+v v_{2}^{(\infty)}\right)\|\partial \psi\|^{2}-\frac{1}{2}\left\langle\partial \psi,\left(v_{0}^{(\rho)}-v_{1}^{(\rho)}+v v_{2}^{(\rho)}\right) \partial \psi\right\rangle \\
& \quad+\sum_{y \in T^{(j)}} \sum_{X \in \mathscr{D}_{j}: y \in X} \mathscr{E}_{(\sigma, \varepsilon)-\text { irrel }}(y, X ; \psi, v h) .
\end{aligned}
$$

The functions $\mathscr{E}_{(\sigma, \varepsilon)-i r r e l}(y, X ; \psi, v h)$ have analytic extensions onto the space $\Psi^{c}(\sigma, \varepsilon)$, and they satisfy the bound (3.105). The first two terms on the right-hand side are sums of local expressions given correspondingly by (3.114) and the last sum in (3.109). A term of this sum for a fixed point $y$ can be bounded by $2 d^{2} K_{0} E_{0}\left(2 d K_{1} L M \frac{1}{\alpha}\right)^{4} \sigma^{3}\left(\frac{\varepsilon}{\varepsilon_{0}}\right)^{3}$, hence it is an irrelevant quantity.

Thus only the first term on the right-hand side of (3.120) is not irrelevant, it is proportional to the gradient term in the main action. If we assume that the coefficient in front of this term is equal to 0 , then the whole expression in (3.120) will be irrelevant. This leads to the inductive hypothesis (H.7). The function $\mathscr{E}^{(j)}\left(\psi_{j}, v_{j} h\right)$ defining the $\mathrm{j}^{\text {th }}$ term of the effective action has the localization expansion (H.2),(H.5) which obviously satisfies the assumptions of the above lemma, therefore it has the representation (3.120) for every $(\sigma, \varepsilon)$ satisfying the conditions of the lemma. In this representation the coefficient in the first term on the right-hand side does not depend on $\sigma, \varepsilon$, and is given by the corresponding formulas (3.110), (3.111), (3.115). Denoting the constants by $v_{\sigma}^{(j, \infty)}$ we write them in terms of derivatives of the function $\mathscr{E}^{(j)}\left(y ; \psi, v_{j} h\right)$ in the thermodynamic limit. They are 
expressed in terms of such derivatives by the formulas (3.106), (3.110), (3.111), (3.115). In the formula for $v_{0}^{(j, \infty)}$ we apply the identity

$$
\left(x_{1}-y\right) \cdot\left(x_{2}-y\right)=-\frac{1}{2}\left|x_{1}-x_{2}\right|^{2}+\frac{1}{2}\left|x_{1}-y\right|^{2}+\frac{1}{2}\left|x_{2}-y\right|^{2},
$$

where now $|x|$ denotes the Euclidean norm of the point $x$. We obtain

$$
\begin{aligned}
v_{0}^{(j, \infty)} & -v_{1}^{(j, \infty)}+v_{j} v_{2}^{(j, \infty)} \\
= & -\frac{1}{2 d} \sum_{x, x^{\prime} \in \mathbf{Z}^{d}} \operatorname{tr}\left[\left(\frac{\partial^{2}}{\partial \psi(x) \partial \psi\left(x^{\prime}\right)} \mathscr{E}^{\mathscr{E}(j)}\right)\left(y ; e_{1}, v_{j} e_{1}\right) \cdot\left(e_{2} \otimes e_{2}\right)\right]\left|x-x^{\prime}\right|^{2} \\
& +\frac{1}{d}\left\{\sum_{x, x^{\prime} \in \mathbf{Z}^{d}} \operatorname{tr}\left[\left(\frac{\partial^{2}}{\partial \psi(x) \partial \psi\left(x^{\prime}\right)} \mathscr{E}^{(j)}\right)\left(y ; e_{1}, v_{j} e_{1}\right) \cdot\left(e_{2} \otimes e_{2}\right)\right]|x-y|^{2}\right. \\
& -\sum_{x \in \mathbf{z}^{d}}\left(\frac{\partial}{\partial \psi(x)} \mathscr{E}^{(j)}\right)\left(y ; e_{1}, v_{j} e_{1}\right) \cdot e_{2}|x-y|^{2} \\
& \left.+v_{j} \sum_{x \in \mathbf{z}^{d}}\left(\frac{\partial}{\partial\left(v_{j} w\right)} \frac{\partial}{\partial \psi(x)} \mathscr{E}^{(j)}\right)\left(y ; e_{1}, v_{j} e_{1}\right) \cdot e_{2}|x-y|^{2}\right\}
\end{aligned}
$$

For the second term above we have used the symmetry in the variables $x, x^{\prime}$. The expression above can be simplified essentially, in fact we will prove that the expression in the curly brackets is equal to 0 . Consider again more generally a function $\mathscr{E}(\psi, v h)$ which is defined and analytic on some complex neighborhood of the constant configurations $e_{1}, v e_{1}$. The function may be defined on configurations on the whole lattice $\mathbf{Z}^{d}$, or restricted to some subset $X$. Assume that the function is invariant with respect to the orthogonal group, i.e. we have $\mathscr{E}(R \psi, v R h)=\mathscr{E}(\psi, v h)$ for $R \in O(N)$. Take a one-parameter subgroup $\exp \lambda A, A$ is an antisymmetric matrix, and differentiate the identity with respect to $\lambda$ at $\lambda=0$. This yields

$$
\left\langle\left(\frac{\partial}{\partial \psi} \mathscr{E}\right)(\psi, v h), A \psi\right\rangle+\left(\frac{\partial}{\partial(v h)} \mathscr{E}\right)(\psi, v h) \cdot v A h=0 .
$$

This is a basic identity, the Ward-Takahashi identity for the global $O(N)$-symmetry. We can obtain a whole sequence of identities differentiating the above one. One differentiation with respect to $\psi(x)$ yields

$$
\begin{aligned}
& \sum_{x^{\prime}}\left(\frac{\partial^{2}}{\partial \psi(x) \partial \psi\left(x^{\prime}\right)} \mathscr{E}\right)(\psi, v h) \cdot A \psi\left(x^{\prime}\right)-A\left(\frac{\partial}{\partial \psi(x)} \mathscr{E}\right)(\psi, v h) \\
& \quad+\left(\frac{\partial}{\partial(v h)} \frac{\partial}{\partial \psi(x)} \mathscr{E}\right)(\psi, v h) \cdot v A h .
\end{aligned}
$$

Take the constant configuration $\psi=e_{1}, h=e_{1}$, and anti-symmetric matrix $A$ with the only non-zero elements $A_{2,1}=-A_{1,2}=1$, then the above identity yields

$$
\begin{aligned}
& \sum_{x^{\prime}}\left(\frac{\partial^{2}}{\partial \psi_{2}(x) \partial \psi_{2}\left(x^{\prime}\right)} \mathscr{E}\right)\left(e_{1}, v e_{1}\right)-\left(\frac{\partial}{\partial \psi_{1}(x)} \mathscr{E}\right)\left(e_{1} ; v e_{1}\right) \\
& \quad+v\left(\frac{\partial}{\partial\left(v h_{2}\right)} \frac{\partial}{\partial \psi_{2}(x)} \mathscr{E}\right)\left(e_{1}, v e_{1}\right)=0 .
\end{aligned}
$$


We apply it to the function $\mathscr{E}^{(j)}\left(y ; \psi, v_{j} h\right)$ in the thermodynamic limit, and we obtain an identity which implies immediately that the expression in the curly brackets in (3.121) is equal to 0 . Thus in the formula (3.121) only the first sum on the right-hand side remains. We transform it further using the translation invariance. The variables $x, x^{\prime}, y$ are replaced by $x-x^{\prime}, 0, y-x^{\prime}$, then the summation over $x$ allows us to write $x$ instead of $x-x^{\prime}$, and the summation over $x^{\prime}$ can be replaced by a summation over $y$, and $y-x^{\prime}$ can be replaced by $y$. The summation over $y$ can be applied directly to the functions $\mathscr{E}^{(j)}(y)$, and it yields the whole effective action $\mathscr{E}^{(j)}$. More precisely we have

$$
\begin{aligned}
v_{0}^{(j, \infty)} & -v_{1}^{(j, \infty)}+v_{j} v_{2}^{(j, \infty)} \\
= & -\frac{1}{2 d} \sum_{x \in \mathbf{Z}^{d}} \operatorname{tr}\left[\left(\frac{\partial^{2}}{\partial \psi(x) \partial \psi(0)} \mathscr{E}^{(j)}\right)\left(y ; e_{1}, v_{j} e_{1}\right) \cdot\left(e_{2} \otimes e_{2}\right)\right]|x|^{2} \\
= & -\frac{1}{2 d} \sum_{x \in \mathbf{Z}^{d}} \operatorname{tr}\left[\lim _{T_{\xi} \rightarrow \zeta \mathbf{Z}^{d}}\left(\frac{\partial^{2}}{\partial \psi(x) \partial \psi(0)} \mathscr{E}^{(j)}\right)\left(e_{1}, v_{j} e_{1}\right) \cdot\left(e_{2} \otimes e_{2}\right)\right]|x|^{2} \\
= & -\frac{1}{2 d} \sum_{x \in \mathbf{Z}^{d}} v^{(j, \infty)}(x)|x|^{2}=\frac{1}{2 d}\left(\Delta_{p} v^{(j, \infty)}\right)(0) \\
= & \frac{1}{2}\left(\frac{\partial^{2}}{\partial p_{\mu}^{2}} v^{(j, \infty)}\right)(0)=0,
\end{aligned}
$$

by $(\mathrm{H} .7)$, where $v^{(j, \infty)}(p)=\sum_{x \in \mathbf{Z}^{d}} e^{-i p \cdot x} v^{(j, \infty)}(x)$ is the Fourier transform of the function $v^{(j, \infty)}$. This is the most convenient formulation of the inductive hypothesis, in particular the last expression is a basis of calculations in the future.

Finally we combine together all the results obtained in this subsection in the following proposition.

Proposition 3.10. Consider an effective action $\mathscr{E}^{(j)}\left(\psi, v_{j} h\right)$ satisfying the inductive assumptions (H.2)-(H.7). For any $\sigma, \varepsilon$ satisfying the conditions $\varepsilon \leqq \varepsilon_{0}=\varepsilon_{j+1}$, $\sigma \leqq \sigma_{2}=\left(4 d K_{1} L M\right)^{-1} \alpha$ it has the representation

$$
\begin{aligned}
\mathscr{E}^{(j)}\left(\psi, v_{j} h\right)= & \sum_{y \in T^{(j)}} \mathscr{V}^{(j)}\left(\psi(y), v_{j} h\right)-\frac{1}{2}\left\langle\partial \psi,\left(v_{0}^{(j, \rho)}-v_{1}^{(j, \rho)}+v_{j} v_{2}^{(j, \rho)}\right) \partial \psi\right\rangle \\
& +\sum_{y \in T^{(j)}} \sum_{X \in \mathscr{D}_{j}: y \in X} \mathscr{E}_{(\sigma, \varepsilon)-\text { irrel }}^{(j)}\left(y, X ; \psi, v_{j} h\right)
\end{aligned}
$$

holding on the space $\Psi_{j}(\sigma, \varepsilon)$. The functions in this representation have analytic extensions onto the space $\Psi_{j}^{c}(\sigma, \varepsilon)$, and the extended functions satisfy the bounds (3.47), (3.118) and (3.105) correspondingly.

Let us make a few comments on the above proposition. Notice that now all the terms on the right-hand side of (3.126) are irrelevant, and there is no need to distinguish between them. The first two terms are sums over $y \in T^{(j)}$ of local expressions. For each $y$ we take the cube $\square$ from the cover $\pi_{j}$ which contains the point $y$ in the central large cube of $\square$, and we assign the local expression to $\mathscr{E}_{(\sigma, \varepsilon)-i r r e l}^{(j)}\left(y, \square ; \psi, v_{j} h\right)$. We obtain the representation (3.126) with only the last sum on the right-hand side. Terms of this sum are modified for the corresponding $X=\square$, but it follows from the bounds (3.47), (3.118) that they satisfy the bound 
(3.105) with an additional factor $K_{0}$. For the future reference let us write the representation explicitly:

$$
\mathscr{E}^{(j)}\left(\psi, v_{j} h\right)=\sum_{y \in T^{(j)}} \sum_{X \in \mathscr{D}_{j}: y \in X} \mathscr{E}_{(\sigma, \varepsilon)-i r r e l}^{(j)}\left(y, X ; \psi, v_{j} h\right)
$$

on $\Psi_{j}(\sigma, \varepsilon)$, and the functions on the right-hand side have analytic extensions onto the space $\Psi_{j}^{c}(\sigma, \varepsilon)$. Notice again that this defines an analytic extension of effective action on the left-hand side, which may be different from the originally assumed extension.

Consider the effective action on configurations $\psi=\psi_{k}^{(j)}$. By Lemma 3.2 the function $\psi_{k}^{(j)}$ maps the space $\Xi_{k}^{c}\left(1, \varepsilon_{k}\right)$ into $\Psi_{j}^{c}\left(C_{0} L^{j} \eta, \varepsilon_{k}\right)$ with $C_{0} \geqq 3$. The last space satisfies the conditions of Proposition 3.10 if $C_{0} L^{j} \eta \frac{\varepsilon_{k}}{\varepsilon_{j+1}} \leqq \sigma_{2}$, i.e. if $k-j \geqq n_{1}$. Thus we can formulate the following conclusions.

Corollary 3.11. Let the effective actions $\mathscr{E}^{(j)}\left(\psi, v_{j} h\right)$ satisfy the assumptions of Proposition 3.10. Then for $\sigma, \varepsilon$ as in this proposition they have the representations (3.127) with the terms which are analytic on $\Psi_{j}^{c}(\sigma, \varepsilon)$ and satisfy the bound (3.105) with an additional factor $K_{0}$. Substituting the function $\psi_{k}^{(j)}$ instead of $\psi$ we obtain that the terms of (3.127) are analytic $\Xi_{k}^{c}\left(1, \varepsilon_{k}\right)$ for $k-j \geqq n_{1}$, and they satisfy the bound

$$
\begin{gathered}
\left|\mathscr{E}_{\left(C_{0} L^{\prime} \eta, \varepsilon_{k}\right)-r r r e l}^{(j)}\left(y, X ; \psi_{k}^{(j)}, v_{j}\left(h+h^{\prime}\right)\right)\right|<K_{\alpha, \alpha_{1}}\left(L^{j} \eta\right)^{d+\alpha_{3}} . \\
\cdot E_{0} \exp \left(-(\kappa-1) d_{j}(X)\right),
\end{gathered}
$$

where $\alpha_{3}=2\left(\gamma_{0}-\gamma\right)-\alpha_{1}$, and

$$
K_{\alpha, \alpha_{1}}=K_{0} K_{1}^{2} C_{\alpha_{1}} \frac{1}{\alpha}\left(20 d C_{0} K_{1} L^{1+\frac{\alpha}{2}} M \frac{1}{\alpha}\right)^{4} .
$$

This bound and the representation (3.127) imply

$$
\begin{aligned}
& \left|\mathscr{E}^{(j)}\left(\psi_{k}^{(j)}\left(\psi+\psi^{\prime}, v_{k}\left(h+h^{\prime}\right)\right), v_{j}\left(h+h^{\prime}\right)\right)\right|<K_{\alpha, \alpha_{1}}\left(L^{j} \eta\right)^{d+\alpha_{3}} E_{0} K_{0}\left|T_{1}^{(j)}\right| \text { (3.130) } \\
\left|\mathscr{E}_{k}\left(\psi+\psi^{\prime}, h+h^{\prime}\right)\right|<\sum_{j=1}^{k-n_{1}} K_{\alpha, \alpha_{1}}\left(L^{j} \eta\right)^{d+\alpha_{3}} E_{0} K_{0}\left|T_{1}^{(j)}\right| & +\sum_{j=k-n_{1}+1}^{k} E_{0} K_{0}\left|T_{1}^{(j)}\right|<K_{\alpha, \alpha_{1}} E_{0} K_{0}\left|T_{1}^{(k)}\right| \frac{L^{-n_{1} \alpha_{3}}}{1-L^{-\alpha_{3}}}+E_{0} K_{0}\left|T_{1}^{(k)}\right| \frac{L^{d n_{1}}-1}{L^{d}-1} \\
& \quad\left(K_{0} \frac{1}{\alpha_{3}} K_{\alpha, \alpha_{1}}+2 K_{0}\left(4 d C_{0} K_{1} L^{1+\alpha} M \frac{1}{\alpha}\right)^{d}\right) E_{0}\left|T_{1}^{(k)}\right|,
\end{aligned}
$$

where the last inequality follows from the definition of $n_{1}$.

The bound (3.131) is the fundamental uniform bound for the effective actions $\mathscr{E}_{k}$, i.e. uniform in the rescaled volume $\left|T_{1}^{(k)}\right|$. It is a basis of the renormalization group method, and the reason for the renormalization procedure and conditions.

Acknowledgements. The author would like to express his gratitude to Professors M. O'Caroll and R. Schor for their hospitality, encouragement and many useful discussions connected with this work, to Professor T. Spencer for many discussions and enlightening comments, to Professor J. Fröhlich for his hospitality and invitation to E.T.H. in Zürich, where part of the work has been done, and to Professor D. Brydges for his suggestions leading to a new organization of these papers. 


\section{References}

1. Balaban, T., Imbrie, J., Jaffe, A.: Commun. Math. Phys. (a) 97, 299 (1985); (b) 114, 257 (1988)

2. Balaban, T.: Commun. Math. Phys. (a) 85, 603 (1982); (b) 86, 555 (1982); (c) 89, 571 (1983); (d) 109, 249 (1987); (e) 116, 1 (1988)

3. Bleher, P.M., Major, P.: (a) Commun. Math. Phys. 125, 43 (1989); (b) Ann. Inst. Henri Poincaré, Phys. Theor. 49, 1 (1988)

4. Glimm, J., Jaffe, A., Spencer, T.: Ann. Phys. 101, 610, 631 (1976)

5. Fröhlich, J., Simon, B., Spencer, T.: Commun. Math. Phys. 50, 79 (1976)

6. Fröhlich, J., Pfister, C.-E.: Commun. Math. Phys. 89, 303 (1983)

7. Negele, J.W., Orland, H.: Quantum Many-Particle Systems. Reading MA: Addison-Wesley (1988)

8. Schor, R., O'Carroll, M.: Common. Math. Phys. 138, 487 (1991)

9. Zinn-Justin, J.: Quantum Field Theory and Critical Phenomena. Oxford: Oxford Science Publication, 1989

Communicated by D. Brydges 